Accepted in ApJ

\title{
New isolated planetary mass objects and the stellar and substellar mass function of the $\sigma$ Orionis cluster
}

\author{
K. Peña Ramírez ${ }^{1}$, V. J.S. Béjar ${ }^{1}$ \\ Instituto de Astrofísica de Canarias, C/. Vía Láctea s/n, E-38205 La Laguna, Tenerife, Spain \\ karla@iac.es, vbejar@iac.es \\ M. R. Zapatero Osorio \\ Centro de Astrobiología (CSIC-INTA), Crta. Ajalvir km 4, E-28850 Torrejón de Ardoz, Madrid, \\ Spain \\ mosorio@cab.inta-csic.es \\ M. G. Petr-Gotzens \\ European Southern Observatory, Karl-Schwarzschild-Str. 2, 85748, Garching bei München, \\ Germany \\ mpetr@eso.org \\ and \\ E. L. Martín \\ Centro de Astrobiología (CSIC-INTA), Crta. Ajalvir km 4, E-28850 Torrejón de Ardoz, Madrid, \\ Spain \\ ege@cab.inta-csic.es
}

\begin{abstract}
We report on our analysis of the VISTA Orion $Z Y J H K_{s}$ photometric data (completeness magnitudes of $Z=22.6$ and $J=21.0 \mathrm{mag}$ ) focusing on a circular area of 2798.4 $\operatorname{arcmin}^{2}$ around the young $\sigma$ Orionis star cluster $(\sim 3 \mathrm{Myr}, \sim 352 \mathrm{pc}$, and solar metallicity). The combination of the VISTA photometry with optical, WISE and Spitzer data allows us to identify a total of $210 \sigma$ Orionis member candidates with masses in the interval $0.25-0.004 M_{\odot}, 23$ of which are new planetary-mass object findings. These
\end{abstract}

\footnotetext{
${ }^{1}$ Departamento de Astrofísica, Universidad de La Laguna, E-38205 La Laguna, Tenerife, Spain.
} 
discoveries double the number of cluster planetary-mass candidates known so far. One object has colors compatible with a $\mathrm{T}$ spectral type. The $\sigma$ Orionis cluster harbors about as many brown dwarfs $\left(69,0.072-0.012 M_{\odot}\right)$ and planetary-mass objects $(37$, 0.012-0.004 $\left.M_{\odot}\right)$ as very low-mass stars $\left(104,0.25-0.072 M_{\odot}\right)$. Based on Spitzer data, we derive a disk frequency of $\sim 40 \%$ for very low-mass stars, brown dwarfs, and planetary mass objects in $\sigma$ Orionis. The radial density distributions of these three mass intervals are alike: all are spatially concentrated within an effective radius of $12^{\prime}(1.2 \mathrm{pc})$ around the multiple star $\sigma$ Ori, and no obvious segregation between disk-bearing and diskless objects is observed. Using the VISTA data and the Mayrit catalog, we derive the cluster mass spectrum $\left(\Delta N / \Delta M \sim M^{-\alpha}\right)$ from $\sim 19$ to $0.006 M_{\odot}$ (VISTA $Z J$ completeness), which is reasonably described by two power-law expressions with indices of $\alpha=1.7 \pm 0.2$ for $M>0.35 M_{\odot}$, and $\alpha=0.6 \pm 0.2$ for $M<0.35 M_{\odot}$. The $\sigma$ Orionis mass spectrum smoothly extends into the planetary-mass regime down to $0.004 M_{\odot}$. Our findings of T-type sources $\left(<0.004 M_{\odot}\right)$ in the VISTA $\sigma$ Orionis exploration appear to be smaller than what is predicted by the extrapolation of the cluster mass spectrum down to the survey $J$-band completeness.

Subject headings: stars: brown dwarfs, circumstellar matter, low mass, luminosity function, mass function - Galaxy: open clusters and associations: individual: ( $\sigma$ Orionis) — infrared: stars

\section{Introduction}

The stellar and substellar mass function (Salpeter 1955; see Bastian et al. 2010 for a recent review) is the most general outcome of the process of star formation in a given region. Young stellar clusters (ages less than about $10 \mathrm{Myr}$ ) offer the opportunity to study the mass function soon after the main burst of star formation activity has ceased. Clusters provide a snapshot of a roughly coeval population that has emerged from the same molecular cloud. Several of these regions stand out for their properties of proximity, age, low internal extinction, and rich number of members.

The $\sigma$ Orionis cluster ( $\sim 3 \mathrm{Myr}$, Zapatero Osorio et al. 2002b; Sherry et al. 2008; $300-450 \mathrm{pc}^{1}$, Brown et al. 1994; Perryman et al. 1997; Mayne \& Naylor 2008; Sherry et al. 2008; Caballero 2008a; $E(B-V)=0.05 \mathrm{mag}$, Lee 1968; and solar metallicity, González Hernández et al. 2008) is known since the early studies of Garrison (1967) and Lyngå (1981, 1983). X-ray observations of the region around the $\sigma$ Ori multiple star revealed a very young stellar population (Wolk 1996; Walter et al. 1997). The $\sigma$ Orionis cluster is acknowledged as one suitable ground where observational efforts seek to establish to what extent there is a continuity of the star formation process from the OB-type stars (the star $\sigma$ Ori itself is a multiple, massive system in the main sequence) down to free-floating

\footnotetext{
${ }^{1}$ Here, we will adopt the Hipparcos distance, 352 pc, measured by Perryman et al. (1997).
} 
planetary mass objects of a few times the mass of Jupiter ${ }^{2}$ (e.g., see the Mayrit catalog by Caballero $2008 \mathrm{c}$ and references therein).

The stellar mass function of the $\sigma$ Orionis cluster in the mass range 1-24 $M_{\odot}$ was discussed in Caballero (2007b). This author found it consistent with the Salpeter mass function (Salpeter 1955). Regarding the substellar domain, the first $\sigma$ Orionis brown dwarf discoveries were reported about a decade ago (Béjar et al. 1999; Zapatero Osorio et al. 2000). Since then, combined deep optical and near-infrared photometric surveys have led to the detection of hundreds of cluster low-mass stars, brown dwarfs, and planetary-mass candidates (with a mass below the deuterium burning-mass limit at $\left.13 M_{\text {Jup }}\right)$ down to about $5 M_{\text {Jup }}$; a considerable fraction of them $(\sim 70 \%)$ have follow-up spectroscopy and mid-infrared flux excesses confirming their likely membership in the cluster; (e.g., Martín et al. 2001; Barrado y Navascués et al. 2001, 2002b; Zapatero Osorio et al. 2002a,b; Jeffries et al. 2006; Hernández et al. 2007; Zapatero Osorio et al. 2007; Caballero et al. 2007, 2008b; Sacco et al. 2007, 2008; Scholz \& Jayawardhana 2008; Luhman et al. 2008; Rigliaco et al. 2009). The first derivation of the $\sigma$ Orionis low-mass function $\left(0.2-0.013 M_{\odot}\right)$ was presented in Béjar et al. (2001) covering an area of $0.24 \mathrm{deg}^{2}$.

Only two surveys (Lodieu et al. 2009; Béjar et al. 2011) have homogeneously explored a large area in the $\sigma$ Orionis cluster $\left(0.8-1 \mathrm{deg}^{2}\right)$ down to the brown dwarf-planet borderline. These authors found that the cluster mass spectrum in the mass interval from low-mass stars to about $13 M_{\text {Jup }}$ can be fit by a power-law expression $\Delta N / \Delta M \sim M^{-\alpha}$, where $\alpha=0.5 \pm 0.2$ (Lodieu et al. 2009) and $0.7 \pm 0.3$ (Béjar et al. 2011) [Salpeter's $\alpha=2.35$ ].

Power law functions have been found appropriate to describe the mass function of several open clusters over the mass range 0.6-0.04 $M_{\odot}$, like Upper Scorpius ( 5 Myr, Lodieu et al. 2007b), IC 4665 ( $\sim 25 \mathrm{Myr}$, de Wit et al. 2006; Lodieu et al. 2011), the Pleiades ( 120 Myr, e.g., Zapatero Osorio et al. 1997; Bouvier et al. 1998; Martín et al. 2000; Bihain et al. 2006; Lodieu et al. 2007a), Blanco I ( 130 Myr, Moraux et al. 2007), the Orion Nebula Cluster ( 1 Myr, Muench et al. 2002 and references therein) and, the field (Bastian et al. 2010 and references therein). The extension of the $\sigma$ Orionis mass function toward a smaller mass ( $\left.\sim 6 M_{\mathrm{Jup}}\right)$ in tiny regions of the cluster yielded $\alpha$ values in the range 0.4-0.6 (González-García et al. 2006; Caballero et al. 2007). Bihain et al. (2009) suggested that there may exist a turnover in the substellar mass spectrum of $\sigma$ Orionis below $6 M_{\mathrm{Jup}}$.

Here, we present VISTA (Visible and Infrared Survey Telescope for Astronomy) observations of $\sigma$ Orionis using broadband filters $Z Y J H K_{s}$ aiming at defining the substellar cluster sequence and its global properties in a consistent manner. One noteworthy characteristic of this survey is its homogeneity in mass coverage from 0.25 to $0.006 M_{\odot}\left(\sim 262-6 M_{\mathrm{Jup}}\right.$, completeness $)$ all across an important fraction of the cluster area and uncovering $\geq 75 \%$ of cluster members. VISTA and complementary photometry, and the identification of cluster member candidates are shown in Sections

\footnotetext{
${ }^{2} 1 M_{\odot}=1047 M_{\text {Jup }}$
} 
2 and 3. Mass estimates based on theoretical isochrones are presented in Section 4. In Section 5 we discuss the object contamination in the VISTA survey. By combining VISTA data with photometry from the literature, we study the presence of mid-infrared flux excesses in Section 6. Section 7 deals with the spatial distribution of very low-mass stars, brown dwarfs and free-floating planets in $\sigma$ Orionis. Finally, in Section 8 we discuss the cluster mass function from the O-type stars through the free-floating planets. Conclusions are presented in Section 9.

\section{Observations}

\subsection{Near-infrared photometry: VISTA}

Our survey of the $\sigma$ Orionis cluster is based on the VISTA Orion survey data (Petr-Gotzens et al. 2011). This survey was a dedicated imaging exploration of $30 \mathrm{deg}^{2}$ in the Orion Belt region, including $\sigma$ Orionis, carried out as part of the VISTA science verification program. VISTA (Dalton et al. 2006; Emerson et al. 2006) is a near-infrared survey facility equipped with a wide-field, nearinfrared camera (VIRCAM) and mounted on a 4.2-m telescope at ESO's Paranal Observatory. VIRCAM comprises sixteen $2048 \times 2048$ pixel infrared detectors (Raytheon VIRGO HgCdTe) with a mean pixel size of $0^{\prime \prime} 339$ and with signicant interchip gaps. Therefore, the area covered by one VIRCAM exposure (or "pawprint") is $0.6 \mathrm{deg}^{2}$ in a field of view of $1.3 \times 1.0 \mathrm{deg}^{2}$. For a uniform sky coverage, six pawprints offset by a convenient amount are required to fill an area of approximately $1.5 \times 1.1 \mathrm{deg}^{2}$, which is called a "tile". For details on the VISTA Orion survey strategy, number of observed tiles, and a description of the data sets we refer to Arnaboldi et al. (2010) and Petr-Gotzens et al. (2011).

Observations of the $\sigma$ Orionis region (tile number 16 according to the nomenclature of the VISTA Orion survey) were performed between 2009 October 20 and October 28 employing the VISTA broadband filters $Z Y J H K_{s}$. The integration times at $Z$ - and $J$-band were roughly twice and 5 times, respectively, longer than what was used for the overall VISTA Orion survey, allowing us to obtain deeper images. This was crucial in order to identify the lowest mass cluster member candidates in $\sigma$ Orionis. In Table 1 we provide the specific observing dates, total exposure times per filter, and seeing conditions as measured from the full width at half maximum (FWHM) of point-like sources in the stacked mosaics.

Given the large amount of the VISTA Orion data ( 559 Gb), they were processed by a dedicated pipeline run by the Cambridge Astronomical Survey Unit (CASU) ${ }^{3}$. We used the reduced products obtained with the VIRCAM pipeline version 1.0, which delivered science-ready stacked images and mosaics, as well as photometrically and astrometrically calibrated source catalogs. Standard reduction steps included dark and flat-field corrections, and sky background subtraction.

\footnotetext{
${ }^{3}$ http://casu.ast.cam.ac.uk
} 
All individual images (pawprints) were aligned and stacked together, and then conveniently montaged to produce deep tiles. Automatic identification of sources and photometry were performed by the instrument pipeline in CASU. Tile images of different filters were treated individually. Aperture photometry with an aperture radius of 2 .'0 was obtained to avoid contamination from nearby sources. Aperture corrections were applied. Instrumental $J H K_{s}$ magnitudes were converted into apparent calibrated magnitudes using the 2MASS photometry (Skrutskie et al. 2006), and the $Z Y$ data were calibrated using routine observations of UKIRT photometric standard star fields (Casali et al. 2007) carried out during the Orion survey campaign. The photometric calibration precision is typically better than $5 \%$ for magnitudes brighter than the completeness magnitude. The 2MASS catalog was also used to calibrate astrometrically the VISTA Orion data to an absolute precision of $0 \prime \prime 3$ (relative astrometric accuracy of $0 ! 1$ ). Data of different filters were paired within a 2 '.0 radius to produce a merged catalog (both non- and cross-correlated objects are included).

We adopted the completeness magnitude of the VISTA $\sigma$ Orionis survey as the faintest magnitude at which the number of sources per interval of magnitude does not deviate from an increasing distribution. This function is obtained from the fit of an exponential distribution from bright to intermediate magnitudes in each passband. We adopted the limiting magnitude as the magnitude bin at which the total number of sources deviates by $\geq 50 \%$ from the prediction of the exponential law. Our determined completeness and limiting magnitudes (see Table 1) roughly correspond to source detections around the 10- $\sigma$ and 4- $\sigma$ level, respectively ( $\sigma$ is the sky-subtracted background noise). At the bright end of the survey, we considered sources with $J \geq 13$ mag to ensure that the registered photon counts laid within the linearity regime of the VIRCAM detectors in all filters.

In next sections we present the cross-correlation of the VISTA $\sigma$ Orionis catalog with various other catalogs compiled by us or taken from the literature. We used a cross-correlation radius of 2 .'0, which takes into account the astrometric uncertainties of the catalogs and possible systematic deviations.

\subsection{Mid-infrared photometry}

\subsubsection{Spitzer}

To extend the VISTA wavelength coverage toward the mid-infrared wavelengths, and aimed at studying the presence of mid-infrared flux excesses in $\sigma$ Orionis objects, we complemented the VISTA data set with [3.6], [4.5], [5.8], and [8.0]-band photometry acquired with the Spitzer Space Telescope Infrared Array Camera (IRAC, Fazio et al. 2004). The four-channel Spitzer data were collected by Hernández et al. (2007) on 2004 October 9 (Guaranteed Time Observation program \#37, PI: G. Fazio), and covered an area of $\sim 1739 \operatorname{arcmin}^{2}$ in the $\sigma$ Orionis cluster. These data have been widely used by different groups (Caballero et al. 2007; Zapatero Osorio et al. 2007; Scholz \& Jayawardhana 2008; Luhman et al. 2008). The completeness limits are 17.25, 17.0, 14.75, and $14.0 \mathrm{mag}$ at $3.6,4.5,5.8$, and $8.0 \mu \mathrm{m}$, respectively (Luhman et al. 2008). VISTA data (particularly 
the $J$-band) are sufficiently sensitive to ensure the detection of the cluster sources to the same depth (i.e., low mass objects), or even beyond, as the Spitzer images. Here, we employed the Spitzer photometry obtained by Zapatero Osorio et al. (2007). Figure 1 shows the location of the Spitzer/IRAC fields, which are contained in the VISTA area but cover only $\sim 62 \%$ of the explored circular region in $\sigma$ Orionis.

\subsubsection{WISE}

Additional mid-infrared photometry at 3.4, 4.6, 12, and $22 \mu \mathrm{m}(W 1, W 2, W 3, W 4)$ was provided by the Wide-field Infrared Survey Explorer (WISE) space mission (Wright et al. 2010). These data were extracted from the WISE preliminary data release ${ }^{4}$, which includes the first 105 days of WISE survey observations between 2010 January 14 and 2010 April 29. WISE photometry is processed with initial calibrations and reduction algorithms. Astrometric accuracy of sources brighter than $W 1 \sim 13.0 \mathrm{mag}$ is $0^{\prime \prime} \cdot 2$. From the on-line WISE catalog, we retrieved magnitudes of cross-correlated VISTA sources with quoted mid-infrared photometric signal-to-noise $(\mathrm{S} / \mathrm{N})$ ratio above 4. The quoted 5- $\sigma$ WISE detection limits are 15.3, 14.4, 10.1 and $6.7 \mathrm{mag}$ at $W 1, W 2$, $W 3$ and $W 4$, respectively. In addition, we inspected WISE images visually for all cross-correlated sources. The VISTA survey and the Spitzer images are significantly deeper than the WISE data. In contrast to Spitzer, WISE covered the full extension of the $\sigma$ Orionis cluster. The combination of VISTA and WISE photometry (Section 6) will allow us to study the presence of mid-infrared flux excesses of very low-mass stars and brown dwarfs all across the cluster area.

\subsection{Optical photometry}

The combination of optical and near-infrared data is notably useful to distinguish true ultracool late-L and T-type dwarfs from photometric contaminants. Ideally, the VISTA survey should have been complemented with red optical images down to $I=25 \mathrm{mag}$ or $Z=24 \mathrm{mag}$, which would have enabled us to detect the latest L- and early- to mid-T members of $\sigma$ Orionis at visible wavelengths. Unfortunately, we do not have these very deep optical images. We used all optical data available to us, including published and new photometry.

The images taken with the Wide Field Camera (WFC) of the 2.5-m Isaac Newton Telescope on La Palma Island cover a total area of $970 \operatorname{arcmin}^{2}$ around the cluster center (Caballero et al. 2007). We refer to this reference for further details on the WFC photometry. The authors reported a limiting magnitude of $I=24.1 \mathrm{mag}$.

We also employed unpublished data taken by us with the Suprime-Cam mounted on the prime

\footnotetext{
${ }^{4}$ http://wise2.ipac.caltech.edu/docs/release/prelim/
} 
focus of the 8-m Subaru telescope on Hawaii. Observations were conducted using the Sloan $z^{\prime}$ and Cousins $I$ filters on 2000 December 28. These filters, centered at $\sim 800 \mathrm{~nm}\left(I_{c}\right)$ and $\sim 910 \mathrm{~nm}$ $\left(z^{\prime}\right)$, are appropriate to characterize very red and cool sources. Suprime-Cam had a mosaic of ten MIT/LL $2048 \times 4096$-pixel CCDs arranged in a pattern of $5 \times 2$ with interchip gaps of about 16 ". 5 in its "old" version prior to 2008. The projection of one pixel onto the sky is 0 ' 2 . At the time of the observations, the South-East CCD (number six) was dead and did not register any images. A total of five $\left(z^{\prime}\right)$ and ten $\left(I_{c}\right)$ exposures of 240 s each were acquired with dither offsets of $\sim 20^{\prime \prime}$ for a proper removal of the telluric background contribution. Therefore, total integration times were 20 $\min \left(z^{\prime}\right)$ and $40 \mathrm{~min}\left(I_{c}\right)$. Taking into account the overlapping area of the dithers and the size of the nine (useful) detectors, our Suprime-Cam observations provided a field of view of $570 \mathrm{arcmin}^{2}$ North of the cluster center. In Table 2 we list the measured central coordinates of each of the nine detectors. Observations were carried out under good conditions of sky transparency and seeing of $0 ! 9$ ( $z^{\prime}$ band).

Raw frames were bias-subtracted and flat-field corrected using packages running inside the IRAF environment. We constructed the flat-field images for each passband by combining all SuprimeCam (adequately scaled) science images taken during the night. Individual processed frames were conveniently aligned and stacked together to produce deep images.

The photometric aperture and point-spread function fitting analysis was carried out using routines within DАОРНОТ, which provides image profile information needed to discriminate between stars and resolved sources. Instrumental $I_{c}$ magnitudes were transformed into observed magnitudes using WFC observations by Béjar et al. (2011): more than 190 sources with photometric error bars $\leq 0.15 \mathrm{mag}$ were in common to both WFC and Suprime-Cam searches. We found that the uncertainty in the photometric calibration of the $I_{c}$ band is $\sim 0.03$ mag. Unfortunately, no standard stars were observed in the Sloan $z^{\prime}$ filter for a proper calibration of the data. Therefore, we have not performed an absolute calibration of this passband, but have constructed color-magnitude diagrams where calibrated $I_{c}$ is plotted against the semi-instrumental index $I_{c}-z^{\prime}$. Sources with intrinsically red colors will stand out toward high values of $I_{c}-z^{\prime}$ with respect to the great bulk of field objects. Completeness and limiting magnitudes of the Suprime-Cam survey were determined following the procedure indicated in Section 2.1. We have estimated them to be $I_{c}=22.5 \mathrm{mag}$ for the completeness $(10-\sigma)$, and $I_{c}=23.8 \mathrm{mag}$ for the limit (4- $\left.\sigma\right)$. All Suprime-Cam CCDs were astrometrically calibrated using 2 MASS data to an accuracy of $0{ }^{\prime \prime} 1-0{ }^{\prime \prime} 3$.

\section{Selection of cluster member candidates}

\subsection{Search area definition}

We searched for $\sigma$ Orionis photometric member candidates in a circular area of radius $30^{\prime}$ centered on the massive star of the same name. Béjar et al. (2004a, 2011), Caballero (2007b, 2008c) and Lodieu et al. (2009) have discussed that the cluster sequence is clearly distinguished from the 
field sources up to $30^{\prime}$. At larger radii, the contamination by other objects (e.g., young Orion sources, foreground and background sources) increases notably, blurring the $\sigma$ Orionis sequence. Caballero (2008b) describes the cluster as a dense core extending from the center to a radius of $20^{\prime}$ and a rarified halo at larger separations up to $30^{\prime}$. In addition, Béjar et al. (2011) observed that at radii larger than $30^{\prime}$ there is an overdensity of red objects to the North and West of the cluster that might be related to the young population of the Orion Belt. The extension of $\sigma$ Orionis is addressed in Section 7. As illustrated in Figure 1, the 30'-radius circular region of our search is contained within tile 16 of the VISTA Orion survey except for a small sector of $\sim 12 \operatorname{arcmin}^{2}$ to the North of the cluster. This sector is covered by other tiles of the VISTA survey, but they are not as deep as tile 16 and are therefore not included.

Within the circular area there exist massive $\mathrm{O}, \mathrm{B}$, and A-type stars strongly saturating all optical and infrared data. Their bright "halos" extend beyond a few times the point-spread function (PSF) of the images, artificially increasing the noise of the local background level and preventing us from detecting very faint (low-mass) sources in their surroundings. We estimated that the area lost to this effect is $17.0 \mathrm{arcmin}^{2}$. We determined the final deep area of our VISTA survey to be $2798.4 \operatorname{arcmin}^{2}\left(0.78 \mathrm{deg}^{2}\right)$.

\subsection{VISTA $Z J$ color-magnitude diagram}

Since we sought cool objects with red $Z-J$ colors and the VISTA $Z$ and $J$-band images are quite deep, we constructed the $J$ vs. $Z-J$ color-magnitude diagram to select photometrically $\sigma$ Orionis member candidates. Previously known cluster members trace the cluster sequence from $J=13$ down to 20 mag: there are 145 of these objects in the literature cross-correlated with VISTA and whose young age is confirmed by means of spectroscopic and mid-infrared photometric observations. As evidence for youth we considered the following signatures: the detection of the Li I doublet atomic absorption at $670.8 \mathrm{~nm}$ (Zapatero Osorio et al. 2002b; Barrado y Navascués et al. 2003; Kenyon et al. 2005; Sacco et al. 2008), broad or strong $\mathrm{H} \alpha$ emission compatible with accretion events (Haro \& Moreno 1953; Wiramihardja et al. 1989, 1991; Barrado y Navascués et al. 2001; Zapatero Osorio et al. 2002b; Barrado y Navascués et al. 2002b, 2003; Andrews et al. 2004; Weaver \& Babcock 2004; Kenyon et al. 2005; Sacco et al. 2008), weak alkali absorption lines associated to low-gravity atmospheres (Béjar et al. 1999; McGovern et al. 2004; Kenyon et al. 2005; Burningham et al. 2005; Maxted et al. 2008), radial velocity consistent with the cluster systemic velocity, $\sim 30 \mathrm{~km} \mathrm{~s}^{-1}$, (Kenyon et al. 2005; Burningham et al. 2005; Maxted et al. 2008; Sacco et al. 2008), and/or infrared flux excesses indicative of the presence of surrounding disks or envelopes (Barrado y Navascués et al. 2003; Jayawardhana et al. 2003; Oliveira et al. 2006; Hernández et al. 2007; Caballero et al. 2007; Zapatero Osorio et al. 2007; Scholz \& Jayawardhana 2008; Luhman

et al. 2008). The $Z J$ sequence delineated by these $145 \sigma$ Orionis members is shown in Figure 2 (blue filled circles). This population is clearly distinguishable from field sources (small dots) since, for a given magnitude, $\sigma$ Orionis true members have redder $Z-J$ colors. 
To select new photometric member candidates we traced a blue envelope along the known cluster sequence. This blue envelope goes from $(Z-J, J)=(0.93,13.0)$ to $(2.6,20.5) \mathrm{mag}$ and is plotted as a solid line in Figure 2. It separates field sources from eligible $\sigma$ Orionis member candidates. The so-defined blue envelope roughly corresponds to a linear fit of the cluster $Z J$ sequence shifted at $\sim 2-\sigma$ (where $\sigma$ is the color dispersion of the fit) toward blue $Z-J$ indices. Of the 145 original sources, 129 are located within the eligible zone (red side of the envelope, see Table 3 ). Most of the cluster members that lie outside of the eligible zone have $J \leq 16$ mag, they represent about $10 \%$ of the confirmed young cluster members, i.e., by using the defined blue envelope we might be including the $90 \%$ of potential cluster members in the interval $J=13-16$ mag.

For the magnitude range $J=13-20.5$ mag, where $J \sim 20.5$ mag matches the VISTA survey limiting $Z$-band magnitude of cluster members, 219 VISTA sources comply with our $Z J$ photometric criterion for $\sigma$ Orionis membership. Of them, 129 are cluster members with confirmed features of youth (see above), 58 are reported in the literature as photometric candidates yet pending spectroscopic confirmation of their membership, and 32 are new detections. After visual inspection of the VISTA images, we rejected 12 candidates out of the 32 new sources: nine are merged bright sources, two are spurious detections, and one object is spatially resolved (its FWHM is larger than the average). The VISTA photometry of the resolved object is provided in Table 4. Here we report the finding of $207 \sigma$ Orionis members and member candidates, 20 of which are new (red symbols in Figure 2). All new detections have $J$-band brightness in the interval 18-20.5 mag, which according to evolutionary models (Chabrier et al. 2000; Baraffe et al. 2003) corresponds to a mass range of $\sim 0.011-0.004 M_{\odot}$ at the adopted age and distance of the cluster, i.e., at the planet-brown dwarf boundary and below. There are no new more massive $\sigma$ Orionis candidates in the VISTA survey, indicating that previous works (Béjar et al. 1999, 2001, 2011; Zapatero Osorio et al. 2000; González-García et al. 2006; Caballero et al. 2007; Lodieu et al. 2009; Bihain et al. 2009) carried out a successful census of the cluster very low-mass star and brown dwarf populations.

One new cluster member candidate, S Ori J053804.65-021352.5, lies at about 1".0 north of a field source. To secure the VISTA photometry, we performed PSF photometric analysis (using the DAOPHOT package) particularly for the $Z$ band, where the candidate is significantly fainter than the field object. The $Z-J$ color of S Ori J053804.65-021352.5 suggests a T spectral type; this source is the only T-type candidate detected at both $Z$ and $J$ bands in the VISTA $\sigma$ Orionis survey.

In Table 5 we provide the VISTA, Spitzer, and WISE photometry of the $145 \sigma$ Orionis candidates members with youth features (129 of them within our $Z J$ eligible zone). The observational evidence for their cluster membership along with further information, including bibliography, and an indication of whether they lie to the red side of the blue envelope in the $J$ vs. $Z-J$ diagram are given in Table 3. The VISTA, Spitzer, and WISE photometry of the $58 \sigma$ Orionis member candidates known in the literature that lie in the eligible zone of the $Z J$ diagram is listed in Table 6 , and Table 7 provides additional information for them. The data of the 20 new planetary-mass candidates reported here are given in Table 8, 90\% of them remain undetected in the WISE dataset. 


\subsection{Additional color-magnitude diagrams}

To test the quality of the $Z J$ candidates as well as to delineate the $\sigma$ Orionis sequence at other wavelengths, we built various color-magnitude diagrams using the VISTA data. Figure 3 depicts the $J$ magnitudes as a function of $J-H$, and $J-K_{s}$. We also included the combined VISTA and Spitzer $J$ vs. $J-[3.6]$ and $J-[4.5]$ diagrams in Figure 3. For comparison purposes, we overlaid the average field sequence of mid-M to T-type objects (typical ages 1-5 Gyr) after normalization to the late-M population of the $\sigma$ Orionis cluster. We constructed the average field sequence by calculating the mean magnitudes and colors for each spectral type and using the data published by Hewett et al. (2006), Patten et al. (2006) and Leggett et al. (2007).

All $Z J$ objects nicely describe a continuous photometric sequence in the four color-magnitude diagrams of Figure 3. With the only exception of S Ori J053804.65-021352.5, none appears to deviate toward blue colors, which would have made us reject it as a cluster member candidate. According to Figure 3, most of the new candidates show colors typical of early-L through late-L types. S Ori J053804.65-021352.5 has colors (from $Z$ through [3.6]) fully compatible with T0-T4 types. Unfortunately, there is no [4.5] photometry for this source since its location was not covered by the corresponding Spitzer image. In short, all possible color-magnitude diagrams covering the wavelength range 0.9-4.5 $\mu \mathrm{m}$ confirm the photometric cluster member candidacy of the 207 sources originally selected from the VISTA $Z J$ data in the interval $J=13-20.5$ mag.

\subsection{Extended search down to $J$ completeness}

The $Z J$ search is limited by the $Z$ band. To fully exploit the deep VISTA $J$-band data, we extended the search for $\sigma$ Orionis member candidates down to $J=21 \mathrm{mag}$, which is the estimated completeness magnitude of the survey in this near-infrared band. According to Figure 3, at these faint magnitudes we would expect cluster members with colors typical of late-L and $\mathrm{T}$ types. The following photometric criteria, optimized for very cool objects, were applied:

- We identified all sources with no $Z$ detections and $J$ magnitudes in the interval 19-21 mag present in the VISTA $\sigma$ Orionis catalog. This criterion would also allow us to "recover" those objects with $Z$ magnitudes between completeness and detection limit that might have been lost by the automatic source identification of the instrument pipeline. This resulted in over a thousand potential candidates.

- After visual inspection of the $J$-band images, we were able to reject most of them since they turned out to be spurious detections or detector defects. The $Z$-band images were also inspected and we discarded those objects with $Z$-band fluxes above the 10- $\sigma$ detection (the $Z$-band automatic identification failed particularly on those objects close to bright stars). Basically, we were interested in sources with $Z-J \geq 2.1 \mathrm{mag}$ (as illustrated in Figure 2) since it is expected that true cluster members increase their $Z-J$ color at fainter magnitudes 
(or lower masses).

- Other filters were used to impose additional constraints: the VISTA $Y$-band and Spitzer (VISTA $H K_{s}$ images are not deep enough for our purposes). For those objects with positive detections in any of the filters we considered the following color cuts: $Y-J \geq 0.6, J-[3.6] \leq$ 3.5 , and $J-[4.5] \leq 4.5 \mathrm{mag}$. All color criteria have to be satisfied simultaneously. They allowed us to reject highly reddened extragalactic sources and Galactic objects earlier than M types. Figure 1 of Burningham et al. (2010) showed that L and T type sources have $Y-J$ colors in the range $0.5-1.7 \mathrm{mag}$; these authors also discussed that the $Y-J \geq 0.5 \mathrm{mag}$ criterion should not exclude any T type objects. Since the Spitzer color cuts are based on the typical colors of $\mathrm{L}$ and $\mathrm{T}$ type sources in the field (Patten et al. 2006), we increased them generously to account for any possible mid-infrared flux excess present in cluster members. Those candidates from previous steps with non detections in $Y$, [3.6], and [4.5] were kept in the pool. At this stage, the number of potential candidates is reduced to 82 .

- Finally, we used the optical data set and imposed $I-J \geq 3.0$ mag. This step left us 65 new potential candidates. Of the 17 rejected objects, 12 have WFC photometry, and five have Suprime-Cam data.

After applying these criteria, we successfully recovered the known T dwarfs S Ori 70 (T5.5, Zapatero Osorio et al. 2002a) and S Ori 73 (T4, Bihain et al. 2009; Peña Ramírez et al. 2011) in the direction toward the $\sigma$ Orionis cluster. SOri 70 is detected at both $Y$ and $J$, while SOri 73 is detected only in the $J$-band (Peña Ramírez et al. 2011). We are confident that our extended search should have identified all possible T-type sources in the surveyed area down to $J=21$ mag. However, we imposed color cuts based on those of field L and T dwarfs, which have high gravity atmospheres. If the colors of T-type sources were proven to dramatically depend on surface gravity, the selection photometric criteria should have to be revised.

Of the 65 potential candidates, 35 have visual detections in the $Z$ band. We obtained their PSF photometry and/or photometry based on the peak count ratios. All have $Z$ magnitudes between the completeness and the limiting magnitudes of the survey. Only six sources fulfilled our $Z-J$ color cut, three of which were resolved (see Table 4) and the other three remain unresolved (star-like FWHM). The unresolved candidates are S Ori J054017.3-023623, S Ori J053923.3-021235 and S Ori J053716.8-024308, and appear as red symbols in Figures 2 and 3. We provide their photometry in Table 8. All their colors (two of them have Spitzer photometry) are compatible with the L types.

These three sources add to the 20 new candidates found in Section 3.2. Therefore, in the VISTA survey we found a total of $210(=207+3) \sigma$ Orionis member candidates with $Z J$ detections $(J=13-20.5 \mathrm{mag})$, and 30 with $J$-band detection $(J=20.5-21 \mathrm{mag})$ and $Z-J \geq 2.1 \mathrm{mag}$ (Table 9). As we will discuss in Sections 5 and 7, the group of 30 suffers from high object contamination, and it will not be included in the study of the cluster mass function. 
Of the 65 potential candidates, 30 have $Z$-band magnitudes beyond the limit of the survey $(Z \geq 23.1 \mathrm{mag})$ and are not previously reported in the literature. They are listed in Table 9 and plotted as red arrows in Figure 2. Of the 30, 29 have a positive detection in another filter, and their colors are consistent with the L-types. One source is detected only in the $J$ band.

Finally, in the surveyed area of $2798.4 \operatorname{arcmin}^{2}$ there are only three sources with colors compatible with $\mathrm{T}$ spectral types (i.e., significant methane absorption at near-infrared wavelengths), two of which are known (S Ori70 and 73), and the third one is a new candidate reported here (S Ori J053804.6-021352). In addition, there is a source detected only in the $J$-band; we cannot conclude on its likely nature.

\subsection{Known objects out of the $Z J$ selection}

With some exceptions, our search recovered all previously known $\sigma$ Orionis confirmed members and member candidates with magnitudes in the interval $J=16-21$ mag (well within the substellar domain and redder than our $Z J$ blue envelope). Below we briefly discuss those exceptions; we also include S Ori70, 72, and 73, which, although found in the extended survey as $Z$-band nondetections, are not considered in the cluster mass function analysis. The photometry of the objects indicated next is given in Table 5 (those with features of youth) and Table 10 (remaining sources).

- S Ori 55 (Zapatero Osorio et al. 2000) is an M9-type substellar member of the cluster with very intense and variable $\mathrm{H} \alpha$ emission (Zapatero Osorio et al. 2002b). It has a VISTA $Z-J$ color slightly bluer than our photometric color cut, and it lies very close to the blue envelope depicted in Figure 2. We remark that S Ori 55 follows the $\sigma$ Orionis photometric sequence in all other color-magnitude diagrams shown in Figure 3.

- S Ori57 (Zapatero Osorio et al. 2000) was rejected as a cluster photometric candidate by Martín et al. (2001) based on near-infrared photometry. Our VISTA data confirm that this object deviates from the cluster photometric sequence in all of the color-magnitude diagrams of Figures 2 and 3 with the only exception of the $J$ vs. $J-H$ diagram.

- S Ori 61 (Zapatero Osorio et al. 2000) was found to have an optical spectral type inconsistent with cluster membership by Barrado y Navascués et al. (2001), and it was rejected as a cluster member candidate. With VISTA this object was only detected in the $Z$ band with a large error bar.

- S Ori 64 (Zapatero Osorio et al. 2000) is one of a few low-mass candidates lacking follow-up spectroscopy to assess cluster membership. It was detected in all five VISTA filters; its $Z-J$ color is slightly bluer than our photometric color cut.

- S Ori 65 (Zapatero Osorio et al. 2000) was assigned a spectral type of L3.5 by Barrado y Navascués et al. (2001). It follows the $\sigma$ Orionis spectroscopic sequence. More recently, 
Scholz \& Jayawardhana (2008) suggested that S Ori 65 has a flux excess at $8 \mu$ m indicative of a surrounding disk. Luhman et al. (2008) did not reach the same conclusion arguing that the object photometric precision was insufficient for reliably detecting excess emission. S Ori 65 has a slightly blue VISTA $Z-J$ color, but it nicely sits on top of the cluster photometric sequence in the color-magnitude diagrams of Figure 3. VISTA $J$ magnitude and the data from the literature differ by $0.4 \mathrm{mag}$. Significant veiling at optical wavelengths, strong variability and/or underestimated photometric errors of magnitudes provided in the literature might provide an explanation for its blue VISTA $Z-J$ index.

- S Ori 67 (Zapatero Osorio et al. 2000) was typed as an L5 $\sigma$ Orionis source by Barrado y Navascués et al. (2001). It follows the $\sigma$ Orionis spectroscopic sequence. All VISTA colors locate S Ori 67 very close to the photometric sequence of the cluster, but always slightly to the blue side. For this particular source, the VISTA error bars are relatively large.

- S Ori69 was the faintest candidate in Zapatero Osorio et al. (2000) survey. Martín et al. (2001) tentatively classified it as a T0 source based on a rather noisy near-infrared spectrum. Caballero et al. (2007) did not detect S Ori 69 in their deep survey, suggesting that this object was fainter than indicated by the discovery paper. S Ori 69 remains undetected in all VISTA images in agreement with the findings of these authors.

- S Ori 70 (Zapatero Osorio et al. 2002a) is a $\sigma$ Orionis T5.5 isolated planetary-mass candidate (the spectral type was measured from low resolution spectroscopy covering the $H$ and $K$ bands). Burgasser et al. (2004) argued that this object is probably a foreground field T67 dwarf based on $J$-band near-infrared spectroscopy. The VISTA $J$-band photometry of S Ori 70 is in agreement with the values reported in the literature (Zapatero Osorio et al. 2008). Its astrometric and photometric properties have been recently discussed in Scholz \& Jayawardhana (2008), Zapatero Osorio et al. (2008) and Peña Ramírez et al. (2011).

- S Ori 72 (Bihain et al. 2009) is detected only at VISTA $J H K_{s}$-bands. It was recovered in the extended survey. The VISTA $J$ magnitude differs by $0.56 \mathrm{mag}$ with respect to that of the discovery paper.

- S Ori 73 (Bihain et al. 2009) was confirmed to be a T4 source through the $H$-band methane imaging study by Peña Ramírez et al. (2011). Its high proper motion suggests it is a likely field interloper. Its VISTA $J$ magnitude is 0.33 mag brighter than the value given by Bihain et al. (2009) (see Peña Ramírez et al. 2011).

- S Ori 74 (Bihain et al. 2009) has VISTA colors significantly deviating from the cluster sequence in all diagrams of Figures 2 and 3. VISTA data do not support the photometric candidacy of this object.

From the discussion above, we concluded that our selection criteria are efficient in identifying $\sigma$ Orionis cluster member candidates, and that only $\leq 10 \%$ of true members might have been lost due to variability and/or photometric uncertainties. 


\section{Mass estimates for $\sigma$ Orionis member candidates}

To estimate the masses of our selected photometric cluster member candidates we compared their observed $J$-band magnitudes to various evolutionary models from the Lyon group: NextGen98 (Baraffe et al. 1998), AMES-Dusty (Chabrier et al. 2000), and AMES-Cond (Baraffe et al. 2003). We did not employ the absolute magnitudes provided by the models since it is known that they do not reproduce the observed spectra of cool sources convincingly. We converted theoretical luminosities and effective temperatures into observables by using the bolometric correction-temperature and color-temperature relations given in Golimowski et al. (2004) and Hewett et al. (2006). The models

in combination with the transformation equations cover the mass range $\sim 0.25-0.001 M_{\odot}$. The results are displayed in the various panels of Figure 3 for the cluster age of $3 \mathrm{Myr}$ and distance of 352 pc. Estimated masses for this canonical cluster age and distance are labeled in all colormagnitude diagrams of Figures 2 and 3 and will be used throughout this paper.

According to the Lyon models, our VISTA survey is complete in the mass interval 0.25$0.006 M_{\odot}$, which corresponds to $J=13-20 \mathrm{mag}$ (or completeness given by the $Z$ band). The extended survey $(J=21 \mathrm{mag})$ goes down to $0.003 M_{\odot}$. The substellar limit is located at $J=14.5 \mathrm{mag}$ $\left(\sim 0.072 M_{\odot}\right)$ and the planet-brown dwarf borderline lies at $J=18.1 \mathrm{mag}\left(13 M_{\mathrm{Jup}}\right)$. Within these magnitude intervals there are 104 very low mass stars, 69 brown dwarfs, and 37 planetary-mass objects in our VISTA $\sigma$ Orionis survey. Of the 37 planetary-mass candidates, 28 have magnitudes within the completeness limit of the exploration. We remark that the VISTA survey has doubled the number of $\sigma$ Orionis planetary-mass candidates known to date. For a cluster distance of $300 \mathrm{pc}$ and age of $3 \mathrm{Myr}$, our survey would be complete in the mass range $0.20-0.005 M_{\odot}$ (91 very low mass stars, 80 brown dwarfs, and 39 planetary mass objects). For the largest cluster distance of $450 \mathrm{pc}$, the mass coverage of the VISTA exploration would shift toward higher masses particulary in the stellar regime; the survey would be complete for the interval $0.38-0.007 M_{\odot}$ (128 very low mass stars, 53 brown dwarfs, and 29 objects with planetary masses).

\section{Contaminants}

In a pure photometric search without spectroscopic measurements to confirm the young nature of the candidates, in addition to possible errors in the source catalog magnitudes, the sample of cluster member candidates may be contaminated by objects displaying colors similar to those expected for members. Nevertheless, 129 out of the total of 210 candidates, i.e., $61 \%$, have confirmed membership in $\sigma$ Orionis (see Section 2). This indeed provides secure statistics for the study of the cluster mass function. We will discuss briefly what kind of objects are expected to be contaminating a survey like ours and will try to quantify each of them as follows.

The main sources of contaminants are Galactic M, L, and T type field objects (at all magnitudes) and reddened, unresolved galaxies (particularly dominating at faint magnitudes). As shown in Bihain et al. (2009), L dwarfs and red galaxies share similar near- and mid-infrared colors. 
However, because of their relatively blue near-infrared colors, T-type sources can be distinguished from reddened galaxies. We derived the Galactic contribution of contaminants following the study by Caballero et al. (2008a, and references therein). In the magnitude range $J=13-20.5 \mathrm{mag}$ (the $Z J$ search), we estimated the contamination due to $\mathrm{M}, \mathrm{L}$, and $\mathrm{T}$ sources to be 31 objects. These are divided as follows: 12 M4-M6 stars in the stellar domain $(J=13-14.5 \mathrm{mag}, 11.5 \%), 11 \mathrm{M} 6-\mathrm{L} 0$ sources in the brown dwarf regime $(J=14.5-18.1 \mathrm{mag}, 15.9 \%)$, and $7 \mathrm{~L} 0-\mathrm{L} 7$ plus $1 \mathrm{~T}$ dwarfs in the planetary-mass interval $(J=18.1-20.5 \mathrm{mag}, 21.6 \%)$. Regarding the extended survey, for the magnitude range $J=20.5-21 \mathrm{mag}$, the number of expected contaminants is an additional $1 \mathrm{~L}$ dwarf and $1 \mathrm{~T}$ dwarf.

Extragalactic contamination is expected to be negligible at magnitudes brighter than $J, H=18$ 19 mag since most galaxies might have been resolved in our survey given the pixel size of the detectors and seeing conditions. We checked the FWHM of all new candidates and confirmed that they fit the stellar PSF. Resolved sources (Table 4) were actually rejected and considered non-photometric member candidates. Also negligible is the contamination due to background cool (M-type) giant stars all across the magnitude range of the VISTA survey. Kirkpatrick et al. (1994) argued that these objects are relatively numerous in areas within $10 \mathrm{deg}$ of the Galactic plane. The $\sigma$ Orionis cluster is located well away from this region (at $b=-17.3 \mathrm{deg}$ ).

As for the extragalactic contamination at faint magnitudes, particularly reddened galaxies and galaxies at high redshifts, we used the GOODS-MUSIC multicolor V2.0 catalog of galaxies (Grazian et al. 2006; Santini et al. 2009), which has multi-wavelength coverage from 0.3 to $24 \mu \mathrm{m}$ and either spectroscopic or accurate photometric redshifts. This catalog extends beyond the limiting magnitudes of the VISTA survey, but it covers a smaller area on the sky by a factor of $1 / 19.5$. The GOODS-MUSIC magnitudes were converted into the Vega system using the relations by Hewett et al. (2006) and the IRAC Data Handbook V3.0. For the magnitude interval $J=13-20.5 \mathrm{mag}$, we retrieved all GOOD-MUSIC sources that have $Z-J$ colors to the red side of the blue envelope depicted in Figure 2. We found none. The situation changes dramatically for $J=20.5-21$ mag. With color cuts of $Z-J \geq 2.1$ and $Y-J \geq 0.6 \mathrm{mag}$, we retrieved a total of five galaxies in the GOODS-MUSIC area. These galaxies have redshifts of 1.3-1.6. By applying the additional color cuts of $J-[3.6] \leq 3.5$ and $J-[4.5] \leq 4.5$ mag that we imposed to our extended search in the $\sigma$ Orionis cluster, the number of galaxies reduces to three. All have colors "consistent" with the field L type dwarfs, and none resembles the $\mathrm{T}$ dwarfs. By scaling the area of the GOODSMUSIC and VISTA surveys, we estimated that 35-90 galaxies with redshifts $z=1.3-1.6$ may be contaminating our extended survey in the magnitude range $J=20.5-21 \mathrm{mag}$. This number is quite similar and even larger than the amount of $\sigma$ Orionis candidates with $Z$-band non-detections found in the extended survey, suggesting that most of the 30 sources of Table 9 could be extragalactic.

The extragalactic nature of two of these candidates is confirmed by inspecting the deep nearinfrared images of Peña Ramírez et al. (2011), particularly those with an excellent seeing of $0{ }^{\prime \prime} 3$. These authors covered an area of $120 \mathrm{arcmin}^{2}$ in the $\sigma$ Orionis cluster using the $H$ and methane filters. The two candidates overlapping with that area are resolved according to the photometric 
analysis made by the authors. Because of the high level of extragalactic contamination expected in the magnitude bin $J=20.5-21$ mag of our VISTA search, we shall not consider it in the mass function study.

\section{Infrared flux excesses: disks}

As largely discussed in the literature, infrared flux excesses of young objects are typically attributed to the presence of surrounding disks or envelopes. Figure 4 displays the color-color diagram [3.6]-[8.0] vs. $Z-J$, which gathers $128 \sigma$ Orionis members (of the 210 selected candidates) with positive detections in IRAC/Spitzer [3.6] and [8.0] bands. This Figure illustrates the color difference between sources with no infrared flux excesses $([3.6]-[8.0] \lesssim 0.5 \mathrm{mag})$ and objects that possibly host a circum(sub)stellar disk ([3.6]-[8.0] $\gtrsim 0.8 \mathrm{mag}$, (Luhman et al. 2006; Zapatero Osorio et al. 2007). The field dwarf sequence is also plotted in Figure 4; it nicely overlaps with the sequence of cluster objects with no Spitzer excesses. The field sequence roughly traces an horizontal line from blue $Z-J$ colors to the late-M/early-L types, i.e., the [3.6]-[8.0] color is nearly constant for the color interval $Z-J=1.0-2.5$ mag. Therefore, the distinction between objects with and without infrared excesses based on a constant color cut is valid for this color range. Two L-type sources with disks reported by Zapatero Osorio et al. (2000), S Ori 56 and S Ori60, have [3.6]-[8.0] colors redder than the field dwarf sequence, in agreement with the literature.

Of the 128 sources detected in IRAC/Spitzer [3.6] and [8.0] bands, 54 have [3.6]-[8.0] $\gtrsim 0.8 \mathrm{mag}$ (S Ori 56 and S Ori 60 included). We identified them as objects that probably harbor a disk. This yields a global fraction of objects with infrared excesses at $8.0 \mu \mathrm{m}$ of $42 \pm 6 \%$ (Poisson uncertainty). In the $\sigma$ Orionis low mass stellar regime $(J \sim 13-14.5 \mathrm{mag}), 31$ sources out of 75 present infrared excesses at $8.0 \mu \mathrm{m}$, translating into a stellar disk fraction of $41 \pm 8 \%$. Similarly, for cluster brown dwarfs $(J \sim 14.5-18.1 \mathrm{mag}$ ), the frequency of infrared flux excesses at $8.0 \mu \mathrm{m}$ turns out to be $39 \pm 9 \%$ (19 sources out of 49). Only four planetary mass objects were detected at 3.6 and $8.0 \mu \mathrm{m}$, all of them presenting flux excesses at the longest wavelength.

Only two objects with disks reported in the literature do not have a color [3.6]-[8.0] $\gtrsim 0.8$ mag: S Ori 7 and S Ori 8. The former was studied by Oliveira et al. (2006), who measured a flux excess in the $L^{\prime}$ band, and by Hernández et al. (2007), who classified this source as diskless based on its IRAC photometry. S Ori 8 was analyzed by Scholz \& Eislöffel (2004). These authors found a low amplitude photometric variability, which is better explained by the existence of photospheric spots rather than by a circumstellar disk. Later, Hernández et al. (2007) determined that this object may have an "evolved disk" according to its IRAC photometry.

Of the known photometric candidates of $\sigma$ Orionis, we report here the detection of flux excesses at $8 \mu \mathrm{m}$ for Mayrit 1082188 (Caballero 2008c), see Table 7. Furthermore, this source is detected at $22.0 \mu \mathrm{m}$ in the WISE catalog, supporting the existence of significant flux excesses at long wavelengths. 
Because a significant number of $\sigma$ Orionis sources in the planetary mass regime lacks detection at $8.0 \mu \mathrm{m}$, we extended the study of infrared flux excesses to a shorter wavelength where Spitzer was more sensitive: the IRAC/Spitzer $4.5 \mu \mathrm{m}$ band. Figure 5 depicts the $J-[4.5]$ vs. $Z-J$ color-color diagram where all sources with positive detections in all three bands are plotted. Objects with clear excesses at $8.0 \mu \mathrm{m}$, as deduced from their [3.6]-[8.0] colors, typically have $J-[4.5]$ indices $0.5 \mathrm{mag}$ redder than the field dwarf sequence, which is more than twice their photometric uncertainties. Therefore, we applied the following criterion for claiming an infrared flux excess at $4.5 \mu \mathrm{m}$ : for a given $Z-J$ color (or spectral type), any source with a $J-[4.5]$ color redder than the field sequence by more than $2-\sigma$ its photometric error bars may harbor a surrounding disk. According to this criterion, we derived that the global fraction of $\sigma$ Orionis candidates with infrared excesses at $4.5 \mu \mathrm{m}$ is $\geq 38 \pm 5 \%$. As for the different mass intervals, disk frequency at $4.5 \mu \mathrm{m}$ is as follows: $42 \pm 7 \%$ (very low-mass stars), $36 \pm 8 \%$ (brown dwarfs), and $\geq 31 \pm 11 \%$ (planetary mass objects). We note that $\sim \mathrm{L} 2-\mathrm{T} 0$ objects have similar $Z-J$ color but differing $J-[4.5]$ indices (see Figure $5)$. Because of this ambiguity, without a proper spectral classification of the cluster planetarymass objects we cannot properly assess the presence of infrared flux excesses at $4.5 \mu \mathrm{m}$ for some of these objects based on our defined criterion. Therefore, the derived disk fraction for $\sigma$ Orionis planetary-mass objects is a lower limit.

We also investigated the presence of infrared flux excesses in the $\sigma$ Orionis candidates using WISE data. Figure 6 shows the $W 1-W 3$ vs. $Z-J$ color-color diagram, including a total of 65 objects with positive detections in all four bands. Sources with confirmed Spitzer flux excesses are characterized by having colors $W 1-W 3>1.5 \mathrm{mag}$, while objects that presumably do not have surrounding disks show $W 1-W 3<1$ mag. Of the 65 WISE detections, 51 have a large $W 1-W 3$ color $(W 1-W 3>1.5 \mathrm{mag}$, i.e., infrared flux excess at $12 \mu \mathrm{m})$. WISE data are biased to the detection of $\sigma$ Orionis low-mass objects with infrared flux excesses. WISE performed a shallow exploration of the sky in the $W 3$ band; therefore, we expect a great majority of the $\sigma$ Orionis lowmass sources without flux excesses to remain undetected at $W 3$ because their purely photospheric emission lies well beyond the completeness limit of the WISE all-sky survey. In the high mass part of the stellar regime of our VISTA survey, all cluster stars with Spitzer flux excesses at $8.0 \mu \mathrm{m}$ also have excesses at $12 \mu \mathrm{m}$ from the WISE data (see Figure 6). Additionally, we found 20 cluster member candidates with apparent flux excesses at $12 \mu \mathrm{m}$ reported here for the first time (see Tables 3 and 7): 11 lie out of the area covered by Spitzer, 5 do not have detections in the Spitzer [3.6] and $[8.0]$ bands (and were not included in our analysis above), and another 4 do not show any flux excesses at $8 \mu \mathrm{m}$.

We caution that the disk fractions discussed above represent a lower value of the "true" disk incidence in $\sigma$ Orionis since, as stated in Section 5, we expect some object contamination in our VISTA survey. Contaminants are likely diskless, because the majority of them are expected to be old late-type field objects. In addition, cold (evolved) disks and/or disks with significant interior gaps are not detected at short wavelengths $(\leq 12 \mu \mathrm{m})$. By using IRAC/Spitzer and WISE we are missing these cold disks, particularly at the lowest masses. It is expected that the derived 
disk frequency increases with wavelength (as an example, consider the four stars, [KJN2005]8, [SWW2004]J053926.768-024258.25, S Ori J053821.3-023336, and S Ori J053853.8-024459, with clear flux excesses at $12 \mu \mathrm{m}$ and no evidence for excesses at $8 \mu \mathrm{m}$ ). In addition, Zapatero Osorio et al. (2007) pointed out that $\sigma$ Orionis planetary-mass object inner disk excess emission becomes clearly detectable longward of $5 \mu \mathrm{m}$, e.g., S Ori 60 exhibits an excess only at $8 \mu \mathrm{m}$, while its emission at shorter wavelengths is mainly photospheric.

At 4.5 and $8.0 \mu \mathrm{m}$, the derived disk fractions (i.e., infrared flux excesses) for $\sigma$ Orionis very low-mass stars, brown dwarfs, and planetary mass objects are quite alike, about 40\%, within error bars. This relatively high disk incidence suggests that the effect of evaporating inner disks through external ionization from massive O-type stars is not playing a major role in $\sigma$ Orionis low-mass members. This fraction also agrees with the derivations available in the literature (Hernández et al. 2007; Caballero et al. 2007; Zapatero Osorio et al. 2007; Scholz \& Jayawardhana 2008; Luhman et al. 2008; Béjar et al. 2011), indicating that inner/warm disks typically last for at least a few million years (Haisch et al. 2001) for both low-mass stellar and substellar regimes.

\section{Spatial distribution}

The right ascension (RA) and declination (DEC) spatial distribution of $\sigma$ Orionis sources is illustrated in Figure 7; we considered the following magnitudes intervals: very low mass stars $(J=13-14.5 \mathrm{mag})$, brown dwarfs $(J=14.5-18.1 \mathrm{mag})$, planetary mass objects $(J>18.1 \mathrm{mag})$, and $Z$-band non-detection candidates $(J>20.5 \mathrm{mag})$. Figure 8 displays the histograms of the objects spatial distribution for the RA and DEC coordinates separately. The largest concentration of cluster member candidates lies very close to the projected location of the massive, multiple star $\sigma$ Ori AB. The DEC histogram is quite symmetric at both North and South directions with respect to the position of this star. From the RA histogram, it can be seen that the number of $\sigma$ Orionis sources decreases smoothly toward the East, while the distribution drops sharply toward the West. Caballero (2008b) found a similar result and explained it as an azimuthal asymmetry due to a filament-shape overdensity of objects connecting the cluster center with a part of the Horsehead Nebula. Nevertheless, the number of objects toward the West and East of the cluster center does not differ by more than 10\%, and the number of objects in each RA, DEC quadrant is coincident within $20 \%$, i.e., the Poisson errors.

With respect to the $Z$-band non-detected candidates (Section 3.4), $~ 50 \%$ of them are located in the outer $25^{\prime}-30^{\prime}$ annulus, and none was identified in the $10^{\prime}$-radius central area. These objects are not included in the histogram plots of Figure 8. 


\subsection{Radial surface density profiles}

We studied the radial surface density profiles of $\sigma$ Orionis sources of different mass within the circular area of radius $=30^{\prime}$. To extend the profiles to further distances from the cluster center, we also explored the surrounding regions covered by the VISTA survey (only tile 16, other tiles to the North of the cluster are not as deep as the observations of $\sigma$ Orionis). Within tile 16 (Figure 1), we avoided the regions East of the cluster because of the high extinction associated to the Horsehead Nebula. We selected the area enclosed between $30^{\prime}$ and $50^{\prime}$ West of the $\sigma$ Orionis cluster $\left(1090 \operatorname{arcmin}^{2}\right)$. After applying the $Z J$ photometric criteria described in Section 3.2, a total of 14 sources were identified with $J$ magnitudes in the interval 13-20.5 mag (see Table 11). Based on WISE $12 \mu \mathrm{m}$ data, three of them show infrared flux excesses. We also checked that all these sources follow the cluster sequence in the VISTA color magnitude diagrams of Figure 3, i.e., the 14 objects successfully pass all the photometric criteria for being considered as cluster member candidates, suggesting that the cluster area may extend up to or even beyond a radius of $50^{\prime}$ from the center.

The resulting radial surface density profile of the $210 \sigma$ Orionis member candidates is displayed in Figure 9. In general, there is a large object concentration in the central $10^{\prime}$ and a smooth decay by more than one order of magnitude toward the periphery. Previous studies (Béjar et al. 2004a, 2011; Caballero 2008b; Lodieu et al. 2009) have found distributions similar to ours. The cluster radial surface density profile can be reproduced by an exponential law $\rho=\rho_{0} e^{-\frac{r}{r_{0}}}$ (van den Bergh $\&$ Sher 1960). We found a central density $\rho_{0}=0.4 \operatorname{arcmin}^{-2}\left(\rho_{0} \sim 36 \mathrm{pc}^{-2}\right)$ and a characteristic radius $r_{0} \sim 12^{\prime}(1.2 \mathrm{pc})$. A similar result was reported by Béjar et al. (2011), who found $r_{0} \sim 1.0 \mathrm{pc}$.

The radial surface density profiles for different mass intervals (stars, $J=13-14.5$ mag; brown dwarfs, $J=14.5-18.1 \mathrm{mag}$; and planetary-mass objects, $J>18.1 \mathrm{mag}$ ) up to $50^{\prime}$ from the cluster center are shown in Figure 10. The upper panel displays the profiles normalized to the total number of objects in each magnitude interval, and the lower panel depicts the measured densities without any normalization. Radial density profiles were normalized to the total number of sources rather than to the number of sources in the central cluster region to avoid the large uncertainties introduced by small numbers. In the very central region of the cluster $\left(\leq 5^{\prime}\right)$, we took into account the area lost (about $21 \%$ ) because of the presence of numerous very bright stars preventing us from detecting planetary-mass candidates, and we thus applied an area correction only to the faintest magnitude bins.

The radial distributions of very low mass stars and brown dwarfs do not differ significantly. The radial profile of the planetary-mass object density might deviate from those of very low-mass stars and brown dwarfs at the level of $1.5-\sigma$, since it appears to be flatter in the central $20^{\prime}$. Nevertheless, at farther separations from the cluster center there is an obvious decrease in the surface planetary-mass object density. This feature can be interpreted in favor of the cluster membership of the majority of the planetary mass candidates found in our study, because if they were merely photometric contaminants they would present a flat radial profile independently of the separation from the cluster center, as expected from an homogeneous density of contaminants. 
Nevertheless, we argue that the observed differences between the radial profiles of very low-mass stars, brown dwarfs, and planetary-mass objects are not statistically significant, and that all three radial profiles are consistent with the hypothesis of distributions with a similar origin (after applying the Kolmogorov-Smirnov test ${ }^{5}$, we obtained $P>0.93$ for the comparison of the very low-mass stars-planetary-mass objects samples and the brown dwarfs-planetary-mass objects samples). Furthermore, Bouy et al. (2009) studied the core of the $\sigma$ Orionis cluster using adaptive optics and found a relatively large number of planetary-mass candidates; if confirmed to be true $\sigma$ Orionis members, they would increase the central density of the cluster planetary mass population. This contrasts with the underabundance of substellar objects in the central cluster region reported by Caballero (2007a, 2008b) and Lodieu et al. (2009). In the context of a dynamically evolved system, one would expect the least massive objects to be located far away from the cluster center, but this does not appear to be the case in $\sigma$ Orionis, thus confirming that $\sigma$ Orionis is younger than its relaxation time.

The radial surface density profile of the $Z$-band non-detected sources (Section 3.4) is displayed in the upper panel of Figure 10. In contrast to the very low-mass stars and brown dwarfs of $\sigma$ Orionis, it shows a nearly flat distribution or a moderate density increase at separations larger than $20^{\prime}$ from the cluster center. This feature along with the previously discussed spatial distribution may be ascribed to a faint, very low-mass $\sigma$ Orionis population inhabiting the peripheral regions of the cluster or, more likely, to a population of field contaminants (also see Section 5).

According to previous studies, over $80 \%$ of confirmed cluster members lie within a circular area of radius $=30^{\prime}$ (Béjar et al. 2004a, 2011; Caballero 2008b; Lodieu et al. 2009). Using the VISTA data, we estimate that $\sim 65$ potential cluster members following the cluster photometric sequence in all possible filters could be located at radial separations between $30^{\prime}$ and $50^{\prime}$ from the cluster center (14 candidates were already identified to the West of the cluster uncovering about 1/4.6 of the total coronal area). We thus estimate that the circular survey of radius $=30^{\prime}$ accounts for more than $75 \%$ of the $\sigma$ Orionis low-mass population. Actually, this fraction is expected to be significantly larger because the number of field contaminants increases at a high rate at far separations from the $\sigma$ Orionis center as compared to true cluster members.

By taking the expected number of field dwarf contaminants per mass interval computed in Section 5, we derived the surface density of dwarf contamination in our survey. It is plotted in the bottom panel of Figure 10. Up to $30^{\prime}-35^{\prime}$, the cluster surface densities are higher than the level of expected contamination for all mass intervals (very low-mass stars, brown dwarfs, and planetary-mass objects). At larger separations from the cluster center, only the stellar bin appears overnumerous with respect to the contamination level, while the substellar mass intervals reach nearly the same value as the expected contamination, indicating that (i) large observational efforts

\footnotetext{
${ }^{5}$ In a Kolmogorov-Smirnov test the P-value is an indicator of the amount of evidence against the hypothesis of distributions with the same origin. The smaller the $\mathrm{P}$-value, the stronger the evidence. The reference value is 0.05 typically.
} 
are required to distinguish true cluster members from contaminants and, (ii) any pure photometric statistical study at distances beyond $\sim 35^{\prime}$ from the cluster center has little significance. Therefore, we shall restrict the $\sigma$ Orionis mass function analysis of the next section to the area within a radius of $30^{\prime}$.

\subsection{Objects with and without disks}

Radial surface density profiles of $\sigma$ Orionis candidates with and without flux excesses at $8.0 \mu \mathrm{m}$ are shown in Figure 11. Here, we do not distinguish different mass intervals. We counted objects inside concentric annuli of $5^{\prime}$ in size from the cluster center up to a separation of $25^{\prime}$. The lower panel of Figure 11 depicts our derivations of the disk/infrared flux excesses fractions at different separations from the cluster center. From both panels it can be seen that there is no obvious difference in the distribution or disk fraction of both $\sigma$ Orionis populations; the disk fraction remains nearly constant at the 1- $\sigma$ level all across the explored cluster area. A similar property is observed when considering flux excesses at $4.5 \mu \mathrm{m}$. This result is in agreement with the findings by Oliveira et al. (2006), who concluded that there is no spatial segregation for low-mass objects with and without circum(sub)stellar disks.

\section{The $\sigma$ Orionis mass function}

Our study allowed us to derive a reliable, comprehensive mass function for the $\sigma$ Orionis cluster. First, the VISTA survey homogeneously covers a large extension of the cluster area with detection completeness in the mass interval $0.25-0.006 M_{\odot}$ (at the age of $3 \mathrm{Myr}$ and distance of $352 \mathrm{pc}$ ). Second, we showed that our analysis of a circled area of radius of $30^{\prime}$ around the $\sigma$ Ori $\mathrm{AB}$ star uncovered $>75 \%$ of all cluster members, thus giving statistical weight to the mass function derivation. Third, a large fraction of our VISTA candidates have spectral and/or photometric confirmation of their young age, and thus, they are likely $\sigma$ Orionis members. As stated in Section 3,129 objects out of the total of 210 candidates were confirmed to be young in the literature. Here, we confirmed the presence of infrared flux excesses for an additional amount of 21 objects (15 stars and 6 substellar sources). Therefore, our mass function derivation using the VISTA data relies on a sample of 210 objects, $71 \%$ of which have a confirmed young age fully compatible with cluster membership.

\subsection{Substellar mass spectrum}

Using the VISTA $Z J$ selected cluster candidates in the circular area of radius $=30^{\prime}$ around the cluster center, we built the $\sigma$ Orionis luminosity function (number of objects per $J$-band magnitude interval) depicted in Figure 12. In the range of absolute magnitudes $M_{J}=5-8$ mag, 
the cluster luminosity function steadily declines, while it remains nearly constant from $M_{J}=8$ through 13 mag. The marked drop at $M_{J} \sim 8$ mag corresponds to spectral types mid- to late-M, a feature attributed to the appearance of dust formation in cool atmospheres (Dobbie et al. 2002). Nevertheless, there is no definitive observational or theoretical explanation for it.

Figure 12 also displays the $\sigma$ Orionis low-mass stellar and substellar-mass spectrum (i.e., number of objects per mass interval, $\Delta N / \Delta M$ ) obtained from the cluster luminosity function and the 3 Myr theoretical mass-luminosity relations indicated in Section 4. The stellar, brown dwarf, and planetary-mass regimes were divided into two bins each. We corrected the cluster mass spectrum from contamination by field dwarfs as described in Section 5. In the following, we shall consider only the decontaminated mass spectrum. The "VISTA" mass spectrum is complete in the mass interval $0.25-0.006 M_{\odot}$, where completeness is driven by the $Z$-band observations.

The mass spectrum smoothly increases toward smaller mass. There is a continuous transition from the low-mass stellar domain to the brown dwarf regime, and from here to the planetary-mass objects with no apparent change in the slope of the mass spectrum. It can be fit by a power-law function $\Delta N / \Delta M \propto M^{-\alpha}$, for which we measured an index $\alpha=0.6 \pm 0.2$ in the mass interval 0.25-0.006 $M_{\odot}$. This result is in agreement with the derivations found in the literature (Caballero 2011 and references therein). For example, the first substellar mass spectrum obtained for the mass range $0.2-0.013 M_{\odot}$ and an area of $847 \mathrm{arcmin}^{2}$ by Béjar et al. (2001) was fit with $\alpha=0.8 \pm 0.4$. Caballero et al. (2007) measured $\alpha=0.6 \pm 0.2$ for the mass interval $0.11-0.006 M_{\odot}$ and an area of $790 \operatorname{arcmin}^{2}$. The similarity of our measurement and that of Caballero et al. (2007) is remarkable since both working mass intervals are alike, but our explored area is 3.5 times larger. The recent works by Lodieu et al. (2009) and Béjar et al. (2011) covered an area similar to our VISTA survey, and they found $\alpha=0.5 \pm 0.2\left(0.5-0.01 M_{\odot}\right)$ and $\alpha=0.7 \pm 0.3\left(0.1-0.013 M_{\odot}\right)$, respectively. As discussed in Béjar et al. (2011), the various brown dwarf mass spectrum studies carried out in different star-forming regions and associations with ages below 100-200 Myr have yielded rising functions with typical power-law indices between 0.4 and 1.0, e.g., $\rho$ Ophiuchi (Luhman et al. 2000), IC 348 (Najita et al. 2000; Luhman et al. 2003), the Trapezium (Lucas \& Roche 2000; Hillenbrand \& Carpenter 2000; Luhman et al. 2000), Chamaeleon (López-Martí et al. 2004; Luhman 2007), $\lambda$ Orionis (Barrado y Navascués et al. 2004), Upper Scorpius (Lodieu et al. 2007a), $\alpha$ Persei (Barrado y Navascués et al. 2002a), Blanco 1 (Moraux et al. 2007), and the Pleiades cluster (Moraux et al. 2003; Bihain et al. 2006; Lodieu et al. 2007a).

The extrapolation of the $\sigma$ Orionis mass spectrum between the $Z$-band completeness and the $Z$-band detection limit (or $J=19.95-20.5 \mathrm{mag}$ ) yields about five cluster planetary-mass objects in the range $0.006-0.004 M_{\odot}$ with expected spectral types from mid- to late-L based on their predicted surface temperatures at the age of $3 \mathrm{Myr}$. This number estimate is consistent with our measurement free of contaminants $(7 \pm 2$ objects) at the $1-\sigma$ level (Figure 12). The $\sigma$ Orionis mass function appears to extend from high mass stars down to planetary-mass objects with $0.004 M_{\odot}$. For smaller masses and the magnitude interval $J=20.5-21$ mag (completeness magnitude of the $J$-band data), and based on the theoretical $3 \mathrm{Myr}$ model and the normalized sequence of field L and 
T dwarfs depicted in Figures 2 and 3, the VISTA $J$-band data are sensitive to $\sigma$ Orionis sources warmer than about T6. In our study we identified 3-4 T-type objects: the previously known S Ori 70 (T5.5, Zapatero Osorio et al. 2002a) and S Ori 73 (T4, Bihain et al. 2009; Peña Ramírez et al. 2011), a new object S Ori J053804.65-021352.5 (see Table 8) with optical and infrared colors consistent with an ultracool atmosphere, and one source detected only in the $J$-band (see Table 9 and Section 3.4). The cluster membership of the two former objects was recently discussed by Peña Ramírez et al. (2011), who found that both T dwarfs have proper motions higher $(>4-\sigma)$ than that of the $\sigma$ Orionis cluster. The extrapolation of the $\sigma$ Orionis mass spectrum down to $0.003 M_{\odot}$ or $J=21$ mag yields a population of T sources that clearly outnumbers our findings. Furthermore, the expected contamination of $\sim 2$ field T-type objects (Section 5) contributes to increase the observed discrepancy. There are two possible explanations to account for this difference: (i) the evolutionary models may make wrong predictions particularly at these low masses and/or our conversions from theoretical parameters to observables based on field calibrations are not valid at very low masses

and young ages; (ii) and/or the $\sigma$ Orionis mass spectrum (or mass function) may have a turn over (and possibly a mass cut-off) below $0.004 M_{\odot}$ or $J=20.5 \mathrm{mag}$ (in agreement with Bihain et al. 2009). Deeper images are required for a robust claim on the detection of a low-mass turn over or cut-off for the $\sigma$ Orionis mass function, which has large impact in the theory of substellar formation processes. If none of the 3-4 T-type objects found in the VISTA $\sigma$ Orionis data turned out to be a cluster member, we estimate the field $\mathrm{T}$ dwarf density at factors of 1.5-7.5 higher than the density measurements available in the literature (Burgasser 2007; Metchev et al. 2008; Reylé et al. 2010).

\subsection{From high mass stars to planets}

The mass spectrum presented in the previous section is focused on the low mass stellar and substellar domains of $\sigma$ Orionis. To determine the cluster mass function covering a wider mass range we need to expand our study to more massive cluster members. The Mayrit catalog (Caballero 2008c) presents a compilation of $338 \sigma$ Orionis star and brown dwarf member candidates, 241 (71\%) of which have known features of youth. Interestingly, the area covered by the Mayrit catalog fully overlaps with our VISTA explored region. Therefore, both the Mayrit catalog and our VISTA survey were merged to build the complete $\sigma$ Orionis mass function from the most massive stars $\left(\sim 19 M_{\odot}\right)$ to the planetary-mass objects of $0.006 M_{\odot}$. Our VISTA data and the Mayrit catalog have a common magnitude interval where both surveys are complete: $J=13-14.6$ mag, where the number of detections is quite similar for the two catalogs. For $J<13 \mathrm{mag}$, there are 189 Mayrit sources, which we have added to our 210 VISTA objects. Of the 189 Mayrit sources, 85 (45 $\pm 6 \%$ ) have infrared flux excesses at $8.0 \mu \mathrm{m}$, and more than the $70 \%$ show spectroscopic features of youth or X-ray emission. For both samples, the number of confirmed young objects and likely $\sigma$ Orionis members is similar (71\%) and statistically significant, rendering our cluster mass function reliable.

The combined Mayrit and VISTA J-band luminosity function is shown in Figure 13. The observed differences between the Mayrit catalog and the VISTA data in the overlapping magnitude 
range $J=13-14.6 \mathrm{mag}\left(M_{J}=5.3-6.9 \mathrm{mag}\right)$ are within the Poisson errors. This adds support to the combination of both catalogs.

To build the mass function we used the stellar mass-luminosity relationships corresponding to the $3 \mathrm{Myr}$, solar metallicity isochrones from Siess et al. (2000) and Baraffe et al. (1998) (NextGen model). Theoretical luminosities and effective temperatures were transformed to observable magnitudes and colors using the relations given in Worthey \& Lee (2011). As illustrated in Figure 14, the mass-luminosity relationships used are quite similar for a wide range of masses, and only differ significantly between $\sim 0.1$ and $0.3 M_{\odot}$. Actually, this difference becomes larger for a broader mass interval at ages younger than $3 \mathrm{Myr}$, and both models converge toward older ages (see Figure 14), indicating that the choice of the zero age for the theoretical computations may account for the discrepancies. For the age of $3 \mathrm{Myr}$, the mass derivations obtained from the NextGen and Siess et al. (2000) models do not differ by more than 20\%. The masses of the most luminous $\sigma$ Ori stars ( $\sigma$ Ori Aa, Ab, B, D and E ) were taken from Simón-Díaz et al. (2011). These authors reported the detection of a third massive star component in the $\sigma$ Orionis AB system, traditionally considered to be a binary system. Simón-Díaz et al. (2011) employed a distance of 385 pc to the cluster. We checked that by adopting the distance of $352 \mathrm{pc}$ the most massive bin is not changed in our study.

The combined $\sigma$ Orionis stellar and substellar mass spectrum is depicted in Figure 15 . We plotted the derivations using the NextGen and Siess et al. (2000) models. To give a similar statistical weight to each mass bin, we considered mass intervals with similar amounts of $\sigma$ Orionis member candidates (20-30), thus the mass bin size is not constant along the mass spectrum. Only the incomplete bin in the planetary-mass regime and the complete most massive bin contain less number of objects. We remark the good match between the Mayrit and the VISTA catalogs since there is no obvious discontinuity at the mass both samples were merged $\left(0.25 M_{\odot}\right)$.

The $\sigma$ Orionis mass spectrum is a rising function, i.e., the number of $\sigma$ Orionis members increases toward low masses from $\sim 19$ to $0.006 M_{\odot}$. It can be fit by two power-law equations of different exponents. In Table 12 we summarize the $\alpha$ parameters found for the mass intervals 19-0.35 $M_{\odot}$ and $0.35-0.006 M_{\odot}$. To take into account the differences that may arise from the two employed theoretical mass-luminosity relations, we also provide fits for the following cases: case I where the Lyon- and Siess et al. (2000)-based masses cover the intervals 0.006-1 $M_{\odot}$ and 1-19 $M_{\odot}$, respectively, and case II where the Siess et al. (2000)-based masses cover 0.3-19 $M_{\odot}$ and the smaller masses are derived using the Lyon isochrone. The fits shown in Figure 15 correspond only to case I (for the clarity of the Figure). Despite the differences in the models (e.g., from Figure 15 it can be seen that some mass bins differ by more than the Poisson uncertainty), the derived $\alpha$ values are consistent with each other at the 1- $\sigma$ level. The $\alpha$ exponent obtained for the mass range 19-0.35 $M_{\odot}$ ( $\alpha \sim 1.7)$ appears to be slightly smaller than the Salpeter value of 2.35 (Salpeter 1955).

Figure 15 also illustrates the comparison of the $\sigma$ Orionis mass spectrum with the multiple segment power-law function proposed by Kroupa (2001). This author defined an average or Galactic-field mass spectrum with changes in the power-law index at two masses: $\sim 0.5 M_{\odot}$ and 
$\sim 0.08 M_{\odot}$. The Kroupa functional form normalized to the total number of $\sigma$ Orionis objects is shown in Figure 15. According to this function, the number of substellar objects relative to the population of stars appears to be larger than what is observed from our $\sigma$ Orionis mass spectrum. However, we remark that the $\sigma$ Orionis mass spectrum and Kroupa (2001) function are consistent within the uncertainties quoted for both. We found that a two segment power-law function $\left(\alpha=1.7 \pm 0.2\right.$ for $M>0.35 M_{\odot}$, and $\alpha=0.6 \pm 0.2$ for $\left.M<0.35 M_{\odot}\right)$ provides a better fit to the cluster data.

To compare the $\sigma$ Orionis mass spectrum with known mass functions in other star forming regions and the field, we built the cluster mass function as originally defined by Salpeter (1955), i.e., $\xi(\log M)=\Delta N / \Delta \log M$. It is displayed in Figure 15. We performed a lognormal fit to the data by finding the characteristic mass $\left(M_{c}\right.$, the mass at which the mass function reaches its maximum) and the variance $(\sigma)$ of the distribution:

$$
\xi(\log M) \sim \exp \left(-\frac{\left(\log M-\log M_{c}\right)^{2}}{2 \sigma^{2}}\right)
$$

The derived parameters for cases I and II are listed in Table 12. The characteristic mass was found at $0.27 M_{\odot}$ for both cases, the variance of the functions ranged from 0.6 to 0.7 . This is in agreement with other works on open clusters, e.g., Pleiades (Moraux et al. 2003), $\rho$ Ophiuchi, IC 348 and the Trapezium (Luhman et al. 2000), Chamaeleon I (Luhman 2007), and the Galactic field (Chabrier 2005).

In Figure 15, we also compared the $\sigma$ Orionis mass function with the functional form of Chabrier (2005), the latter being normalized to the total number of $\sigma$ Orionis sources. Chabrier (2005) function consists of a combined Salpeter's power-law for $M>1 M_{\odot}$ and a lognormal parametrization for lower masses, where $M_{c}=0.25 M_{\odot}$ and variance $\sigma=0.55$. The similarity between this functional form and the pure lognormal one is noteworthy for the stellar mass regime (see Table 12). However, the $\sigma$ Orionis planetary-mass interval clearly outnumbers the prediction of the Chabrier (2005) function. This author did not cover such low masses in his study. By adopting Chabrier (2005) functional form and fitting the characteristic mass and variance of the lognormal equation from 1 down to $0.006 M_{\odot}$, we found $M_{c} \sim 0.25 M_{\odot}$ and $\sigma=0.5-0.6$ for $\sigma$ Orionis; the planetary-mass bins are still not well reproduced. As for the cluster mass spectrum, we conclude that two power-law expressions appear to fit better the $\sigma$ Orionis mass function, from the very high masses down to the free-floating planetary-mass objects; these power-laws are illustrated in Figure 15.

Sumi et al. (2011) reported the discovery of a large population of unbound planetary mass objects located at wide separations from their parent stars by the gravitational microlensing technique. In their work, the authors claimed that the number of brown dwarfs and planetary mass objects derived from microlensing events is $\sim 0.7$ and $\sim 1.8$ times the number of main sequence stars with masses in the interval $0.08-1.0 M_{\odot}$. Our results for the $\sigma$ Orionis cluster indicate that brown dwarfs represent about one third of the population of sub-solar mass stars $\left(0.08-1.0 M_{\odot}\right)$, while 
planetary mass objects down to $\sim 6 M_{\text {Jup }}$ represent a fraction of only $\sim 0.2$. Our study found a relative number of substellar objects smaller than that obtained by Sumi et al. (2011). However, the alternative model presented by the authors, where they used a discontinuous substellar mass function (model 3 from Sumi et al. 2011), suggests a fraction of brown dwarfs relative to the number of stars less massive than the Sun of $\sim 0.2$, which is consistent with our results. Because our search is complete down to $\sim 6 M_{\text {Jup }}$, objects of $1 M_{\text {Jup }}$ are not covered by the VISTA data, then we cannot compare directly to the results of Sumi et al. (2011) for this mass regime.

\section{Conclusions}

Using VISTA Orion data obtained in the $Z Y J H K_{s}$ filters, we performed a photometric exploration of a circular area of radius $30^{\prime}$ around the multiple, O-type star $\sigma$ Ori. The survey uncovered $>75 \%$ of the $\sigma$ Orionis cluster population with masses ranging from $0.25 M_{\odot}(J=$ $13 \mathrm{mag})$ to $0.006 M_{\odot}(Z$-band completeness at $22.6 \mathrm{mag})$ and $0.003 M_{\odot}(J$-band completeness at $21 \mathrm{mag})$. These mass estimates are based on theoretical isochrones of $3 \mathrm{Myr}$, solar metallicity, and shifted to the distance of $352 \mathrm{pc}$, which are the canonical parameters of the $\sigma$ Orionis cluster. The VISTA data were combined with optical ( $I$-band) and mid-infrared WISE and Spitzer photometry to identify a total of $210 \sigma$ Orionis candidates detected at both $Z$ - and $J$-passbands that comply with all photometric criteria expected for bona-fide cluster members. Of the 210, 23 are new discoveries with mass estimates in the planetary-mass interval $0.011-0.004 M_{\odot}$ and likely $\mathrm{L}$ spectral types. One of them, S Ori J053804.65-021352.5, has VISTA and Spitzer colors compatible with a "methane" spectral type T0-T4. Our findings double the number of $\sigma$ Orionis planetary-mass candidates known to date.

Based on infrared flux excesses at $8.0 \mu \mathrm{m}$, we derived a disk frequency of $41 \pm 8 \%$ and $39 \pm 9 \%$ for very low-mass stars $\left(0.25-0.072 M_{\odot}\right)$ and brown dwarfs $\left(0.072-0.012 M_{\odot}\right)$. As for the planetary domain $\left(0.012-0.004 M_{\odot}\right)$, the study of the presence of infrared flux excesses was based on the deeper Spitzer $4.5 \mu \mathrm{m}$ data, from which we measured that $\geq 31 \pm 11 \%$ of the $\sigma$ Orionis planetary-mass objects harbor warm inner disks.

The combination of an homogeneous survey depth, wide spatial and mass coverages, and high percentage of sources with confirmed membership in $\sigma$ Orionis $(\sim 70 \%)$ has allowed us to confidently study the cluster spatial distribution and mass function. Low-mass stars, brown dwarfs, and planetary-mass objects in $\sigma$ Orionis are spatially concentrated with an effective radius of $12^{\prime}$ (1.2 pc) around the multiple star $\sigma$ Ori. They have a central density of $\rho_{0} \sim 0.4 \operatorname{arcmin}^{-2}\left(36 \mathrm{pc}^{-2}\right)$. The radial density distributions of all three mass intervals are alike, suggesting little dynamical evolution of the cluster. Furthermore, we find no significant evidence for spatial distribution differences between the disk-bearing and diskless cluster members, suggesting that there is no spatial segregation between these two type of populations.

The $\sigma$ Orionis low-mass spectrum is a smooth rising function that can be fit with a single 
power-law expression $\left(\Delta N / \Delta M \sim M^{-\alpha}\right)$, where $\alpha=0.6 \pm 0.2$ for the mass interval $0.25-0.006 M_{\odot}$ ( $Z$-band completeness or $J=20 \mathrm{mag}$ ). The $\sigma$ Orionis mass spectrum extends well into the planetarymass regime down to $0.004 M_{\odot}$. Using the Mayrit catalog by Caballero (2008c), we were able to expand the $\sigma$ Orionis mass function and the mass spectrum up to the most massive, O-type stars of the cluster, thus covering the wide mass range $\sim 19-0.006 M_{\odot}$. We compared the $\sigma$ Orionis mass function to various functional forms discussed in the literature finding a characteristic mass of $0.27 \pm 0.10 M_{\odot}$ and a variance ranging from 0.5 to 0.7 in the case of a lognormal mass function. However, this functional form appears to underestimate the number of cluster planetary-mass objects. The entire $\sigma$ Orionis mass function and mass spectrum are reasonably described by two power-law expressions with mass spectrum indices of $\alpha=1.7 \pm 0.2$ for $M>0.35 M_{\odot}$, and $\alpha=0.6 \pm 0.2$ for $M<0.35 M_{\odot}$. The low number of T-type objects found in the VISTA $\sigma$ Orionis survey (two previously known ones, S Ori 70 and 73, and a new one reported here) as compared to the prediction made by the extrapolation of the $\sigma$ Orionis mass function between 0.004 and $0.003 M_{\odot}$ (or between $J=20.5 \mathrm{mag}$ and $J$-band completeness) seems to indicate that either the cluster substellar mass function has a turnover (and possibly a mass cut-off) at around $0.004 M_{\odot}$, field $\mathrm{T}$ dwarf densities are underestimated in the literature, and/or cluster T-type objects are fainter than predicted by theoretical models.

We thank the referee, José A. Caballero, for constructive comments. Based on observations made with ESO Telescopes at Cerro Paranal Observatory during VISTA science verification, under the program ID 60.A-9285(B). This work is in part based on observations made with the Spitzer Space Telescope, which is operated by the Jet Propulsion Laboratory, California Institute of Technology under a contract with NASA. This publication makes use of data products from the Wide-field Infrared Survey Explorer, which is a joint project of the University of California, Los Angeles, and the Jet Propulsion Laboratory/California Institute of Technology, funded by the National Aeronautics and Space Administration. IRAF is distributed by National Optical Astronomy Observatory, which is operated by the Association of Universities for Research in Astronomy, Inc., under contract with the National Science Foundation. This research has been supported by the Spanish Ministry of Economics and Competitiveness under the projects AYA2010-21308-C3-02 and AYA2010-20535. 


\section{REFERENCES}

Andrews, S. M., Reipurth, B., Bally, J., \& Heathcote, S. R. 2004, ApJ, 606, 353

Arnaboldi, M., Petr-Gotzens, M., Rejkuba, M., Neeser, M., Szeifert, T., Ivanov, V. D., Hummel, W., Hilker, M., Neumayer, N., Møller, P., Nilsson, K., Venemans, B., Hatziminaoglou, E., Hussain, G., Stanke, T., Teixeira, P., Ramsay, S., Retzlaff, J., Slijkhuis, R., Comerón, F., Melnick, J., Romaniello, M., Emerson, J., Sutherland, W., Irwin, M., Lewis, J., Hodgkin, S., \& Gonzales-Solares, E. 2010, The Messenger, 139, 6

Baraffe, I., Chabrier, G., Allard, F., \& Hauschildt, P. H. 1998, A\&A, 337, 403

Baraffe, I., Chabrier, G., Barman, T. S., Allard, F., \& Hauschildt, P. H. 2003, A\&A, 402, 701

Barrado y Navascués, D., Béjar, V. J. S., Mundt, R., Martín, E. L., Rebolo, R., Zapatero Osorio, M. R., \& Bailer-Jones, C. A. L. 2003, A\&A, 404, 171

Barrado y Navascués, D., Bouvier, J., Stauffer, J. R., Lodieu, N., \& McCaughrean, M. J. 2002a, A\&A, 395, 813

Barrado y Navascués, D., Stauffer, J. R., Bouvier, J., Jayawardhana, R., \& Cuillandre, J.-C. 2004, ApJ, 610, 1064

Barrado y Navascués, D., Zapatero Osorio, M. R., Béjar, V. J. S., Rebolo, R., Martín, E. L., Mundt, R., \& Bailer-Jones, C. A. L. 2001, A\&A, 377, L9

Barrado y Navascués, D., Zapatero Osorio, M. R., Martín, E. L., Béjar, V. J. S., Rebolo, R., \& Mundt, R. 2002b, A\&A, 393, L85

Bastian, N., Covey, K. R., \& Meyer, M. R. 2010, ARA\&A, 48, 339

Béjar, V. J. S. 2001, PhD thesis, Instituto de Astrofísica de Canarias

Béjar, V. J. S., Caballero, J. A., Rebolo, R., Zapatero Osorio, M. R., \& Barrado y Navascués, D. 2004a, Ap\&SS, 292, 339

Béjar, V. J. S., Martín, E. L., Zapatero Osorio, M. R., Rebolo, R., Barrado y Navascués, D., Bailer-Jones, C. A. L., Mundt, R., Baraffe, I., Chabrier, C., \& Allard, F. 2001, ApJ, 556, 830

Béjar, V. J. S., Zapatero Osorio, M. R., \& Rebolo, R. 1999, ApJ, 521, 671

-. 2004b, AN, 325, 705

Béjar, V. J. S., Zapatero Osorio, M. R., Rebolo, R., Caballero, J. A., Barrado, D., Martín, E. L., Mundt, R., \& Bailer-Jones, C. A. L. 2011, ApJ, 743, 64 
Bihain, G., Rebolo, R., Béjar, V. J. S., Caballero, J. A., Bailer-Jones, C. A. L., Mundt, R., AcostaPulido, J. A., \& Manchado Torres, A. 2006, A\&A, 458, 805

Bihain, G., Rebolo, R., Zapatero Osorio, M. R., Béjar, V. J. S., Villó-Pérez, I., Díaz-Sánchez, A., Pérez-Garrido, A., Caballero, J. A., Bailer-Jones, C. A. L., Barrado y Navascués, D., Eislöffel, J., Forveille, T., Goldman, B., Henning, T., Martín, E. L., \& Mundt, R. 2009, A\&A, 506, 1169

Bouvier, J., Stauffer, J. R., Martín, E. L., Barrado y Navascués, D., Wallace, B., \& Béjar, V. J. S. 1998, A\&A, 336, 490

Bouy, H., Huélamo, N., Martín, E. L., Marchis, F., Barrado Y Navascués, D., Kolb, J., Marchetti, E., Petr-Gotzens, M. G., Sterzik, M., Ivanov, V. D., Köhler, R., \& Nürnberger, D. 2009, A\&A, 493, 931

Brown, A. G. A., de Geus, E. J., \& de Zeeuw, P. T. 1994, A\&A, 289, 101

Burgasser, A. J. 2007, ApJ, 659, 655

Burgasser, A. J., Kirkpatrick, J. D., McGovern, M. R., McLean, I. S., Prato, L., \& Reid, I. N. 2004, ApJ, 604, 827

Burningham, B., Naylor, T., Littlefair, S. P., \& Jeffries, R. D. 2005, MNRAS, 356, 1583

Burningham, B., Pinfield, D. J., Lucas, P. W., Leggett, S. K., Deacon, N. R., Tamura, M., Tinney, C. G., Lodieu, N., Zhang, Z. H., Huélamo, N., Jones, H. R. A., Murray, D. N., Mortlock, D. J., Patel, M., Barrado y Navascués, D., Zapatero Osorio, M. R., Ishii, M., Kuzuhara, M., \& Smart, R. L. 2010, MNRAS, 406, 1885

Caballero, J. A. 2006, PhD thesis, Instituto de Astrofísica de Canarias

-. 2007a, AN, 328, 917

—. 2007b, A\&A, 466, 917

-. 2008a, MNRAS, 383, 750

一. 2008b, MNRAS, 383, 375

-. 2008c, A\&A, 478, 667

Caballero, J. A. 2011, in Stellar Clusters \& Associations: A RIA Workshop on Gaia, 108

Caballero, J. A., Béjar, V. J. S., Rebolo, R., Eislöffel, J., Zapatero Osorio, M. R., Mundt, R., Barrado Y Navascués, D., Bihain, G., Bailer-Jones, C. A. L., Forveille, T., \& Martín, E. L. 2007, A\&A, 470, 903

Caballero, J. A., Béjar, V. J. S., Rebolo, R., \& Zapatero Osorio, M. R. 2004, A\&A, 424, 857 
Caballero, J. A., Burgasser, A. J., \& Klement, R. 2008a, A\&A, 488, 181

Caballero, J. A., Martín, E. L., Dobbie, P. D., \& Barrado Y Navascués, D. 2006, A\&A, 460, 635

Caballero, J. A., Valdivielso, L., Martín, E. L., Montes, D., Pascual, S., \& Pérez-González, P. G. 2008b, A\&A, 491, 515

Casali, M., Adamson, A., Alves de Oliveira, C., Almaini, O., Burch, K., Chuter, T., Elliot, J., Folger, M., Foucaud, S., Hambly, N., Hastie, M., Henry, D., Hirst, P., Irwin, M., Ives, D., Lawrence, A., Laidlaw, K., Lee, D., Lewis, J., Lunney, D., McLay, S., Montgomery, D., Pickup, A., Read, M., Rees, N., Robson, I., Sekiguchi, K., Vick, A., Warren, S., \& Woodward, B. 2007, A\&A, 467, 777

Chabrier, G. 2005, in Astrophysics and Space Science Library, Vol. 327, The Initial Mass Function 50 Years Later, eds. E. Corbelli, F. Palla, \& H. Zinnecker, 41

Chabrier, G., Baraffe, I., Allard, F., \& Hauschildt, P. 2000, ApJ, 542, 464

Dalton, G. B., Caldwell, M., Ward, A. K., Whalley, M. S., Woodhouse, G., Edeson, R. L., Clark, P., Beard, S. M., Gallie, A. M., Todd, S. P., Strachan, J. M. D., Bezawada, N. N., Sutherland, W. J., \& Emerson, J. P. 2006, in Society of Photo-Optical Instrumentation Engineers (SPIE) Conference, Vol. 6269, Society of Photo-Optical Instrumentation Engineers (SPIE) Conference Series

de Wit, W. J., Bouvier, J., Palla, F., Cuillandre, J.-C., James, D. J., Kendall, T. R., Lodieu, N., McCaughrean, M. J., Moraux, E., Randich, S., \& Testi, L. 2006, A\&A, 448, 189

Dobbie, P. D., Pinfield, D. J., Jameson, R. F., \& Hodgkin, S. T. 2002, MNRAS, 335, L79

Emerson, J., McPherson, A., \& Sutherland, W. 2006, The Messenger, 126, 41

Fazio, G. G., Hora, J. L., Allen, L. E., Ashby, M. L. N., Barmby, P., Deutsch, L. K., Huang, J.-S., Kleiner, S., Marengo, M., Megeath, S. T., Melnick, G. J., Pahre, M. A., Patten, B. M., Polizotti, J., Smith, H. A., Taylor, R. S., Wang, Z., Willner, S. P., Hoffmann, W. F., Pipher, J. L., Forrest, W. J., McMurty, C. W., McCreight, C. R., McKelvey, M. E., McMurray, R. E., Koch, D. G., Moseley, S. H., Arendt, R. G., Mentzell, J. E., Marx, C. T., Losch, P., Mayman, P., Eichhorn, W., Krebs, D., Jhabvala, M., Gezari, D. Y., Fixsen, D. J., Flores, J., Shakoorzadeh, K., Jungo, R., Hakun, C., Workman, L., Karpati, G., Kichak, R., Whitley, R., Mann, S., Tollestrup, E. V., Eisenhardt, P., Stern, D., Gorjian, V., Bhattacharya, B., Carey, S., Nelson, B. O., Glaccum, W. J., Lacy, M., Lowrance, P. J., Laine, S., Reach, W. T., Stauffer, J. A., Surace, J. A., Wilson, G., Wright, E. L., Hoffman, A., Domingo, G., \& Cohen, M. 2004, ApJS, 154, 10

Franciosini, E., Pallavicini, R., \& Sanz-Forcada, J. 2006, A\&A, 446, 501

Garrison, R. F. 1967, PASP, 79, 433 
Golimowski, D. A., Leggett, S. K., Marley, M. S., Fan, X., Geballe, T. R., Knapp, G. R., Vrba, F. J., Henden, A. A., Luginbuhl, C. B., Guetter, H. H., Munn, J. A., Canzian, B., Zheng, W., Tsvetanov, Z. I., Chiu, K., Glazebrook, K., Hoversten, E. A., Schneider, D. P., \& Brinkmann, J. 2004, AJ, 127, 3516

González-García, B. M., Zapatero Osorio, M. R., Béjar, V. J. S., Bihain, G., Barrado y Navascués, D., Caballero, J. A., \& Morales-Calderón, M. 2006, A\&A, 460, 799

González Hernández, J. I., Caballero, J. A., Rebolo, R., Béjar, V. J. S., Barrado y Navascués, D., Martín, E. L., \& Zapatero Osorio, M. R. 2008, A\&A, 490, 1135

Grazian, A., Fontana, A., de Santis, C., Nonino, M., Salimbeni, S., Giallongo, E., Cristiani, S., Gallozzi, S., \& Vanzella, E. 2006, A\&A, 449, 951

Haisch, Jr., K. E., Lada, E. A., \& Lada, C. J. 2001, ApJ, 553, L153

Haro, G. \& Moreno, A. 1953, Boletín de los Observatorios Tonantzintla y Tacubaya, 1, 11

Hernández, J., Hartmann, L., Megeath, T., Gutermuth, R., Muzerolle, J., Calvet, N., Vivas, A. K., Briceño, C., Allen, L., Stauffer, J., Young, E., \& Fazio, G. 2007, ApJ, 662, 1067

Hewett, P. C., Warren, S. J., Leggett, S. K., \& Hodgkin, S. T. 2006, MNRAS, 367, 454

Hillenbrand, L. A. \& Carpenter, J. M. 2000, ApJ, 540, 236

Jayawardhana, R., Ardila, D. R., Stelzer, B., \& Haisch, Jr., K. E. 2003, AJ, 126, 1515

Jeffries, R. D., Maxted, P. F. L., Oliveira, J. M., \& Naylor, T. 2006, MNRAS, 371, L6

Kenyon, M. J., Jeffries, R. D., Naylor, T., Oliveira, J. M., \& Maxted, P. F. L. 2005, MNRAS, 356, 89

Kirkpatrick, J. D., McGraw, J. T., Hess, T. R., Liebert, J., \& McCarthy, Jr., D. W. 1994, ApJS, 94,749

Kroupa, P. 2001, MNRAS, 322, 231

Lee, T. A. 1968, ApJ, 152, 913

Leggett, S. K., Marley, M. S., Freedman, R., Saumon, D., Liu, M. C., Geballe, T. R., Golimowski, D. A., \& Stephens, D. C. 2007, ApJ, 667, 537

Lodieu, N., Burningham, B., Hambly, N. C., \& Pinfield, D. J. 2009, MNRAS, 397, 258

Lodieu, N., de Wit, W.-J., Carraro, G., Moraux, E., Bouvier, J., \& Hambly, N. C. 2011, A\&A, 532, A103 
Lodieu, N., Dobbie, P. D., Deacon, N. R., Hodgkin, S. T., Hambly, N. C., \& Jameson, R. F. 2007a, MNRAS, 380, 712

Lodieu, N., Hambly, N. C., Jameson, R. F., Hodgkin, S. T., Carraro, G., \& Kendall, T. R. 2007b, MNRAS, 374, 372

López-Martí, B., Eislöffel, J., Scholz, A., \& Mundt, R. 2004, A\&A, 416, 555

Lucas, P. W. \& Roche, P. F. 2000, MNRAS, 314, 858

Luhman, K. L. 2007, ApJS, 173, 104

Luhman, K. L., Hernández, J., Downes, J. J., Hartmann, L., \& Briceño, C. 2008, ApJ, 688, 362

Luhman, K. L., Rieke, G. H., Young, E. T., Cotera, A. S., Chen, H., Rieke, M. J., Schneider, G., \& Thompson, R. I. 2000, ApJ, 540, 1016

Luhman, K. L., Stauffer, J. R., Muench, A. A., Rieke, G. H., Lada, E. A., Bouvier, J., \& Lada, C. J. 2003, ApJ, 593, 1093

Luhman, K. L., Whitney, B. A., Meade, M. R., Babler, B. L., Indebetouw, R., Bracker, S., \& Churchwell, E. B. 2006, ApJ, 647, 1180

Lyngå, G. 1981, Astronomical Data Center Bulletin, 1, 90

Lyngå, G. 1983, in Star Clusters and Associations and their Relation to the Evolution of the Galaxy, ed. J. Ruprecht \& J. Palous, 13-27

Martín, E. L., Brandner, W., Bouvier, J., Luhman, K. L., Stauffer, J., Basri, G., Zapatero Osorio, M. R., \& Barrado y Navascués, D. 2000, ApJ, 543, 299

Martín, E. L., Zapatero Osorio, M. R., Barrado y Navascués, D., Béjar, V. J. S., \& Rebolo, R. 2001, ApJ, 558, L117

Maxted, P. F. L., Jeffries, R. D., Oliveira, J. M., Naylor, T., \& Jackson, R. J. 2008, MNRAS, 385, 2210

Mayne, N. J. \& Naylor, T. 2008, MNRAS, 386, 261

McGovern, M. R., Kirkpatrick, J. D., McLean, I. S., Burgasser, A. J., Prato, L., \& Lowrance, P. J. 2004, ApJ, 600, 1020

Metchev, S. A., Kirkpatrick, J. D., Berriman, G. B., \& Looper, D. 2008, ApJ, 676, 1281

Moraux, E., Bouvier, J., Stauffer, J. R., Barrado y Navascués, D., \& Cuillandre, J.-C. 2007, A\&A, 471,499

Moraux, E., Bouvier, J., Stauffer, J. R., \& Cuillandre, J.-C. 2003, A\&A, 400, 891 
Muench, A. A., Lada, E. A., Lada, C. J., \& Alves, J. 2002, ApJ, 573, 366

Najita, J. R., Tiede, G. P., \& Carr, J. S. 2000, ApJ, 541, 977

Oliveira, J. M., Jeffries, R. D., van Loon, J. T., \& Rushton, M. T. 2006, MNRAS, 369, 272

Patten, B. M., Stauffer, J. R., Burrows, A., Marengo, M., Hora, J. L., Luhman, K. L., Sonnett, S. M., Henry, T. J., Raghavan, D., Megeath, S. T., Liebert, J., \& Fazio, G. G. 2006, ApJ, 651,502

Peña Ramírez, K., Zapatero Osorio, M. R., Béjar, V. J. S., Rebolo, R., \& Bihain, G. 2011, A\&A, 532, A 42

Perryman, M. A. C., Lindegren, L., Kovalevsky, J., Hoeg, E., Bastian, U., Bernacca, P. L., Crézé, M., Donati, F., Grenon, M., van Leeuwen, F., van der Marel, H., Mignard, F., Murray, C. A., Le Poole, R. S., Schrijver, H., Turon, C., Arenou, F., Froeschlé, M., \& Petersen, C. S. 1997, A\&A, 323, L49

Petr-Gotzens, M., Alcalá, J. M., Briceño, C., González-Solares, E., Spezzi, L., Teixeira, P., Zapatero Osorio, M. R., Comerón, F., Emerson, J., Hodgkin, S., Hussain, G., McCaughrean, M., Melnick, J., Oliveira, J., Ramsay, S., Stanke, T., Winston, E., \& Zinnecker, H. 2011, The Messenger, 145, 29

Reipurth, B., Bally, J., Fesen, R. A., \& Devine, D. 1998, Nature, 396, 343

Reylé, C., Delorme, P., Willott, C. J., Albert, L., Delfosse, X., Forveille, T., Artigau, E., Malo, L., Hill, G. J., \& Doyon, R. 2010, A\&A, 522, A112

Rigliaco, E., Natta, A., Randich, S., \& Sacco, G. 2009, A\&A, 495, L13

Sacco, G. G., Franciosini, E., Randich, S., \& Pallavicini, R. 2008, A\&A, 488, 167

Sacco, G. G., Randich, S., Franciosini, E., Pallavicini, R., \& Palla, F. 2007, A\&A, 462, L23

Salpeter, E. E. 1955, ApJ, 121, 161

Santini, P., Fontana, A., Grazian, A., Salimbeni, S., Fiore, F., Fontanot, F., Boutsia, K., Castellano, M., Cristiani, S., de Santis, C., Gallozzi, S., Giallongo, E., Menci, N., Nonino, M., Paris, D., Pentericci, L., \& Vanzella, E. 2009, A\&A, 504, 751

Scholz, A. \& Eislöffel, J. 2004, A\&A, 419, 249

Scholz, A. \& Jayawardhana, R. 2008, ApJ, 672, L49

Sherry, W. H., Walter, F. M., \& Wolk, S. J. 2004, AJ, 128, 2316

Sherry, W. H., Walter, F. M., Wolk, S. J., \& Adams, N. R. 2008, AJ, 135, 1616 
Siess, L., Dufour, E., \& Forestini, M. 2000, A\&A, 358, 593

Simón-Díaz, S., Caballero, J. A., \& Lorenzo, J. 2011, ApJ, 742, 55

Skrutskie, M. F., Cutri, R. M., Stiening, R., Weinberg, M. D., Schneider, S., Carpenter, J. M., Beichman, C., Capps, R., Chester, T., Elias, J., Huchra, J., Liebert, J., Lonsdale, C., Monet, D. G., Price, S., Seitzer, P., Jarrett, T., Kirkpatrick, J. D., Gizis, J. E., Howard, E., Evans, T., Fowler, J., Fullmer, L., Hurt, R., Light, R., Kopan, E. L., Marsh, K. A., McCallon, H. L., Tam, R., Van Dyk, S., \& Wheelock, S. 2006, AJ, 131, 1163

Sumi, T., Kamiya, K., Bennett, D. P., Bond, I. A., Abe, F., Botzler, C. S., Fukui, A., Furusawa, K., Hearnshaw, J. B., Itow, Y., Kilmartin, P. M., Korpela, A., Lin, W., Ling, C. H., Masuda, K., Matsubara, Y., Miyake, N., Motomura, M., Muraki, Y., Nagaya, M., Nakamura, S., Ohnishi, K., Okumura, T., Perrott, Y. C., Rattenbury, N., Saito, T., Sako, T., Sullivan, D. J., Sweatman, W. L., Tristram, P. J., Udalski, A., Szymański, M. K., Kubiak, M., Pietrzyński, G., Poleski, R., Soszyński, I., Wyrzykowski, Ł., Ulaczyk, K., \& Microlensing Observations in Astrophysics (MOA) Collaboration. 2011, Nature, 473, 349

van den Bergh, S. \& Sher, D. 1960, Publications of the David Dunlap Observatory, 2, 203

Walter, F. M., Wolk, S. J., Freyberg, M., \& Schmitt, J. H. M. M. 1997, Mem. Soc. Astron. Italiana, 68,1081

Weaver, W. B. \& Babcock, A. 2004, PASP, 116, 1035

Wiramihardja, S. D., Kogure, T., Yoshida, S., Nakano, M., Ogura, K., \& Iwata, T. 1991, PASJ, 43,27

Wiramihardja, S. D., Kogure, T., Yoshida, S., Ogura, K., \& Nakano, M. 1989, PASJ, 41, 155

Wolk, S. J. 1996, PhD thesis, SUNY Stony Brook, New York, USA

Worthey, G. \& Lee, H.-c. 2011, ApJS, 193, 1

Wright, E. L., Eisenhardt, P. R. M., Mainzer, A. K., Ressler, M. E., Cutri, R. M., Jarrett, T., Kirkpatrick, J. D., Padgett, D., McMillan, R. S., Skrutskie, M., Stanford, S. A., Cohen, M., Walker, R. G., Mather, J. C., Leisawitz, D., Gautier, III, T. N., McLean, I., Benford, D., Lonsdale, C. J., Blain, A., Méndez, B., Irace, W. R., Duval, V., Liu, F., Royer, D., Heinrichsen, I., Howard, J., Shannon, M., Kendall, M., Walsh, A. L., Larsen, M., Cardon, J. G., Schick, S., Schwalm, M., Abid, M., Fabinsky, B., Naes, L., \& Tsai, C.-W. 2010, AJ, 140,1868

Zapatero Osorio, M. R., Béjar, V. J. S., Bihain, G., Martín, E. L., Rebolo, R., Villó-Pérez, I., Díaz-Sánchez, A., Pérez Garrido, A., Caballero, J. A., Henning, T., Mundt, R., Barrado y Navascués, D., \& Bailer-Jones, C. A. L. 2008, A\&A, 477, 895 
Zapatero Osorio, M. R., Béjar, V. J. S., Martín, E. L., Rebolo, R., Barrado y Navascués, D., Bailer-Jones, C. A. L., \& Mundt, R. 2000, Science, 290, 103

Zapatero Osorio, M. R., Béjar, V. J. S., Martín, E. L., Rebolo, R., Barrado y Navascués, D., Mundt, R., Eislöffel, J., \& Caballero, J. A. 2002a, ApJ, 578, 536

Zapatero Osorio, M. R., Béjar, V. J. S., Pavlenko, Y., Rebolo, R., Allende Prieto, C., Martín, E. L., \& García López, R. J. 2002b, A\&A, 384, 937

Zapatero Osorio, M. R., Béjar, V. J. S., Rebolo, R., Martín, E. L., \& Basri, G. 1999, ApJ, 524, L115

Zapatero Osorio, M. R., Caballero, J. A., Béjar, V. J. S., Rebolo, R., Barrado Y Navascués, D., Bihain, G., Eislöffel, J., Martín, E. L., Bailer-Jones, C. A. L., Mundt, R., Forveille, T., \& Bouy, H. 2007, A\&A, 472, L9

Zapatero Osorio, M. R., Rebolo, R., Martín, E. L., Basri, G., Magazzù, A., Hodgkin, S. T., Jameson, R. F., \& Cossburn, M. R. 1997, ApJ, 491, L81 


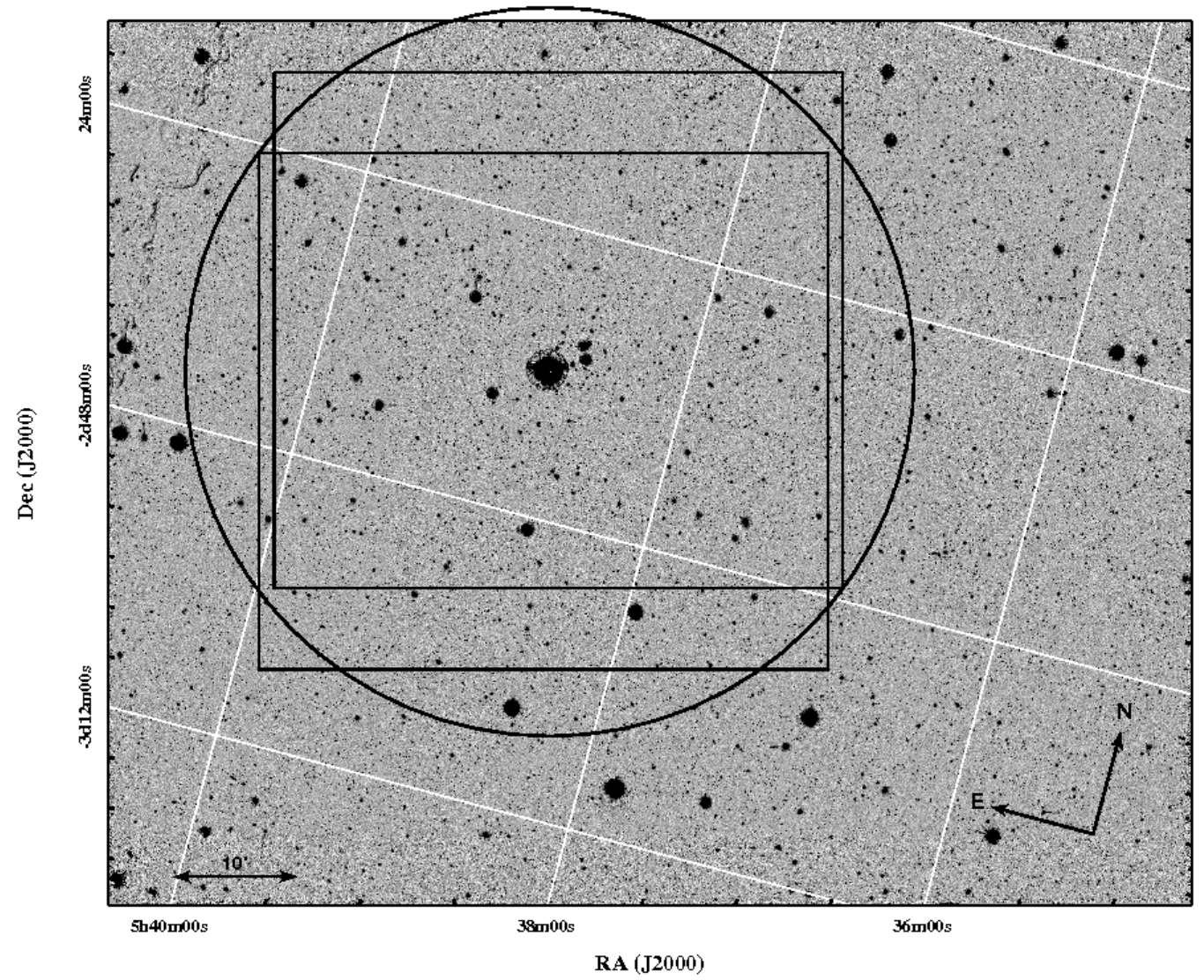

Fig. 1.- Mosaiced VISTA $J$-band image of size $1.2 \times 1.5 \mathrm{deg}^{2}$ (designated as tile no. 16 in the VISTA Orion survey). Our search has explored the region inside the $30^{\prime}$-radius circle centered on the bright, massive multiple system $\sigma$ Ori. The regions explored by Spitzer are shown with squares, the top square corresponds to the [3.6]- and [5.8]-band images, and the bottom square to the [4.5]and [8.0]-band images. 


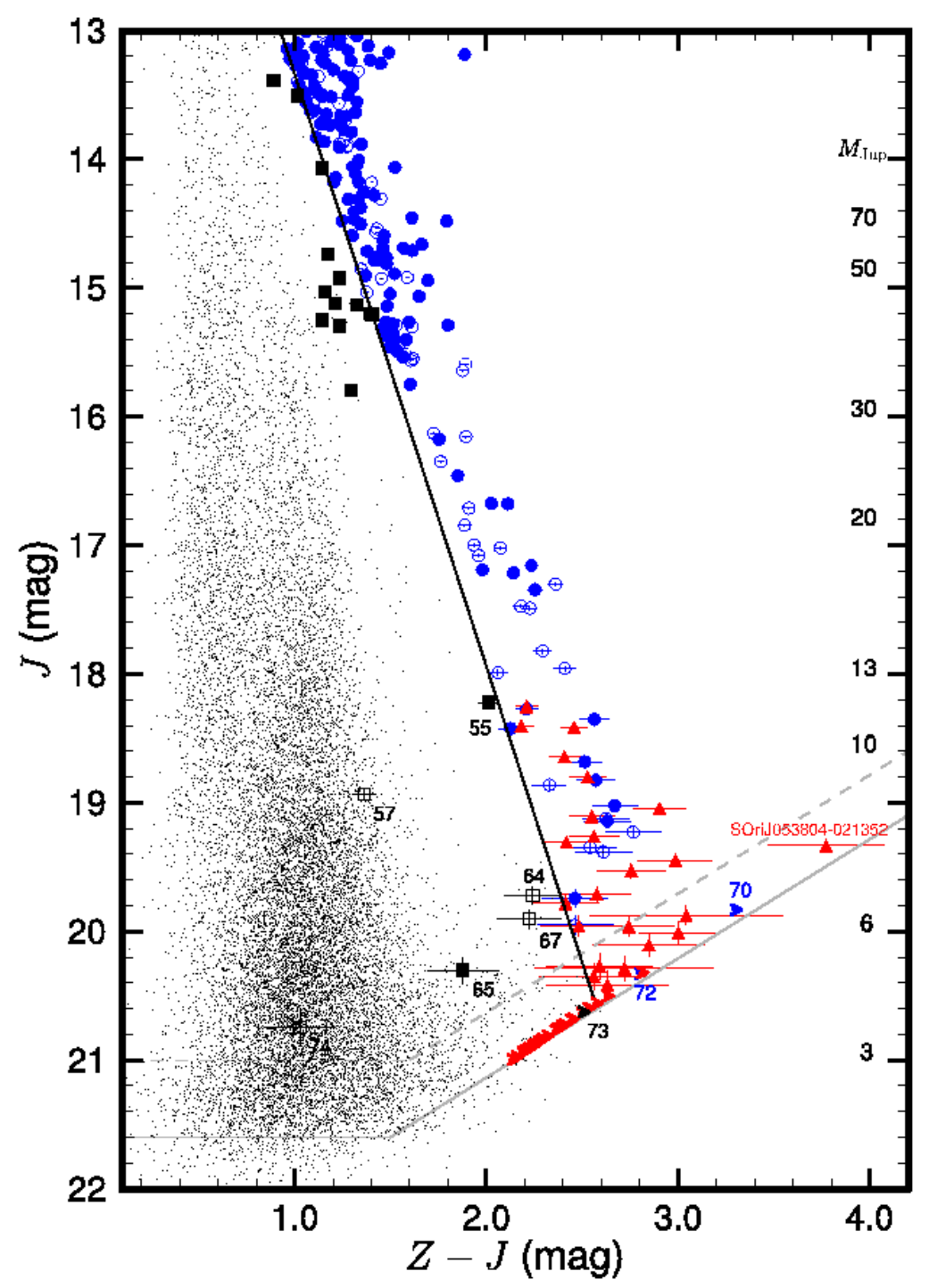

Fig. 2.-VISTA $J$ vs. $Z-J$ color magnitude diagram used to select $\sigma$ Orionis member candidates. Confirmed $\sigma$ Orionis members inside of the eligible zone are shown with filled blue circles, those outside of the eligible zone are in filled black squares. The straight line from $J=13$ to $20.5 \mathrm{mag}$ denotes the field-cluster separator according to our photometric criteria. Open blue circles stand for known cluster photometric candidates (not yet confirmed members) rediscovered with VISTA, those out of the eligible zone are represented with open black squares. The new discoveries $(J=18-$ $20.5 \mathrm{mag}$ ) are plotted as red triangles. S Ori sources discussed in this work and the new $\mathrm{T}$ type candidate are labeled. The sources of the extended survey (down to $J=21 \mathrm{mag}$ ) with no $Z$ detections are shown with red arrows. The completeness- and the $\sim 4-\sigma$ detection-limit are indicated with dashed and solid lines, respectively. Masses for an age of $3 \mathrm{Myr}$ and a distance of $352 \mathrm{pc}$ are labeled in Jupiter mass units. Field sources are plotted as black dots. A color version of this figure is available in the online journal. 

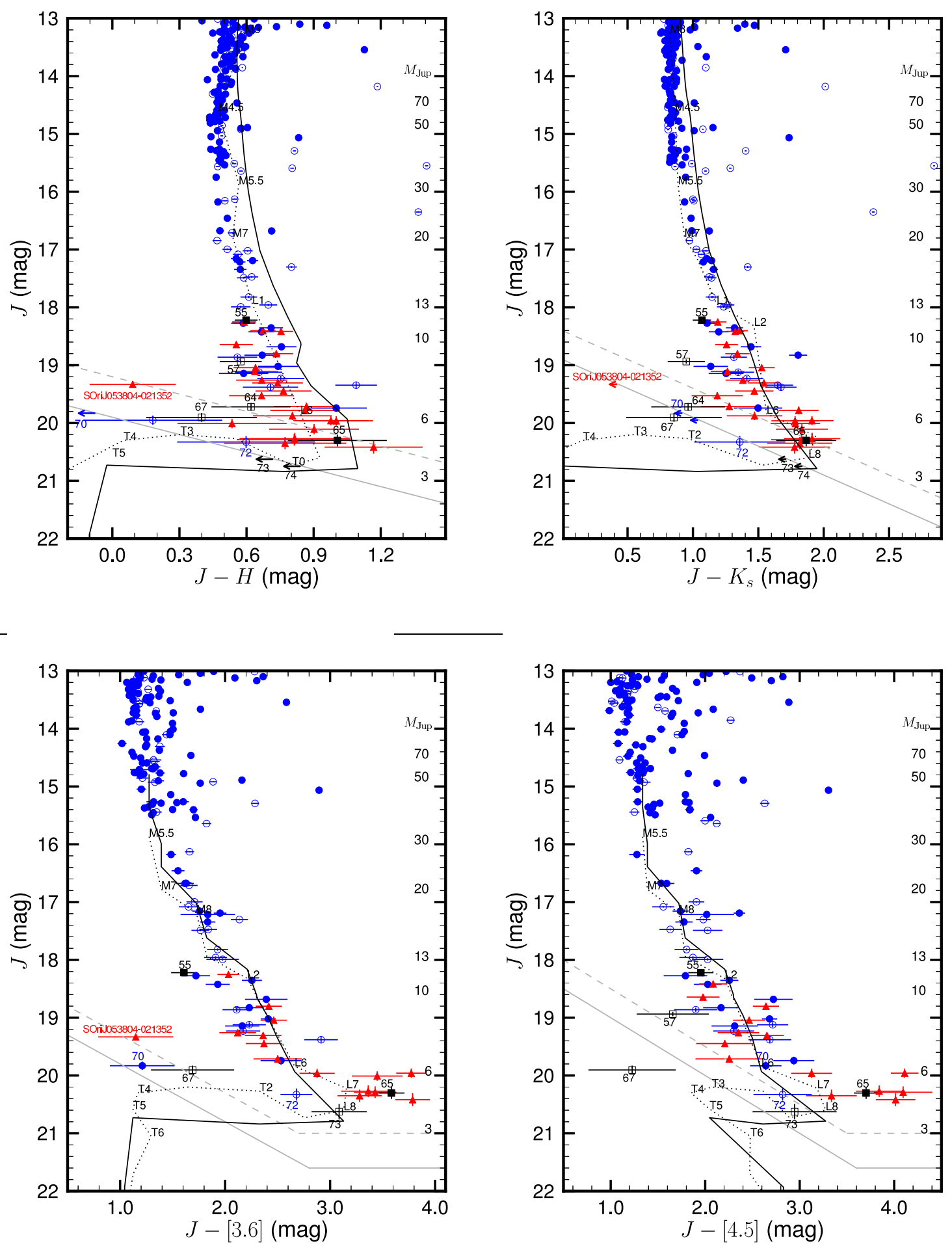

Fig. 3.- Various color-magnitude diagrams including VISTA and Spitzer data. Symbols are as in Figure 2. The mean sequence of M-L-T types for field objects is overlaid (dotted line), it is normalized at the $\sigma$ Orionis late-M types. The 3 Myr evolutionary model by Baraffe et al. (1998, 2003) and Chabrier et al. (2000) is included (solid line, see text). Source Mayrit 1082188 falls out the $J$ vs. $J-[4.5]$ diagram due to its red $J-[4.5]$ color. The color version of these figures is available in the online journal. 


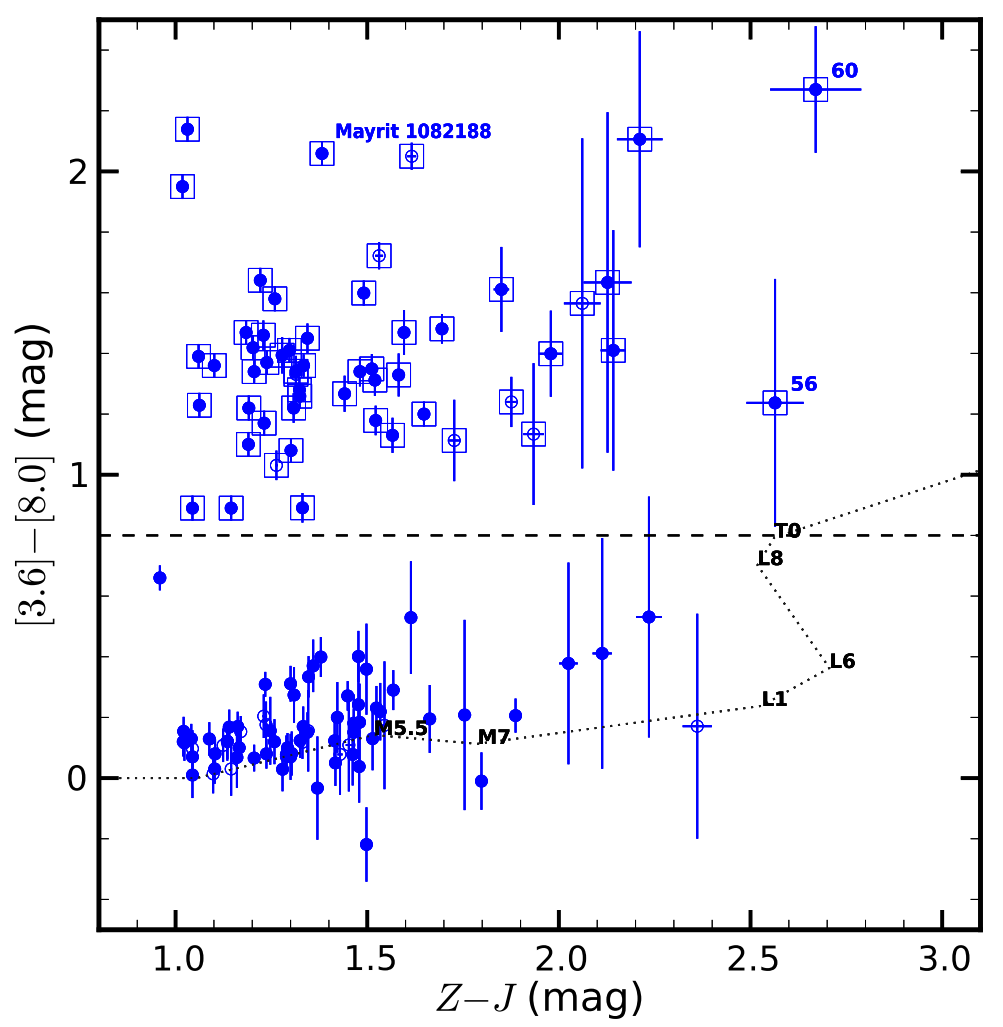

Fig. 4.- VISTA and IRAC/Spitzer [3.6] - [8.0] vs. $Z-J$ color-color diagram of all VISTA sources with data available in the four filters. Symbols are as in Figure 2. Objects with significant flux excesses at $8 \mu \mathrm{m}$ (framed circles) lie above the horizontal dashed line defined as the separator between objects with and without surrounding disks. The average location of early-M to T0 field dwarfs is indicated with the dotted line. Spectral types are labeled. Sources without infrared flux excesses nicely follow the field sequence. 


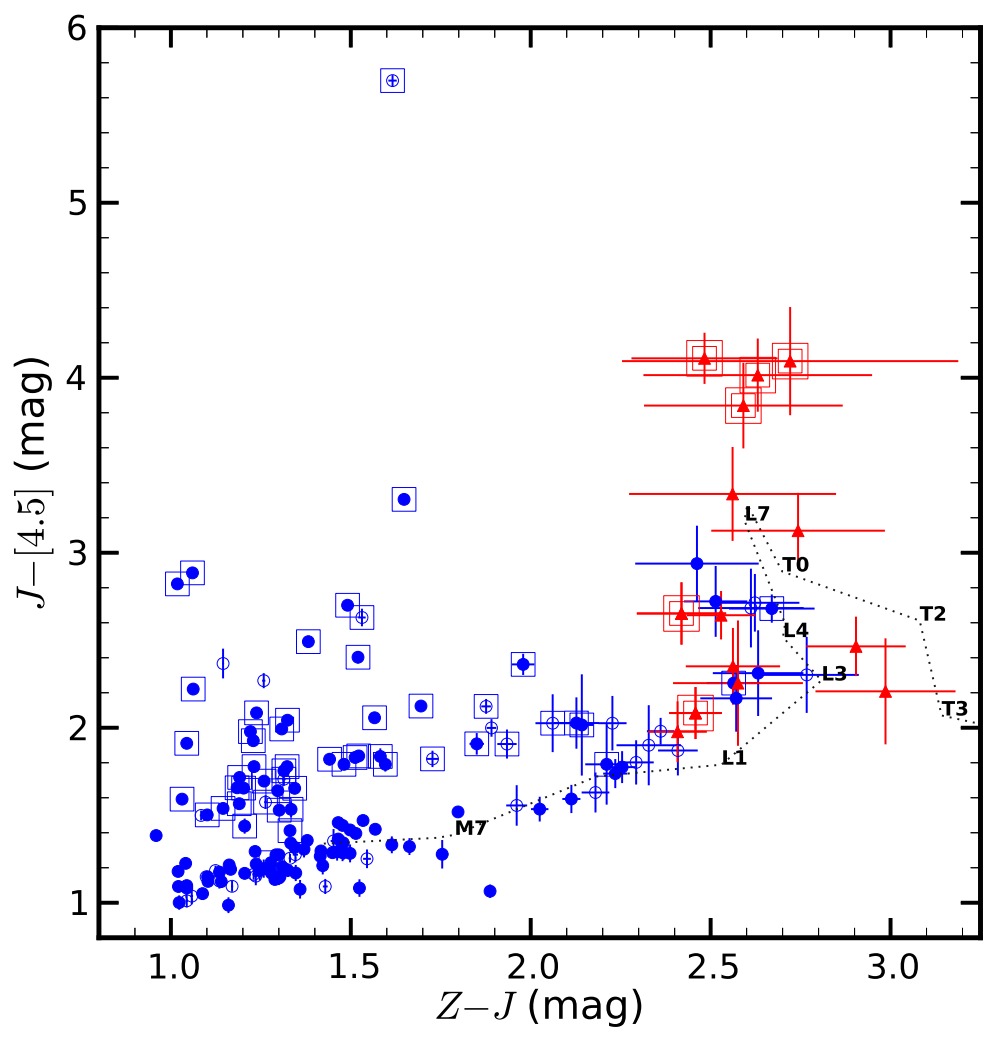

Fig. 5.- VISTA and IRAC/Spitzer $J-[4.5]$ vs. $Z-J$ color-color diagram of all VISTA sources with data available in the four filters. Symbols are as in Figure 2. Known $\sigma$ Orionis objects with flux excesses at $8 \mu \mathrm{m}$ have framed circles. The field sequence (early-M to early-T types) is indicated with a dotted line, and spectral types are labeled. New planetary mass candidates with $J-[4.5]$ colors indicative of flux excesses at $4.5 \mu \mathrm{m}$ are plotted as double framed red triangles. A color version of this figure is available in the online journal. 


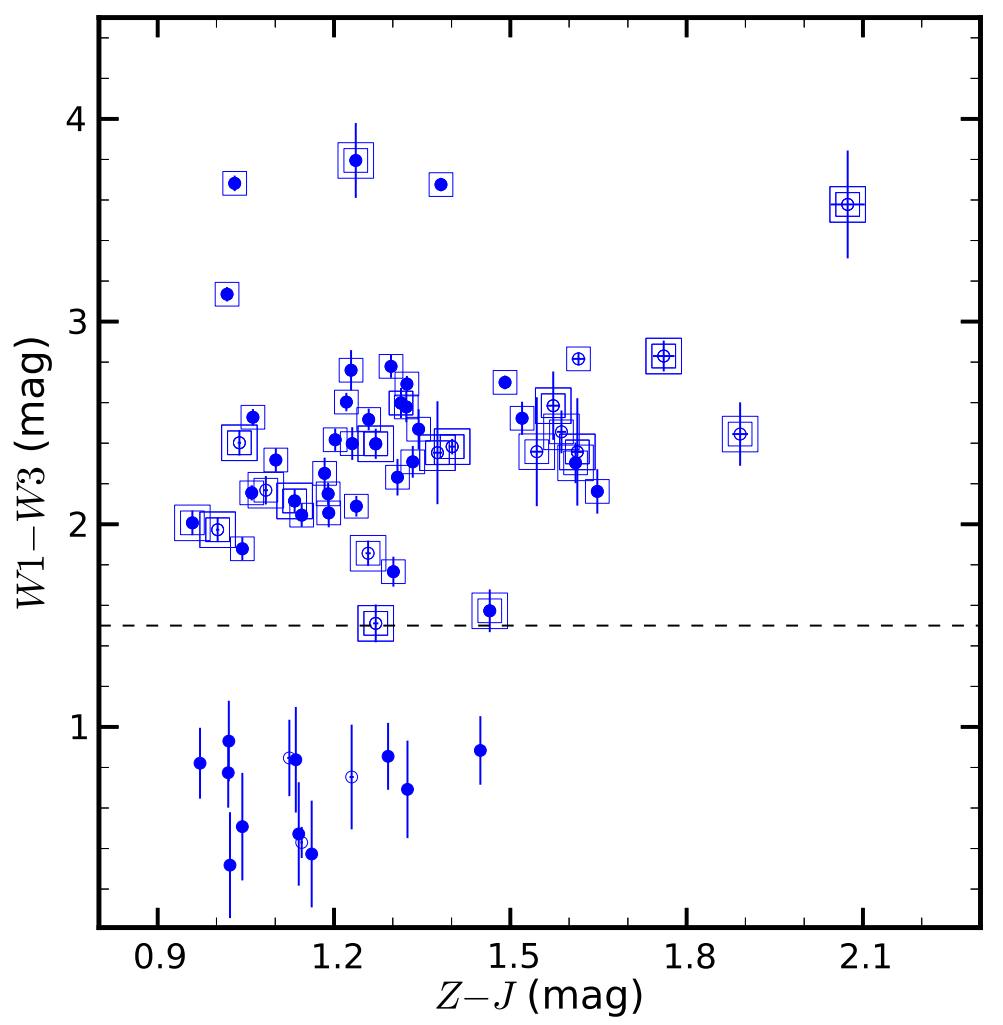

Fig. 6.- VISTA and WISE $Z-J$ vs. $W 1-W 3$ color-color diagram of all VISTA sources with data available in the four filters. Symbols are as in Figure 2. Sources with known IRAC/Spitzer flux excesses at $8 \mu \mathrm{m}$ are plotted as framed circles. All of them lie above the dashed horizontal line, which separates objects with and without flux excesses at $12 \mu \mathrm{m}$. The new WISE detections of $\sigma$ Orionis candidates that possibly harbor disks are shown as double framed cicles. 


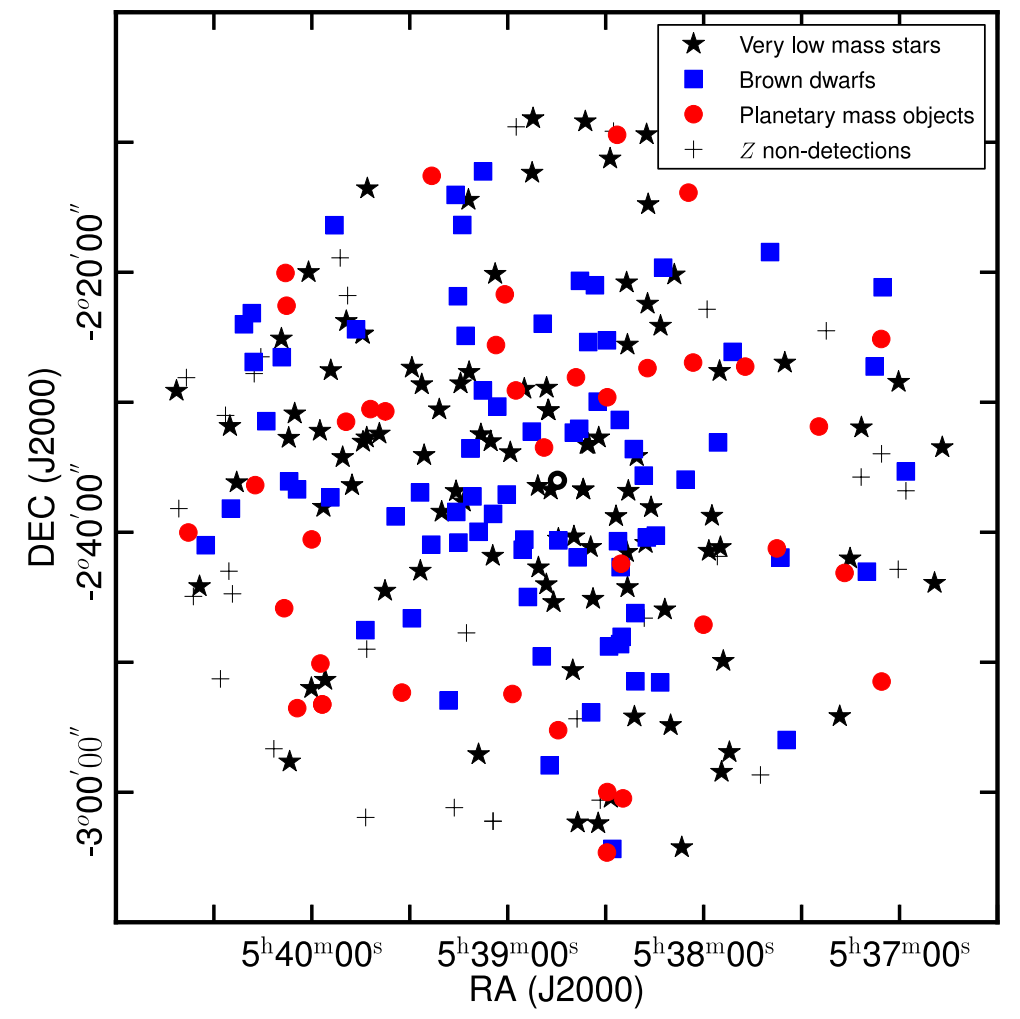

Fig. 7.- Spatial distribution of all $210 \sigma$ Orionis member candidates and $30 Z$-band non-detections found in the VISTA survey. The multiple, massive star $\sigma$ Ori is plotted as a central open circle. A color version of this figure is available in the online journal. 

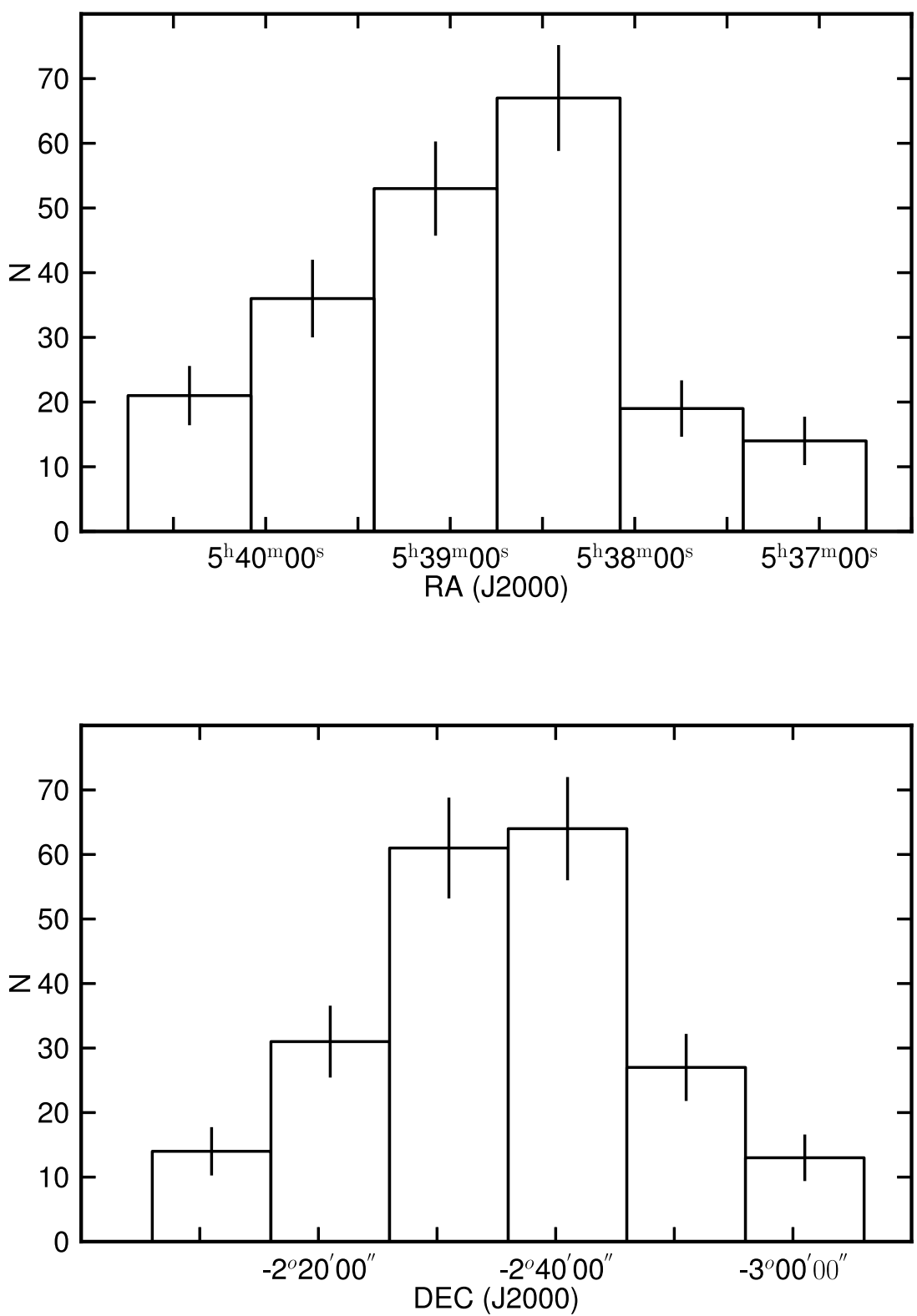

Fig. 8.- Distribution of the 210 VISTA sources in the right ascension (RA) and declination (DEC) coordinates. The size of the bins is $10^{\prime}$. All panels are centered at the location of the multiple, massive star $\sigma$ Ori (plotted as a cross symbol in Figure 7.) 


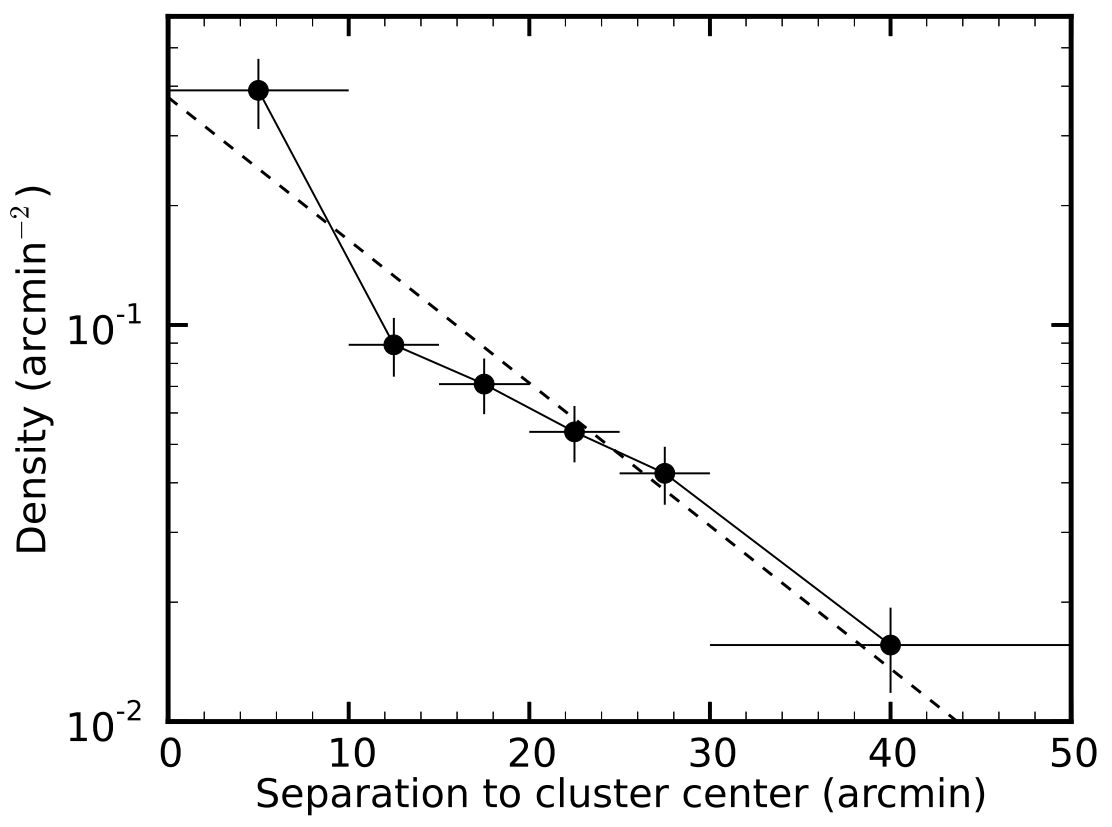

Fig. 9. - The radial surface density profile of all $210 \sigma$ Orionis member candidates is shown with dots and solid line. The profile extends up to $50^{\prime}$ from the cluster center. The exponential fit is depicted with a dashed line. 

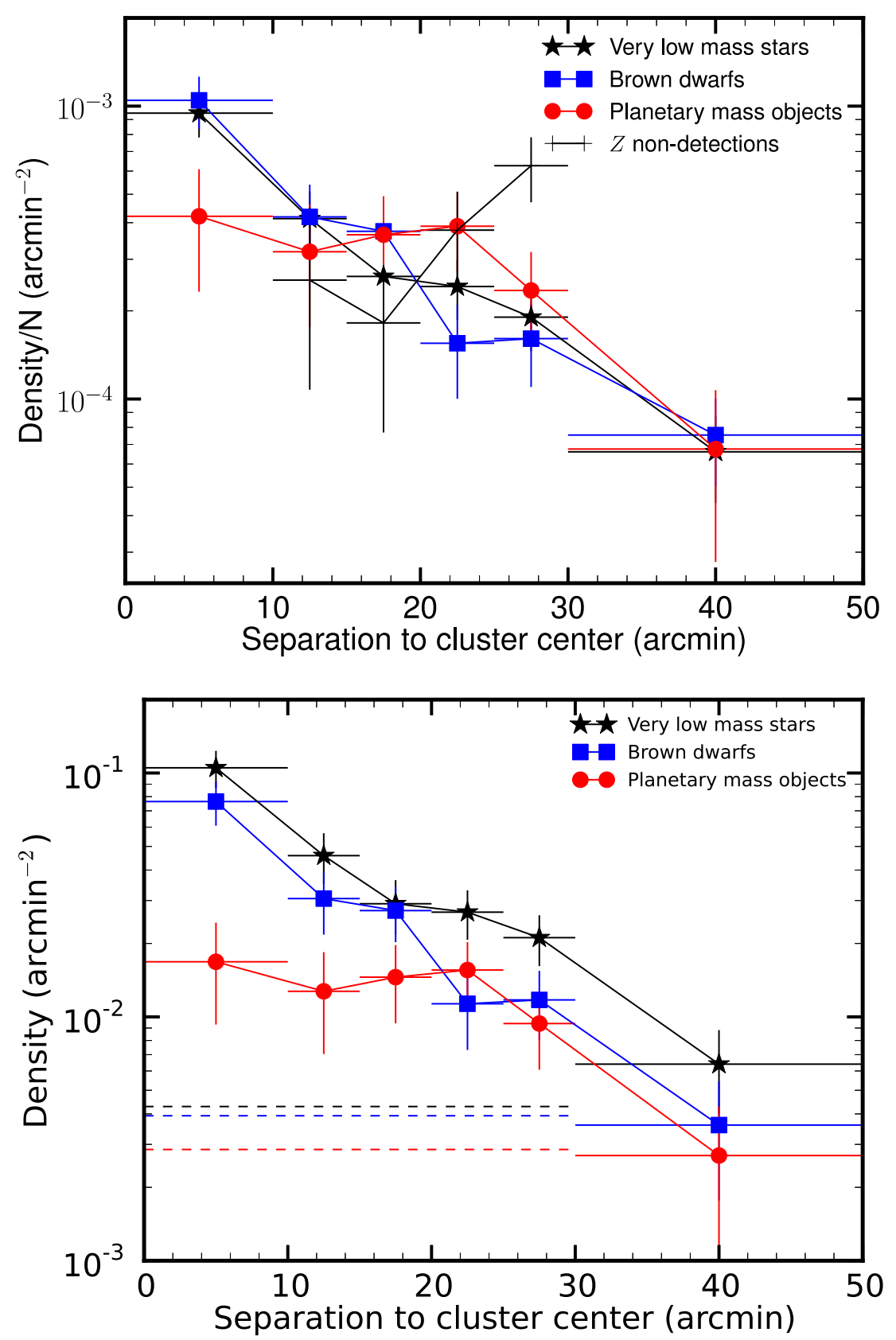

Fig. 10.- (Upper panel) Radial surface density profiles of the VISTA $\sigma$ Orionis candidates per mass (or magnitude) interval. The profiles extend up to a separation of $50^{\prime}$ from the cluster center. All profiles are normalized to the total number of objects in each mass interval. The surface densities have been measured for coronal regions of the following sizes: central circle of radius $=10^{\prime}$, from here up to $30^{\prime}$ the coronal step is $5^{\prime}$, and the outer region goes from $30^{\prime}$ to $50^{\prime}$ (see text). The vertical error bars correspond to Poisson uncertainties while the horizontal bars account for the size of their corresponding coronal regions. (Lower panel) Same as previous panel but displaying the surface densities as measured (without any normalization factor). The field dwarf contamination level for each mass (magnitude) interval is plotted as dashed lines (color codified). The color version of this figure is available in the online journal. 

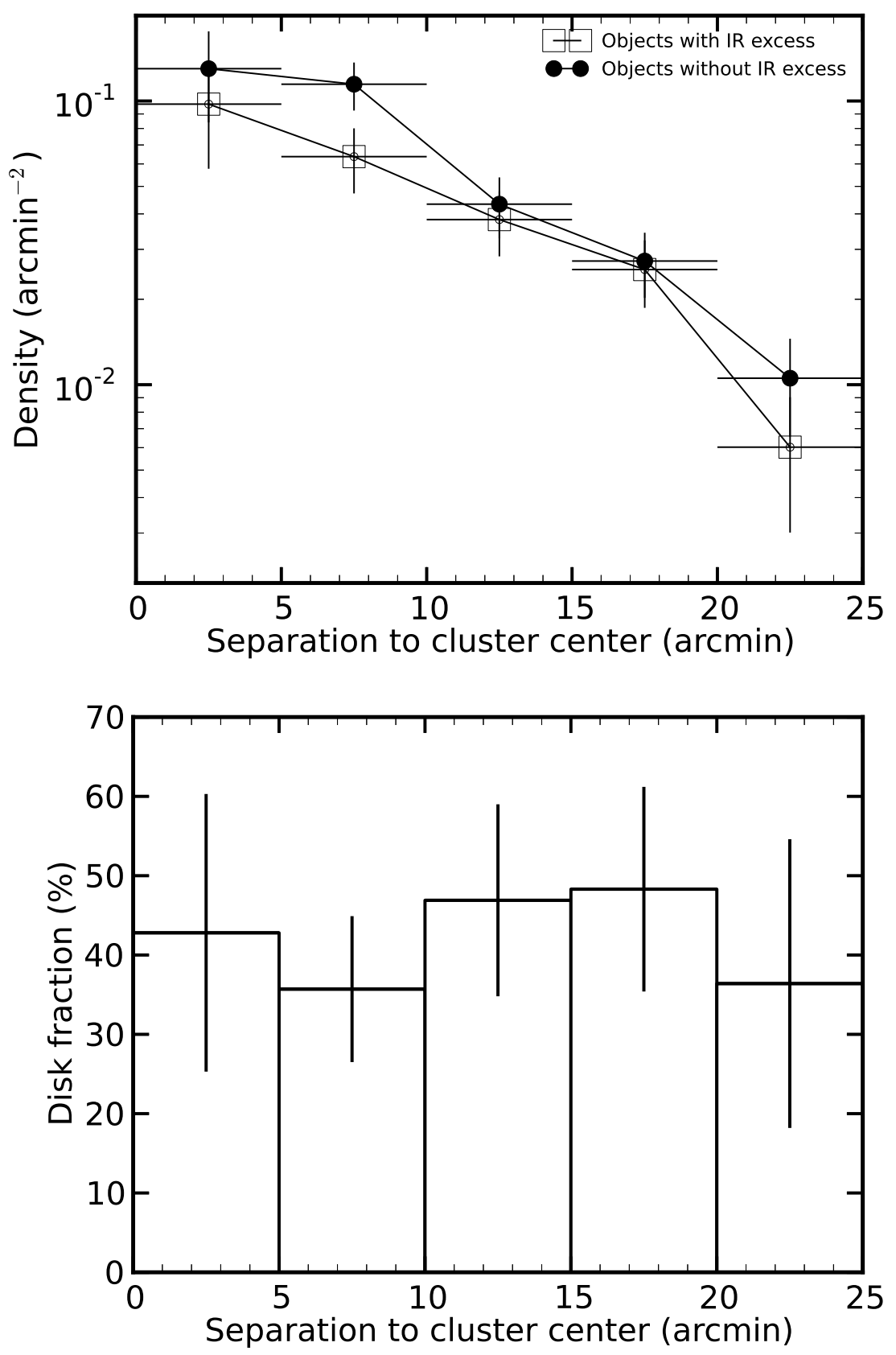

Fig. 11.- (Upper panel) Radial surface density profiles of $\sigma$ Orionis member candidates with (squares) and without (dots) infrared flux excesses at $8 \mu \mathrm{m}$. The coronal regions have a width of $5^{\prime}$ from the cluster center to a radial separation of $25^{\prime}$. Vertical error bars represent Poisson uncertainties, and horizontal bars account for the coronal size. (Lower panel) Disk fractions based on flux excesses at $8 \mu \mathrm{m}$ determined at different separations from the cluster center. 

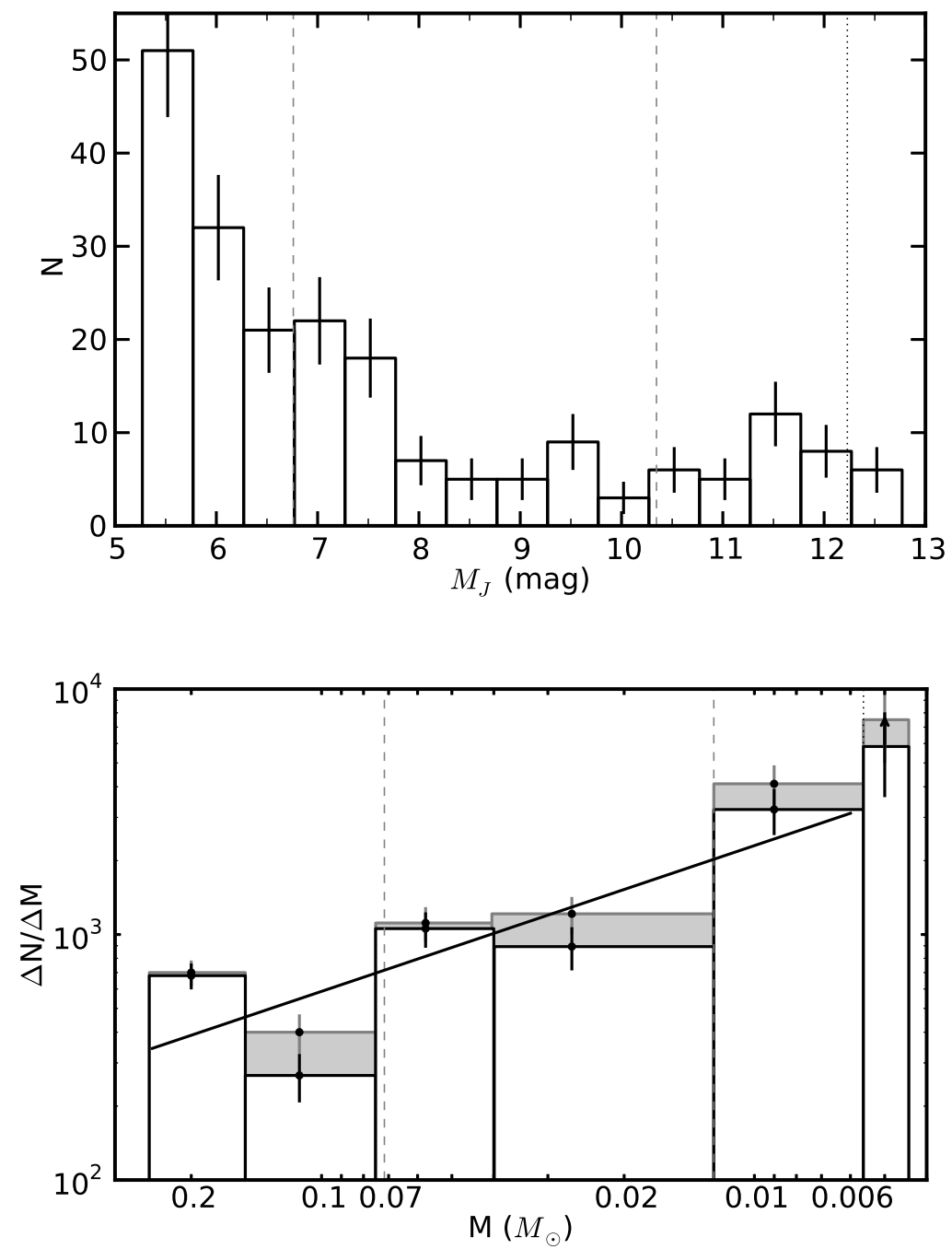

Fig. 12.- (Upper panel) The $J$-band luminosity function obtained from the 210 VISTA $\sigma$ Orionis candidates. From left to right, the vertical dashed lines and the dotted line denote the location of the substellar limit, the brown dwarf-planet borderline, and the completeness limit of the VISTA $Z J$ survey. Distance modulus is $\sim 7.73 \mathrm{mag}$ for a cluster distance of $352 \mathrm{pc}$. (Lower panel) The $\sigma$ Orionis low-mass spectrum $(\Delta N / \Delta M)$ derived for the mass interval $0.25-0.004 M_{\odot}$. The mass spectrum free of field contaminants is plotted as the open histogram, while the mass spectrum as measured is indicated by the grey upward extension of the mass bins. Vertical error bars stand for the Poisson count statistics. Vertical lines as in the upper panel of this Figure. The best power-law fit to the decontaminated mass spectrum is shown with a continuos line $\left(\Delta N / \Delta M \sim M^{-0.6 \pm 0.2}\right.$, obtained for the complete mass interval $0.25-0.006 M_{\odot}$ ). 


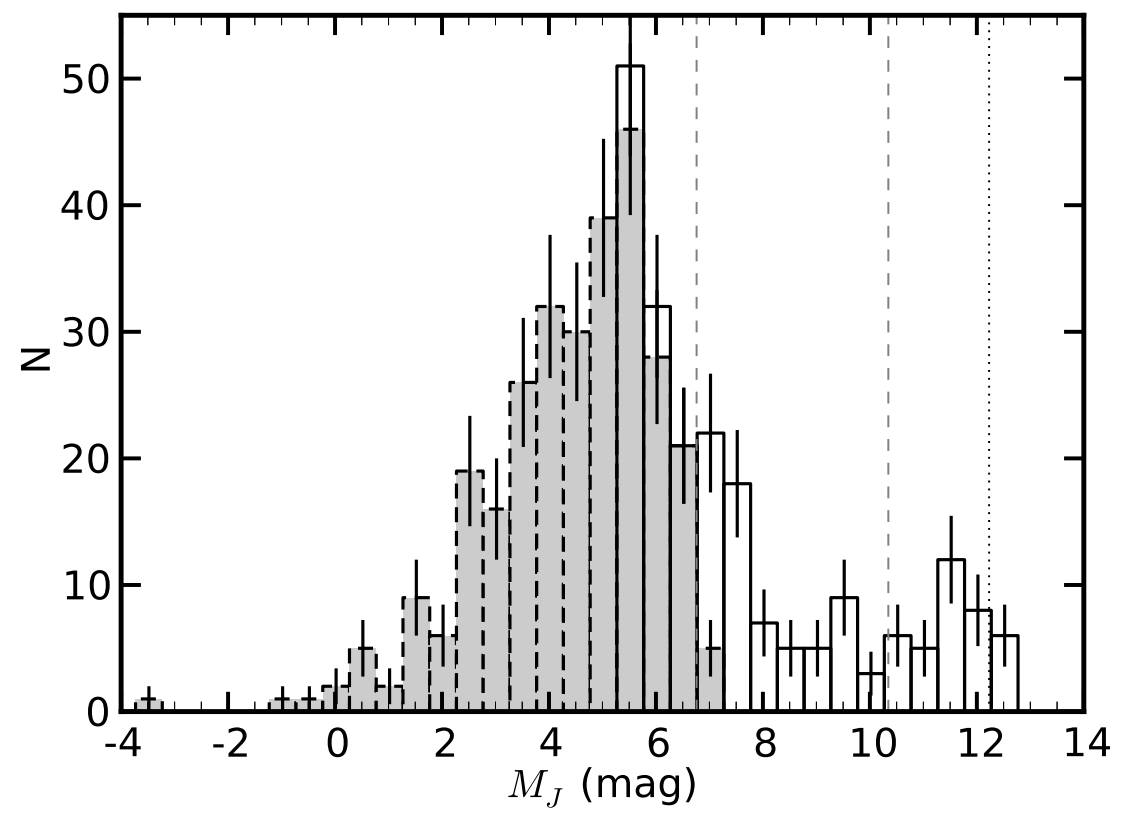

Fig. 13. - Combined Mayrit (grey dashed histogram) and VISTA (open histogram) $\sigma$ Orionis $J$ band luminosity function. From left to right, vertical lines as in Figure 12. Vertical error bars stand for the Poisson count statistics. The faintest bin of the Mayrit catalog remains largely incomplete. 


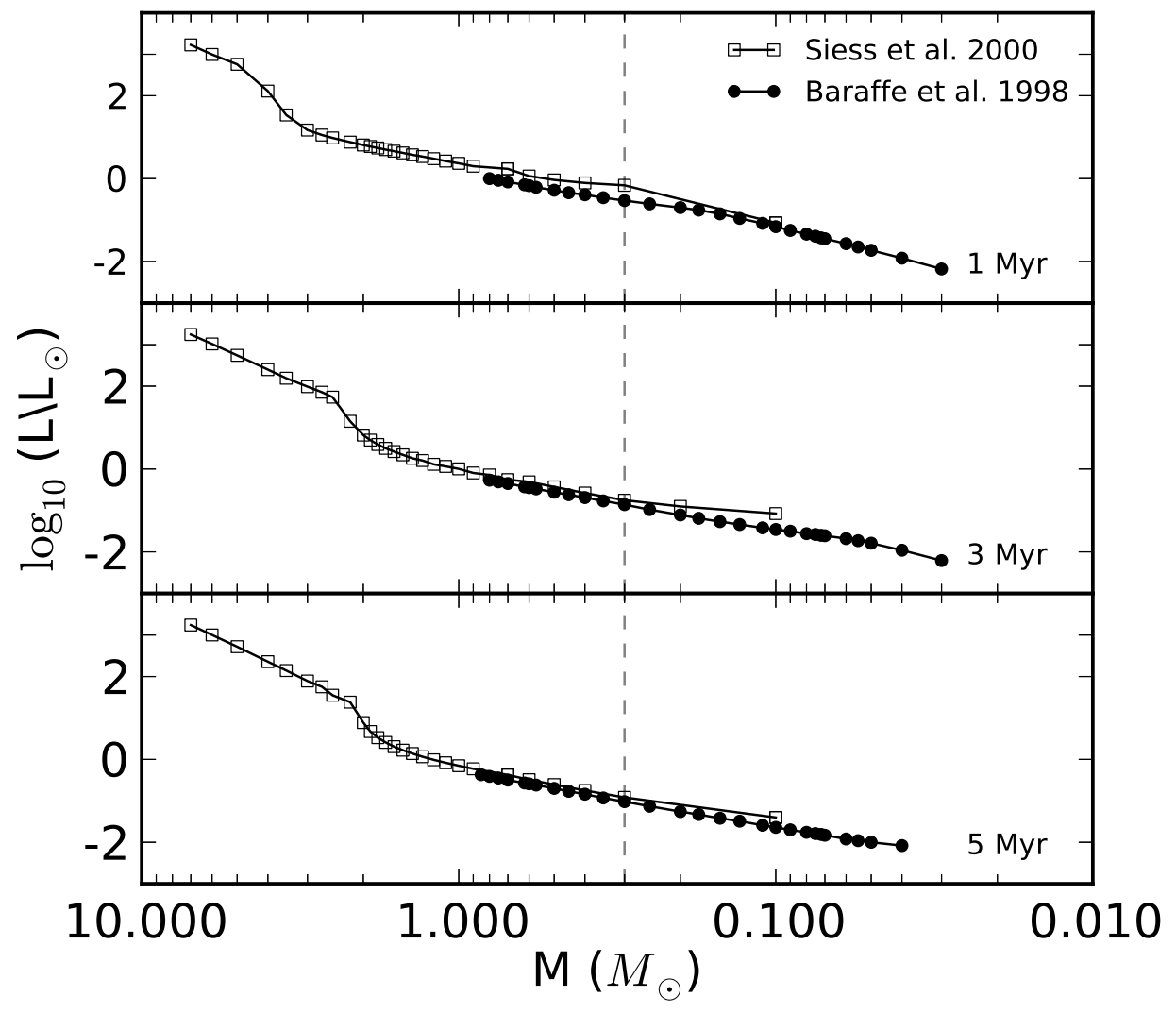

Fig. 14.- Theoretical mass-luminosity relations for 1, 3, and $5 \mathrm{Myr}$ given by Siess et al. (2000) and Baraffe et al. (1998). Solar metallicity is adopted. The vertical dashed line lies at $0.3 M_{\odot}$. 

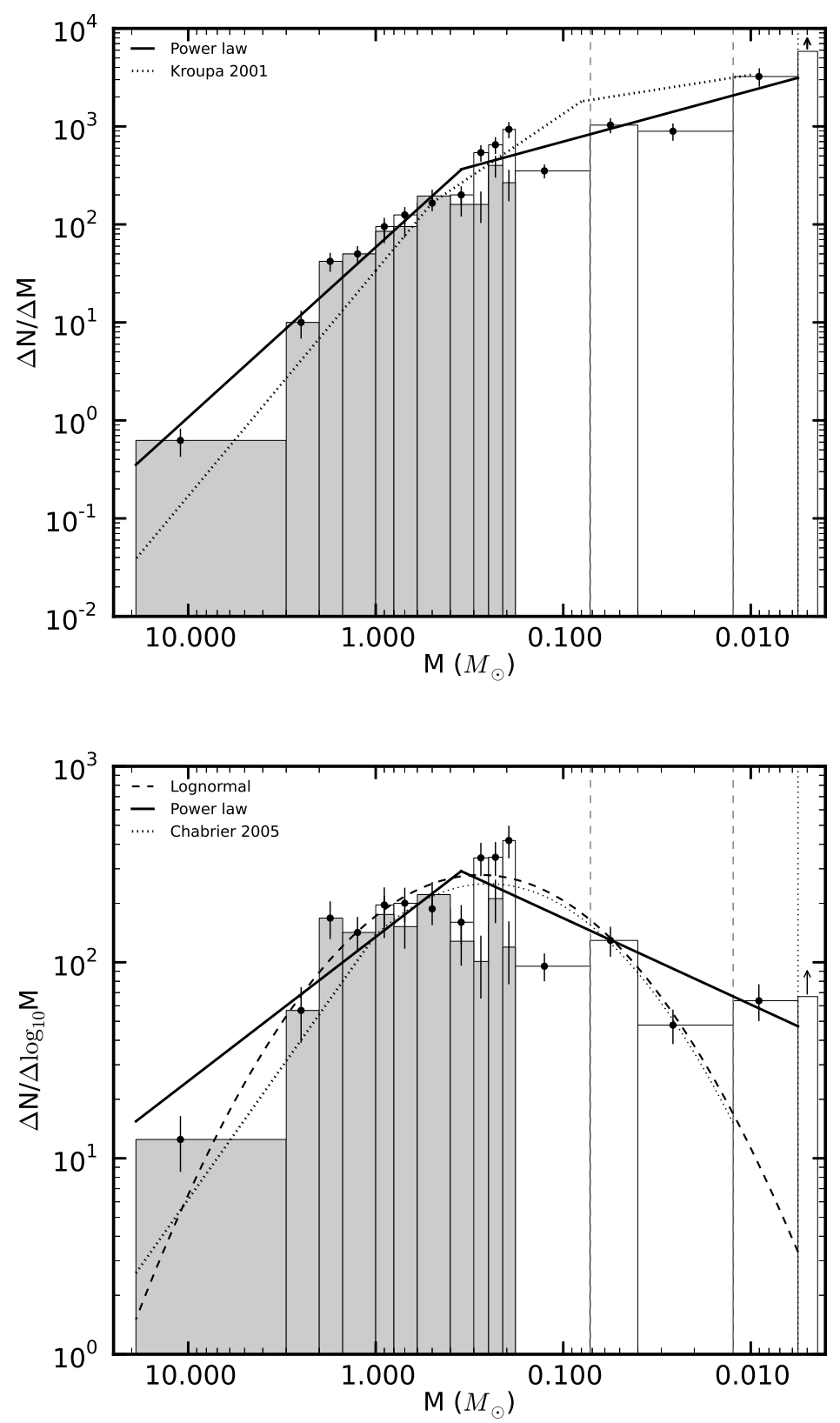

Fig. 15.- (Upper panel) $\sigma$ Orionis mass spectrum from 19 through $0.004 M_{\odot}$ using the Siess et al. (2000) models (grey area) and the Lyon models (open histogram, Baraffe et al. 1998; Chabrier et al. 2000; Baraffe et al. 2003). The best power-law fits to the mass domains $M>0.35 M_{\odot}$ and $M<0.35 M_{\odot}$ (using the Lyon models) are shown with continous lines. For comparison purposes, the mass spectrum derived by Kroupa (2001) is overlaid normalized to the total number of objects. (Lower panel) The $\sigma$ Orionis mass function using the Siess et al. (2000) models (grey area) and the Lyon models (open histogram). The best log-normal fit to our data is depicted with a dashed line, the double power-law fit from our mass spectrum is illustrated with a continuos line, and the Chabrier (2005) mass function normalized to the total number of sources is plotted as a dotted line. In both panels, from left to right, vertical lines as in Figure 12. Vertical error bars stand for the Poisson uncertainties. 
Table 1. Log of VISTA observational data on the $\sigma$ Orionis cluster

\begin{tabular}{|c|c|c|c|c|c|c|c|}
\hline $\begin{array}{l}\text { RA } \\
\left(\begin{array}{lll} & (J 2000 \\
h & m & s\end{array}\right)\end{array}$ & 0) $\left.{ }_{(\mathrm{O}}^{\mathrm{DEC}},{ }^{\prime \prime}\right)$ & Filter & $\begin{array}{c}\text { Observing date } \\
\text { (UT) }\end{array}$ & $\begin{array}{l}t_{\exp }^{a} \\
(\mathrm{~s})\end{array}$ & $\begin{array}{l}\text { Seeing } \\
(\operatorname{arcsec})\end{array}$ & $\begin{array}{l}\text { Completeness } \\
\text { (mag) }\end{array}$ & $\begin{array}{c}\text { 4- } \sigma \text { limit } \\
\text { (mag) }\end{array}$ \\
\hline \multirow[t]{5}{*}{053820.6} & -024746.0 & $Z$ & 2009 October 21,22 & 6084 & 0.8 & 22.6 & 23.1 \\
\hline & & $Y$ & 2009 October 21 & 1008 & 0.9 & 21.0 & 21.6 \\
\hline & & $J$ & 2009 October $20,21,24,28$ & 2112 & 0.9 & 21.0 & 21.6 \\
\hline & & $H$ & 2009 October 21 & 288 & 0.9 & 19.2 & 19.9 \\
\hline & & $K_{s}$ & 2009 October 21 & 288 & 0.7 & 18.4 & 18.9 \\
\hline
\end{tabular}

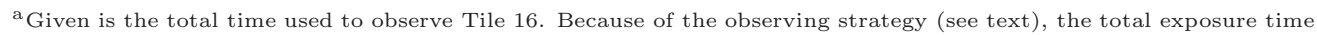
per pixel is typically a factor of one third of the exposure time listed.

Table 2. Central coordinates of the Suprime-Cam CCDs

\begin{tabular}{|c|c|c|}
\hline \multirow[t]{2}{*}{ CCD } & \multicolumn{2}{|c|}{ RA (J2000) DEC } \\
\hline & $\left(\begin{array}{lll}h & \mathrm{~m} & \mathrm{~s}\end{array}\right)$ & \\
\hline 0 & 053942.2 & -021108.0 \\
\hline 1 & 053914.1 & -021106.5 \\
\hline 2 & 053845.4 & -022456.0 \\
\hline 3 & 053816.9 & -022457.0 \\
\hline 4 & 053816.8 & -021106.0 \\
\hline 5 & 053844.4 & $\begin{array}{lll}-02 & 11 & 05.7\end{array}$ \\
\hline 7 & 053912.9 & -022456.6 \\
\hline 8 & 053747.7 & -022454.0 \\
\hline 9 & 053748.8 & -02 1109.0 \\
\hline
\end{tabular}


Table 3. Properties of $\sigma$ Orionis candidate members with youth features

\begin{tabular}{|c|c|c|c|c|c|c|c|}
\hline$\left(\begin{array}{lll} & \alpha & (\mathrm{J} 20 \\
\mathrm{h} & \mathrm{m} & \mathrm{s}\end{array}\right)$ & & $\begin{array}{ll}\delta & \\
, & \prime \prime\end{array}$ & Youth features $^{\mathrm{a}}$ & Envelope $^{\mathrm{b}}$ & $\begin{array}{l}\text { Infrared excess }{ }^{\mathrm{c}} \\
\qquad(\mu \mathrm{m})\end{array}$ & Alt. names & References \\
\hline 053836.88 & -02 & 3643. & $\mathrm{RV}, \mathrm{g}$ & $\checkmark$ & $\mathrm{N}$ & [SWW2004]J053836.759-023643.68,[MJO2008]J053836.8-023643 & 22,35 \\
\hline 053943.18 & -02 & 3243. & $\mathrm{~d}$ & $\checkmark$ & $\mathrm{Y}(4.5,8.0,12.0)$ & S Ori J053943.2-023243,[HHM2007]1182 & $20,22,31$ \\
\hline 053821.20 & -02 & 5411. & $\mathrm{~d}$ & $\checkmark$ & $\mathrm{Y}(4.5,8.0,12.0)$ & [SWW2004]J053821.194-025411.03,[HHM2007]467 & 22,31 \\
\hline 053850.78 & -02 & 3626 . & $\mathrm{Li}, \mathrm{g}, \mathrm{RV}, \mathrm{d}$ & $\checkmark$ & $\mathrm{Y}(12.0)$ & {$[$ KJN2005]8,[OJV2006]22 } & 24,28 \\
\hline 054009.32 & -02 & 2506. & $\mathrm{Li}, \mathrm{g}, \mathrm{RV}, \mathrm{d}$ & $\checkmark$ & $\mathrm{Y}(4.5,8.0,12.0)$ & S Ori J054009.3-022507,[KJN2005]10,[HHM2007]1362 & $20,24,31$ \\
\hline 053817.78 & -02 & 4050. & $\mathrm{~d}$ & $\checkmark$ & $\mathrm{Y}(4.5,8.0,12.0)$ & S Ori J053817.8-024050,[HHM2007]435 & $20,22,31$ \\
\hline 053929.35 & -02 & 2721. & $\mathrm{H} \alpha, \mathrm{d}$ & $\checkmark$ & $\mathrm{Y}(4.5,8.0,12.0)$ & Kiso A-0976 351 B,[HHM2007]1075 & 2,31 \\
\hline 054001.01 & -02 & 1959. & $\mathrm{Li}, \mathrm{g}, \mathrm{RV}, \mathrm{d}$ & $\checkmark$ & $\mathrm{N}$ & [SE2004]122,[KJN2005]6,[HHM2007]1323 & $19,24,31$ \\
\hline 053820.50 & -02 & 3408. & $\mathrm{Li}, \mathrm{H} \alpha, \mathrm{d}$ & $\checkmark$ & $\mathrm{Y}(4.5,8.0,12.0)$ & [W96]r053820-0234,[OJV2006]17 & $3,12,28$ \\
\hline 053828.65 & -02 & 1115. & $\mathrm{RV}, \mathrm{g}$ & $\checkmark$ & OL & [SE2004]20,[MJO2008]J053828.6-021115 & 19,35 \\
\hline 053926.77 & -02 & 4258. & $\mathrm{~d}$ & $\checkmark$ & $\mathrm{Y}(4.5,12.0)$ & [SWW2004]J053926.768-024258.25,[HHM2007]1057 & 22,31 \\
\hline 054023.01 & -02 & 3610. & $\mathrm{RV}, \mathrm{g}$ & $\checkmark$ & OL & [MJO2008]J054023.0-023610,SO430130 & 26,35 \\
\hline 053832.13 & -02 & 3243. & $\mathrm{~d}$ & $\checkmark$ & $\mathrm{Y}(8.0,12.0)$ & [FPS2006]NX 46,[HHM2007]566 & 27,31 \\
\hline 053834.60 & -02 & 4108. & $\mathrm{~d}, \mathrm{RV}, \mathrm{Li}, \mathrm{H} \alpha$ & $\checkmark$ & $\mathrm{Y}(4.5,8.0,12.0)$ & S Ori J053834.5-024109,[HHM2007]598,[SFR2008]S72 & $20,22,31,37$ \\
\hline 053859.22 & -02 & 3351. & $\mathrm{H} \alpha, \mathrm{d}, \mathrm{VR}, \mathrm{Li}$ & $\checkmark$ & $\mathrm{Y}(4.5,8.0,12.0)$ & Haro 5-18,[HHM2007]827,[SFR2008]S74 & $1,31,3$ ת \\
\hline 053846.85 & -02 & 3643. & $\mathrm{RV}, \mathrm{g}, \mathrm{d}, \mathrm{Li}$ & $\checkmark$ & $\mathrm{N}$ & [BNL2005]3.01 67,[HHM2007]721,[SFR2008]S83 & $25,31,35,37$ \\
\hline 053817.47 & -02 & 0923. & $\mathrm{RV}, \mathrm{Li}, \mathrm{g}$ & $\checkmark$ & OL & {$[\mathrm{SE} 2004] 122,[\mathrm{KJN} 2005] 3$} & 19,24 \\
\hline 053915.83 & -02 & 3650 . & $\mathrm{d}, \mathrm{RV}, \mathrm{Li}, \mathrm{H} \alpha$ & $\checkmark$ & $\mathrm{Y}(4.5,8.0,12.0)$ & [FPS2006]NX 151,[HHM2007]967,[SFR2008]S76 & $27,31,37$ \\
\hline 053808.98 & -02 & 2011. & $\mathrm{RV}, \mathrm{g}$ & $\checkmark$ & $\mathrm{N}$ & [SE2004]56,[MJO2008]J053808.9-022010 & 19,35 \\
\hline 053908.94 & -02 & 5704 & $\mathrm{Li}$ & $\checkmark$ & OL & [SWW2004]J053908.936-025704.91,SO411321 & 22,26 \\
\hline 053754.52 & -02 & 5826. & $\mathrm{RV}, \mathrm{Li}, \mathrm{g}$ & $\checkmark$ & OL & {$[\mathrm{KJN} 2005] 23$} & 24 \\
\hline 053957.53 & -02 & 3212. & $\mathrm{RV}, \mathrm{g}$ & $\checkmark$ & $\mathrm{N}$ & S Ori J053957.5-023212,[MJO2008]J053957.5-023212 & 20,35 \\
\hline 053758.40 & -02 & 4126. & $\mathrm{RV}, \mathrm{Li}, \mathrm{g}$ & $\checkmark$ & $\mathrm{N}$ & [SWW2004]J053758.401-024126.16,[KJN2005]18 & $22,24,35$ \\
\hline 053823.54 & -02 & 4131. & $\mathrm{~d}, \mathrm{~g}, \mathrm{RV}$ & $\checkmark$ & $\mathrm{N}$ & S Ori J053823.6-024132,[BNL2005]229 & $20,22,25,35$ \\
\hline 053920.97 & -02 & 3033. & $R V, L i, g, d$ & $\checkmark$ & $\mathrm{N}$ & S Ori 3,[KJN2005]20,[OJV2006]24 & $5,24,28,31$ \\
\hline 053914.46 & -02 & 2833. & $\mathrm{H} \alpha, \mathrm{d}, \mathrm{RV}$ & $\checkmark$ & $\mathrm{N}$ & S Ori J053914.5-022834,[OJV2004]25,[SFR2008]S75 & $12,28,31,35$ \\
\hline 053956.45 & -02 & 3803. & $\mathrm{RV}, \mathrm{d}, \mathrm{g}$ & $\checkmark$ & $\mathrm{Y}(4.5,8.0,12.0)$ & S Ori J053956.4-023804,[BNL2005]8.02 143,[HHM2007]1285,[MJO2008] J053956.4-023803 & $20,25,31$ \\
\hline 053844.49 & -02 & 4030. & $\mathrm{RV}, \mathrm{Li}, \mathrm{g}$ & $\checkmark$ & $\mathrm{N}$ & S Ori J053844.4-024030,[KJN2005]5 & 20,24 \\
\hline 053905.24 & -02 & 3300. & $\mathrm{RV}, \mathrm{Li}, \mathrm{g}$ & $\checkmark$ & $\mathrm{N}$ & [SWW2004]J053905.247-023300.52,[KJN2005]4 & 22,24 \\
\hline 054007.08 & -02 & 3244 . & $\mathrm{RV}, \mathrm{g}$ & $\checkmark$ & $\mathrm{N}$ & S Ori J054007.1-023245,[MJO2008]J054007.0-023244 & $20,31,35$ \\
\hline 053823.59 & -02 & 2047 . & $\mathrm{RV}, \mathrm{Li}, \mathrm{g}, \mathrm{d}$ & $\checkmark$ & $\mathrm{Y}(4.5,8.0,12.0)$ & [SE2004]15,[KJN2005]13,[HHM2007]490 & $19,24,31,35$ \\
\hline 053752.11 & -02 & 5655. & $\mathrm{RV}, \mathrm{Li}, \mathrm{g}$ & $\checkmark$ & OL & [SWW2004]J053752.099-025655.26,[KJN2005]14 & 22,24 \\
\hline 053926.47 & -02 & 2615. & $\mathrm{Li}$ & $\cdots$ & $\mathrm{N}$ & {$[\mathrm{SE2004}] 94, \mathrm{SO} 110611$} & 19,26 \\
\hline 053939.32 & -02 & 3225 . & $\mathrm{d}, \mathrm{RV}, \mathrm{g}$ & $\checkmark$ & $\mathrm{N}$ & S Ori 4,[OJV2006]29,[MJO2008]J053939.3-023225 & $5,28,31,35$ \\
\hline
\end{tabular}


Table 3-Continued

\begin{tabular}{|c|c|c|c|c|c|c|c|}
\hline$\left(\begin{array}{lll} & \alpha & (\mathrm{J} 20 \\
\mathrm{h} & \mathrm{m} & \mathrm{s}\end{array}\right)$ & & $\begin{array}{ll}\delta & \\
, & \prime \prime\end{array}$ & Youth features ${ }^{\mathrm{a}}$ & Envelope $^{\mathrm{b}}$ & $\begin{array}{l}\text { Infrared excess }{ }^{\mathrm{c}} \\
\qquad(\mu \mathrm{m})\end{array}$ & Alt. names & References \\
\hline 053847.66 & -02 & 3037. & $\mathrm{RV}, \mathrm{Li}, \mathrm{g}$ & $\checkmark$ & $\mathrm{N}$ & S Ori $6,[$ KJN2005]17 & 5,24 \\
\hline 053947.69 & -02 & 3623. & $\mathrm{RV}, \mathrm{g}$ & $\checkmark$ & $\mathrm{N}$ & [BNL2005]8.02 179,[MJO2008]J053947.6-023622 & 25,35 \\
\hline 053823.32 & -02 & 4414. & $\mathrm{RV}, \mathrm{Li}, \mathrm{g}$ & $\checkmark$ & $\mathrm{N}$ & S Ori J053823.3-024414,[KJN2005]15 & $20,24,35$ \\
\hline 053949.44 & -02 & 2345. & $\mathrm{RV}, \mathrm{Li}, \mathrm{H} \alpha, \mathrm{d}$ & $\checkmark$ & $\mathrm{Y}(4.5,8.0,12.0)$ & S Ori J053949.3-022346, [HHM2007]1230 & 12,31 \\
\hline 053954.20 & -02 & 2732. & $\mathrm{~d}$ & $\checkmark$ & $\mathrm{Y}(4.5,8.0,12.0)$ & S Ori J053954.2-022733,[HHM2007]1266 & 20,31 \\
\hline 053926.33 & -02 & 2837. & $\mathrm{~d}$ & $\checkmark$ & $\mathrm{Y}(4.5,8.0,12.0)$ & S Ori $2,[$ HHM2007]1050 & 5,31 \\
\hline 054025.09 & -02 & 3150. & $R V, g$ & $\checkmark$ & $\mathrm{OL}, \mathrm{Y}(12.0)$ & [MJO2008]J054025.1-023149 & 35 \\
\hline 053845.97 & -02 & 4523. & $R V, g$ & $\checkmark$ & $\mathrm{N}$ & S Ori J053845.9-024523,[MJO2008]J053845.9-024523 & 20,35 \\
\hline 053816.10 & -02 & 3804. & $\mathrm{RV}, \mathrm{Li}, \mathrm{g}$ & $\checkmark$ & $\mathrm{N}$ & S Ori J053816.0-023805,[KJN2005]11 & $20,24,35$ \\
\hline 053901.16 & -02 & 3638. & $\mathrm{RV}, \mathrm{Li}, \mathrm{g}$ & $\cdots$ & $\mathrm{N}$ & {$[\mathrm{KJN} 2005] 9$} & 24,35 \\
\hline 053646.91 & -02 & 3328. & $\mathrm{RV}, \mathrm{Li}, \mathrm{g}$ & $\checkmark$ & OL & [SWW2004]J053646.923-023328.20,[KJN2005]16 & 22,24 \\
\hline 053754.86 & -02 & 4109. & $\mathrm{RV}, \mathrm{g}, \mathrm{d}$ & $\checkmark$ & $\mathrm{Y}(4.5,8.0,12.0)$ & [SWW2004]J053754.858-024109.19,[BNL2005]4.03 237,[HHM2007]247 & $22,25,31$ \\
\hline 053904.59 & -02 & 4149. & $\mathrm{RV}, \mathrm{Li}, \mathrm{H} \alpha, \mathrm{d}$ & $\checkmark$ & $\mathrm{Y}(4.5,8.0,12.0)$ & [FPS2006]NX 130,[HHM2007]871,[SFR2008]S98 & $27,31,37$ \\
\hline 053817.18 & -02 & 2225. & $\mathrm{RV}, \mathrm{g}$ & $\checkmark$ & $\mathrm{N}$ & S Ori 9,[MJO2008]J053817.1-022225 & 5,35 \\
\hline 053715.15 & -02 & 4201. & $\mathrm{RV}, \mathrm{Li}, \mathrm{H} \alpha$ & $\checkmark$ & $\mathrm{N}$ & S Ori J053715.1-024202 & 12 \\
\hline 053950.56 & -02 & 3413. & $\mathrm{RV}, \mathrm{Li}, \mathrm{g}$ & $\checkmark$ & $\mathrm{N}$ & S Ori J053950.6-023414,[KJN2005]19 & $20,24,35$ \\
\hline 053852.24 & -02 & 0809. & $\mathrm{RV}, \mathrm{g}$ & $\checkmark$ & OL & [SWW2004]J053852.241-020809.73,[MJO2008]J053852.2-020809 & 22,35 \\
\hline 053840.08 & -02 & 5037. & $\mathrm{RV}, \mathrm{Li}, \mathrm{g}$ & $\checkmark$ & $\mathrm{N}$ & {$[$ SWW2004]J053840.078-025037.14, $[\mathrm{KJN} 2005] 22$} & $22,24,35$ \\
\hline 054034.39 & -02 & 4409. & $\mathrm{RV}, \mathrm{g}$ & $\checkmark$ & $\mathrm{OL}, \mathrm{Y}(12.0)$ & {$[\mathrm{BNL} 2005] 2.0363$} & 25,35 \\
\hline 053823.34 & -02 & 2534. & $\mathrm{~d}$ & $\checkmark$ & $\mathrm{Y}(4.5,8.0,12.0)$ & [SWW2004]J053823.351-022534.51,[HHM2007]485 & 22,31 \\
\hline 053839.72 & -02 & 4019. & $R V, g$ & $\checkmark$ & $\mathrm{N}$ & [HHM2007]655,[MJO2008]J053839.7-024019 & 31,35 \\
\hline 053854.93 & -02 & 2858. & $\mathrm{RV}, \mathrm{Li}, \mathrm{g}$ & $\checkmark$ & $\mathrm{N}$ & [SWW2004]J053854.916-022858.24,[KJN2005]21 & 22,24 \\
\hline 053823.07 & -02 & 3649. & $\mathrm{RV}, \mathrm{g}, \mathrm{d}$ & $\checkmark$ & $\mathrm{Y}(4.5,8.0,12.0)$ & [SWW2004]J053822.999-023649.48,[BNL2005]3.01 51,[HHM2007]482 & $22,25,31$ \\
\hline 053903.86 & -02 & 2008. & $\mathrm{~d}$ & $\checkmark$ & $\mathrm{Y}(4.5,8.0,12.0)$ & [SWW2004]J053903.866-022007.8,[HHM2007]866 & 22,31 \\
\hline 053908.22 & -02 & 3228. & d & $\checkmark$ & $\mathrm{N}$ & S Ori $7,[$ OJV2006] 38 & $5,28,31$ \\
\hline 053850.60 & -02 & 4242. & $\mathrm{RV}, \mathrm{Li}, \mathrm{g}, \mathrm{d}$ & $\checkmark$ & $\mathrm{Y}(4.5,8.0,12.0)$ & S Ori J053850.6-024244,[KJN2005]31,[HHM2007]762 & $20,24,31,35$ \\
\hline 053943.00 & -02 & 1333. & $\mathrm{RV}, \mathrm{Li}, \mathrm{g}$ & $\checkmark$ & OL & [SWW2004]J053942.989-021333.18,[KJN2005]24 & $22,24,35$ \\
\hline 053756.14 & -02 & 0926. & $\mathrm{RV}, \mathrm{g}, \mathrm{d}$ & $\checkmark$ & OL & [SWW2004]J053756.130-020926.54,[KJN2005]27,[OJV2006]41 & $22,24,28,35$ \\
\hline 053835.29 & -02 & 3313. & $\mathrm{RV}, \mathrm{Li}$ & $\checkmark$ & $\mathrm{N}$ & [HHM2007]606,[SFR2008]S88 & 31,37 \\
\hline 054005.25 & -02 & 3052. & $\mathrm{RV}, \mathrm{Li}, \mathrm{H} \alpha, \mathrm{d}$ & $\checkmark$ & $\mathrm{Y}(4.5,8.0,12.0)$ & S Ori J054005.1-023052,[HHM2007]1344 & $12,31,35$ \\
\hline 053848.18 & -02 & 4400. & $\mathrm{RV}, \mathrm{Li}, \mathrm{g}, \mathrm{d}$ & $\checkmark$ & $\mathrm{Y}(4.5,8.0,12.0)$ & S Ori J053848.1-024401,[KJN2005]35,[HHM2007]739 & $20,24,31,35$ \\
\hline 053826.84 & -02 & 3846. & $R V, g, d$ & $\checkmark$ & $\mathrm{Y}(4.5,8.0,12.0)$ & S Ori J053826.8-023846,[BNL2005]4.03 368,[HHM2007]514 & $20,25,31,35,37$ \\
\hline 053813.21 & -02 & 2407. & $\mathrm{H} \alpha, \mathrm{RV}, \mathrm{g}$ & $\checkmark$ & $\mathrm{N}$ & S Ori 13,[MJO2008]J053813.2-022407 & $5,15,35$ \\
\hline 054000.14 & -02 & 5159. & Li,g,RV & $\checkmark$ & $\mathrm{N}$ & S Ori J054000.2-025159,[KJN2005]46,[MJO2008] J054000.1-025159 & $21,24,35$ \\
\hline
\end{tabular}


Table 3-Continued

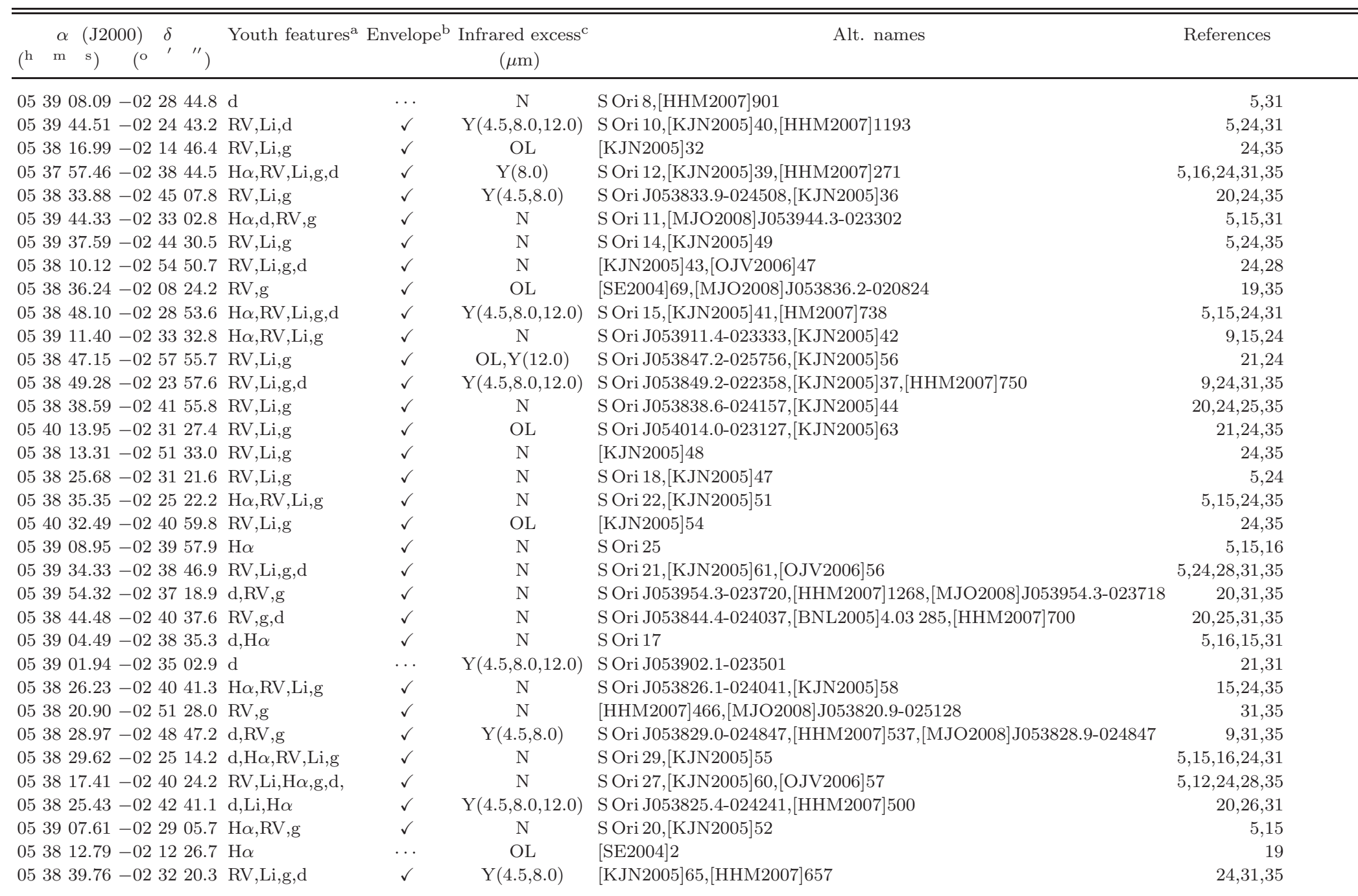


Table 3-Continued

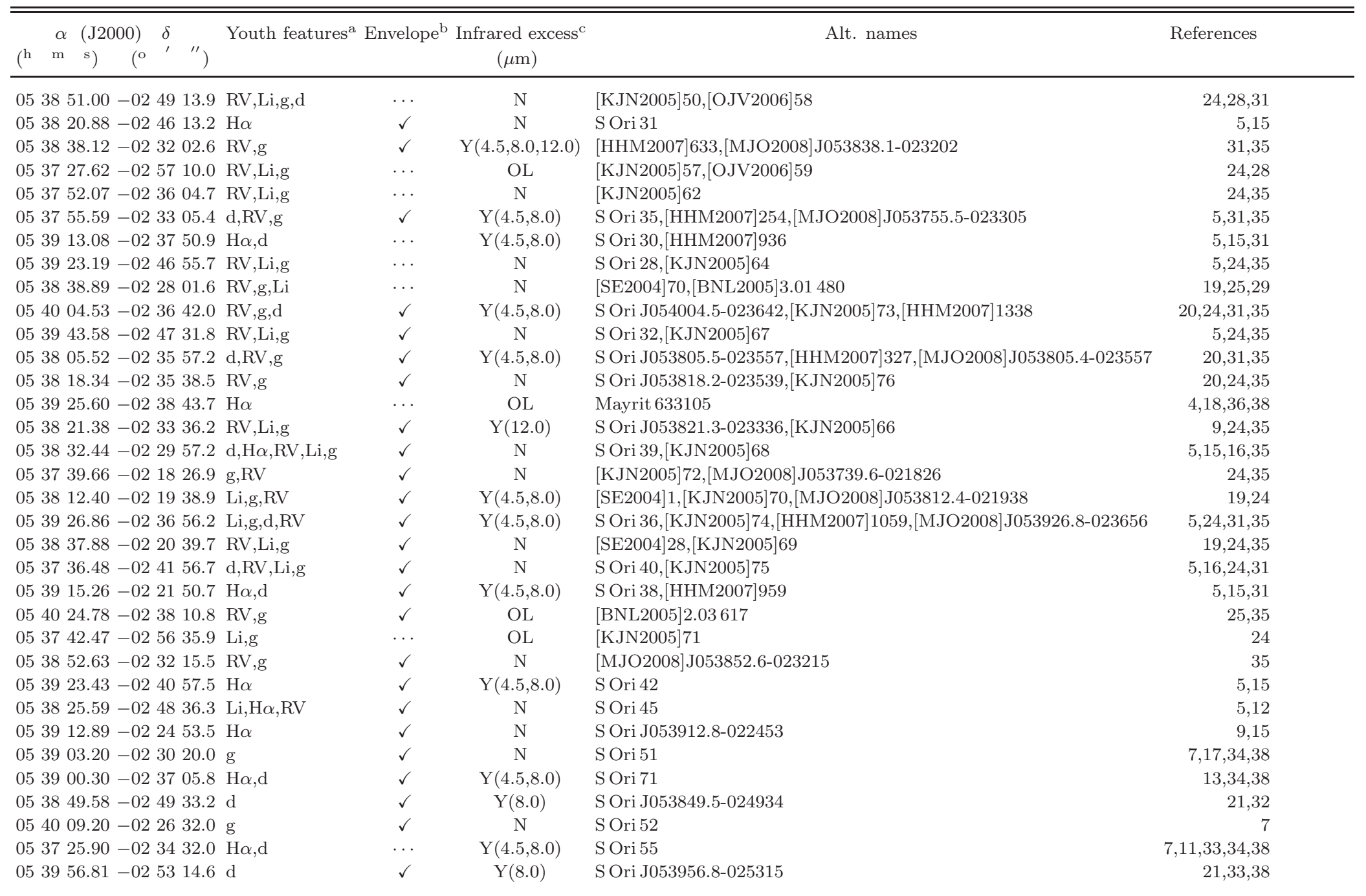


Table 3-Continued

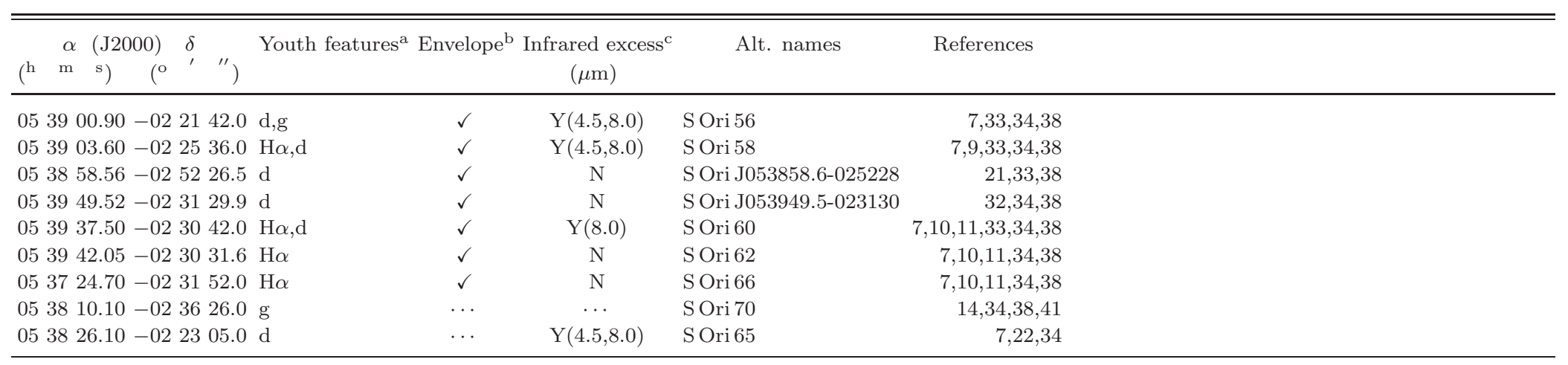

a "RV" stands for radial velocity consistent with the systemic cluster radial velocity; "Li" denotes lithium absorption at 607.82 nm; "g" indicates spectroscopic evidence for a low-gravity atmosphere; "H $\alpha$ " refers to strong, broad $\mathrm{H} \alpha$ emission likely related to accretion/jet events; "d" stands for the presence of a disk.

${ }^{\mathrm{b}}$ There is a $\checkmark$ symbol for those objects located in the $Z J$ eligible zone of cluster members according to our photometric criteria

"Infrared flux excesses ("Y") based on Spitzer (4.5 and $8.0 \mu \mathrm{m})$ and WISE (12.0 $\mu \mathrm{m})$ data. No flux excesses are denoted with "N". Objects off limits of the Spitzer images are indicated with "OL".

References. - (1) Haro \& Moreno (1953); (2) Wiramihardja et al. (1991); (3) Wolk (1996); (4) Reipurth et al. (1998); (5) Béjar et al. (1999); (6) Zapatero Osorio et al. (1999); (7) Zapatero Osorio et al. (2000); (8) Béjar (2001); (9) Béjar et al. (2001); (10) Martín et al. (2001); (11) Barrado y Navascués et al. (2001); (12) Zapatero Osorio et al. (2002b); (13) Barrado y Navascués et al. (2002b); (14) Zapatero Osorio et al. (2002a); (15) Barrado y Navascués et al. (2003); (16) Jayawardhana et al. (2003); (17) McGovern et al. (2004); (18) Andrews et al. (2004); (19) Scholz \& Eislöffel (2004); (20) Béjar et al. (2004b); (21) Caballero et al. (2004); (22) Sherry et al. (2004); (23) Weaver \& Babcock (2004); (24) Kenyon et al. (2005); (25) Burningham et al. (2005); (26) Caballero (2006); (27) Franciosini et al. (2006); (28) Oliveira et al. (2006); (29) Caballero et al. (2006); (30) González-García et al. (2006); (31) Hernández et al. (2007); (32) Caballero et al. (2007); (33) Zapatero Osorio et al. (2007); (34) Scholz \& Jayawardhana (2008); (35) Maxted et al. (2008); (36) Caballero (2008c); (37) Sacco et al. (2008); (38) Luhman et al. (2008); (39) Lodieu et al. (2009); (40) Bihain et al. (2009); (41) Peña Ramírez et al. (2011); (42) Béjar et al. (2011). 
Table 4. VISTA photometry of resolved sources

\begin{tabular}{|c|c|c|c|c|c|c|c|c|c|c|}
\hline$(\mathrm{h}$ & $\begin{array}{c}\alpha \\
\mathrm{m}\end{array}$ & $\begin{array}{ll}\alpha & (\mathrm{J} 20 \\
& \mathrm{s}\end{array}$ & $\begin{array}{l}000) \\
\left({ }^{\circ}\right.\end{array}$ & $\delta$, & $\left.{ }^{\prime \prime}\right)$ & $\begin{array}{c}Z \\
(\mathrm{mag})\end{array}$ & $\begin{array}{c}Y \\
(\mathrm{mag})\end{array}$ & $\begin{array}{c}J \\
(\mathrm{mag})\end{array}$ & $\begin{array}{c}H \\
(\mathrm{mag})\end{array}$ & $\begin{array}{c}K_{s} \\
(\mathrm{mag})\end{array}$ \\
\hline 05 & 38 & 47.45 & -02 & 06 & 45.5 & $22.660 \pm 0.468$ & $\geq 23.1$ & $20.096 \pm 0.088$ & $\geq 19.9$ & $\geq 18.9$ \\
\hline 05 & 40 & 14.23 & -02 & 51 & 46.3 & $22.908 \pm 0.462$ & $\geq 23.1$ & $20.343 \pm 0.107$ & $\geq 19.9$ & $18.7 \overline{69} \pm 0.277$ \\
\hline 05 & 37 & 05.48 & -02 & 25 & 57.9 & $23.170 \pm 0.343$ & $\geq 23.1$ & $20.490 \pm 0.120$ & $19.2 \overline{68} \pm 0.191$ & $18.573 \pm 0.232$ \\
\hline 05 & 40 & 33.21 & -02 & 29 & $54.1^{\mathrm{a}}$ & $14.726 \pm 0.003$ & $13.9 \overline{62} \pm 0.001$ & $13.465 \pm 0.002$ & $12.794 \pm 0.002$ & $12.543 \pm 0.003$ \\
\hline
\end{tabular}

${ }^{\text {a }}$ Extended object found in the $Z J$ search. 
Table 5. Near- and mid-infrared photometry of $\sigma$ Orionis candidate members with youth features

\begin{tabular}{|c|c|c|c|c|c|c|c|c|c|c|c|c|c|c|c|c|}
\hline$\left(\begin{array}{ll}\mathrm{h} & \mathrm{\alpha} \\
\mathrm{h}\end{array}\right.$ & $\begin{array}{l}(\mathrm{J} 2000) \\
\left.{ }^{\mathrm{s}}\right) \quad\left({ }^{\circ}\right.\end{array}$ & $\delta$ & ") & $\begin{array}{c}Z \\
(\mathrm{mag})\end{array}$ & $\begin{array}{c}Y \\
\text { (mag) }\end{array}$ & $\begin{array}{c}J \\
\text { (mag) }\end{array}$ & $\begin{array}{c}H \\
\text { (mag) }\end{array}$ & $\begin{array}{c}K_{s} \\
(\mathrm{mag})\end{array}$ & $\begin{array}{c}{[3.6]} \\
(\mathrm{mag})\end{array}$ & $\begin{array}{c}{[4.5]} \\
\text { (mag) }\end{array}$ & $\begin{array}{c}{[5.8]} \\
(\mathrm{mag})\end{array}$ & $\begin{array}{c}{[8.0]} \\
(\mathrm{mag})\end{array}$ & $\begin{array}{c}W 13.4 \mu \mathrm{m} \\
\quad(\mathrm{mag})\end{array}$ & $\begin{array}{c}W 24.6 \mu \mathrm{m} \\
\quad(\mathrm{mag})\end{array}$ & $\begin{array}{c}W 312.0 \mu \mathrm{m} \\
\quad(\mathrm{mag})\end{array}$ & $\begin{array}{l}W 422.0 \mu \mathrm{m} \\
\quad(\mathrm{mag})\end{array}$ \\
\hline 0538 & $36.88-02$ & & 43.2 & $8 \pm 0$ & $75 \pm$ & $3 \pm$ & $60 \pm$ & $50 \pm 0$ & $76 \pm$ & $5 \pm 0.033$ & $73 \pm$ & $0 \pm$ & $.073 \pm 0$. & $11.799 \pm 0.030$ & & \\
\hline 0539 & $43.18-02$ & 32 & 43.3 & $14.072 \pm 0.002$ & $3.554 \pm 0.001$ & $13.010 \pm 0.001$ & $12.376 \pm 0.001$ & $11.986 \pm 0.002$ & $11.119 \pm 0.029$ & $10.789 \pm 0.029$ & $10.520 \pm 0.029$ & $9.890 \pm 0.029$ & $11.352 \pm 0.025$ & $10.730 \pm 0.023$ & $8.824 \pm 0.032$ & $6.390 \pm 0.070$ \\
\hline 0538 & $21.20-$ & 54 & 11.1 & $14.367 \pm 0.002$ & $13.887 \pm 0.001$ & $13.043 \pm 0.001$ & $12.642 \pm 0.002$ & $12.188 \pm 0.002$ & $11.279 \pm 0.029$ & $11.000 \pm 0.029$ & $10.720 \pm 0.029$ & $10.020 \pm 0.029$ & $11.571 \pm 0.025$ & $11.012 \pm 0.023$ & $8.879 \pm 0.031$ & $6.479 \pm 0.060$ \\
\hline 0538 & $50.78-02$ & & 26.7 & $14.298 \pm 0.002$ & $13.661 \pm 0.002$ & $13.061 \pm 0.001$ & $12.556 \pm 0.002$ & $12.246 \pm 0.002$ & $11.890 \pm 0.029$ & $11.840 \pm 0.029$ & $11.850 \pm 0.029$ & $11.810 \pm 0.039$ & $12.042 \pm 0.037$ & $11.739 \pm 0.040$ & $8.247 \pm 0.181$ & $3.001 \pm 0.132$ \\
\hline 0540 & & & 06.7 & $14.289 \pm 0.002$ & $13.675 \pm 0.001$ & $13.068 \pm 0.001$ & $12.530 \pm 0.002$ & $12.163 \pm 0.002$ & $11.510 \pm 0.029$ & $11.090 \pm 0.029$ & $10.659 \pm 0.029$ & $9.869 \pm 0.029$ & $11.759 \pm 0.026$ & $11.127 \pm 0.022$ & $9.156 \pm 0.037$ & $7.601 \pm 0.150$ \\
\hline 0538 & $17.78-02$ & 40 & 50.1 & $14.276 \pm 0.002$ & $13.671 \pm 0.001$ & $13.086 \pm 0.002$ & $12.588 \pm 0.002$ & $12.228 \pm 0.002$ & $11.770 \pm 0.029$ & $11.520 \pm 0.029$ & $11.319 \pm 0.029$ & $10.670 \pm 0.029$ & $11.948 \pm 0.024$ & $11.529 \pm 0.021$ & $9.798 \pm 0.049$ & $7.607 \pm 0.152$ \\
\hline 0539 & $29.35-02$ & 27 & 21.0 & $14.119 \pm 0.002$ & $13.617 \pm 0.001$ & $13.101 \pm 0.002$ & $12.263 \pm 0.001$ & $11.704 \pm 0.002$ & $10.739 \pm 0.029$ & $10.279 \pm 0.029$ & $9.670 \pm 0.029$ & $8.789 \pm 0.029$ & $10.740 \pm 0.028$ & $9.926 \pm 0.023$ & $7.605 \pm 0.022$ & $5.421 \pm$ \\
\hline 0540 & $01.01-02$ & & 59.8 & $14.337 \pm 0.002$ & $13.677 \pm 0.001$ & $13.103 \pm 0.002$ & $12.602 \pm 0.002$ & $12.269 \pm 0.002$ & $11.909 \pm 0.029$ & $11.810 \pm 0.029$ & $11.829 \pm 0.029$ & $11.600 \pm 0.029$ & $12.093 \pm 0.027$ & $11.830 \pm 0.023$ & & \\
\hline 0538 & $20.50-02$ & 34 & 08.9 & $14.504 \pm 0.003$ & $13.747 \pm 0.001$ & $13.122 \pm 0.002$ & $12.163 \pm 0.001$ & $11.652 \pm 0.002$ & $11.029 \pm 0.029$ & $10.630 \pm 0.029$ & $10.140 \pm 0.029$ & $8.970 \pm 0.029$ & $11.304 \pm 0.025$ & $10.639 \pm 0.022$ & 22 & $583 \pm$ \\
\hline 05 & & & 15.8 & $14.237 \pm 0.002$ & $13.666 \pm 0.001$ & $13.128 \pm 0.002$ & $12.573 \pm 0.002$ & $12.299 \pm 0.002$ & & & & & $12.164 \pm 0.026$ & $11.922 \pm 0.025$ & & \\
\hline 0539 & $26.77-02$ & 42 & 58.3 & $14.103 \pm 0.002$ & $13.612 \pm 0.001$ & $13.144 \pm 0.002$ & $12.410 \pm 0.001$ & $12.133 \pm 0.002$ & $11.840 \pm 0$ & $0 \pm 0.029$ & $9 \pm$ & \pm 0 & $11.989 \pm 0.026$ & $11.683 \pm 0.025$ & $9.982 \pm 0.055$ & \\
\hline 0540 & $23.01-02$ & 36 & 10.1 & $14.125 \pm 0.002$ & $13.680 \pm 0.001$ & $13.153 \pm 0.002$ & $12.560 \pm 0.002$ & $12.293 \pm 0.002$ & & & & & $12.191 \pm 0.025$ & $11.999 \pm 0.025$ & $11.370 \pm 0.173$ & \\
\hline 0538 & & 32 & 43.1 & $1.435 \pm 0.003$ & $13.770 \pm 0.001$ & $13.157 \pm 0.002$ & $12.596 \pm 0.002$ & $12.264 \pm 0.002$ & $11.978 \pm 0.048$ & $11.928 \pm 0.026$ & $11.625 \pm 0$ & $10.584 \pm 0$ & $15.906 \pm 0.118$ & $13.900 \pm 0$ & & \\
\hline 0538 & & & 08.7 & $14.303 \pm 0.002$ & $13.784 \pm 0.001$ & $13.158 \pm 0.002$ & $12.505 \pm 0.002$ & $12.144 \pm 0.002$ & $11.770 \pm$ & $11.619 \pm$ & $11.479 \pm$ & 10. & $11.893 \pm$ & $11.474 \pm$ & 52 & \\
\hline 0538 & $59.22-02$ & 33 & 51.4 & $14.661 \pm 0.003$ & $14.251 \pm 0.001$ & $13.170 \pm 0.002$ & $12.552 \pm 0.002$ & $11.829 \pm 0.002$ & $10.869 \pm 0.029$ & $10.470 \pm 0.029$ & $10.090 \pm 0.029$ & $9.270 \pm 0.029$ & $10.902 \pm 0.023$ & $10.364 \pm 0.022$ & $8.202 \pm 0.025$ & \\
\hline 0538 & $46.85-02$ & 36 & 43.5 & $15.074 \pm 0.004$ & $13.789 \pm 0.002$ & $13.187 \pm 0.002$ & $12.687 \pm 0.002$ & $12.372 \pm 0.002$ & $12.068 \pm 0.036$ & $12.121 \pm 0.038$ & $11.897 \pm 0.025$ & $11.862 \pm 0.043$ & & & & \\
\hline 0538 & & & 23.7 & $14.343 \pm 0.002$ & $13.747 \pm 0.001$ & $13.196 \pm 0.002$ & $12.658 \pm 0.002$ & $69 \pm 0.002$ & & & & & 26 & 25 & & \\
\hline $\begin{array}{l}0539 \\
05\end{array}$ & $15.83-02$ & 36 & 50.7 & $14.244 \pm 0.002$ & $13.711 \pm 0.001$ & $13.200 \pm 0.002$ & $12.576 \pm 0.002$ & $12.221 \pm 0.002$ & $11.560 \pm$ & 1 & 11 & 1 & 11.799 & $11.316 \pm 0.023$ & 9. & \\
\hline 0538 & $08.98-02$ & 20 & 11.0 & $14.223 \pm 0.002$ & $13.748 \pm 0.001$ & $13.200 \pm 0.002$ & $12.595 \pm 0.002$ & $12.371 \pm 0.002$ & $12.137 \pm 0.031$ & $12.199 \pm 0.041$ & $12.016 \pm 0.019$ & $12.021 \pm 0.050$ & $12.242 \pm 0.025$ & $12.046 \pm 0.026$ & $11.924 \pm 0.260$ & \\
\hline 0539 & & 57 & 04.9 & $14.193 \pm 0.002$ & $13.713 \pm 0.001$ & $13.219 \pm 0.002$ & $12.597 \pm 0.002$ & $53 \pm$ & & & & & 25 & & & \\
\hline 0537 & 54.52 & & 26.4 & $14.623 \pm 0.003$ & $13.851 \pm 0.001$ & $13.228 \pm 0.002$ & $12.721 \pm 0.002$ & $12.402 \pm 0.002$ & & & & & $12.212 \pm$ & 11.926 & & \\
\hline 0539 & $57.53-02$ & 32 & 12.1 & $14.371 \pm 0.002$ & $13.805 \pm 0.001$ & $13.231 \pm 0.002$ & $12.734 \pm 0.002$ & $12.413 \pm 0.002$ & $12.136 \pm 0.044$ & $12.110 \pm 0.035$ & $12.032 \pm 0.019$ & $11.968 \pm 0.037$ & $12.276 \pm 0.025$ & $12.048 \pm 0.026$ & \pm 0.254 & \\
\hline 0537 & & & 26.2 & $14.526 \pm 0.003$ & $13.834 \pm 0.001$ & $13.234 \pm 0.002$ & $12.754 \pm 0.002$ & $12.471 \pm 0.003$ & $12.060 \pm 0.029$ & $11.960 \pm 0.029$ & $11.899 \pm 0.029$ & $11.960 \pm 0.039$ & $12.247 \pm 0.026$ & 11.950 .024 & 0.163 & \\
\hline 0538 & & 41 & 31.7 & $417 \pm 0$ & $13830+$ & $55+8$ & $62 \pm 0$ & 570 & 12. & $12.039 \pm$ & 11 & 1 & 26 & & & \\
\hline 0539 & $20.97-02$ & 30 & 33.5 & $14.705 \pm 0.003$ & $13.941 \pm 0.001$ & $13.256 \pm 0.002$ & $12.807 \pm 0.002$ & $12.502 \pm 0.003$ & $12.090 \pm 0.029$ & $11.970 \pm 0.029$ & $11.930 \pm 0.029$ & $11.819 \pm 0.039$ & $12.277 \pm 0.027$ & $11.959 \pm 0.025$ & \pm 0.167 & \\
\hline 0539 & $14.46-02$ & & 33.4 & $14.278 \pm 0.002$ & $13.790 \pm 0.001$ & $13.258 \pm 0.002$ & $12.647 \pm 0.002$ & $12.397 \pm 0.002$ & $12.140 \pm 0.029$ & $12.079 \pm 0.029$ & $12.050 \pm 0.029$ & $12.020 \pm 0.039$ & $12.228 \pm 0.028$ & $12.064 \pm 0.026$ & \pm 0.170 & \\
\hline $05 \quad 39$ & & & $\begin{array}{l}53.4 \\
03.5\end{array}$ & $14.506 \pm 0.003$ & $13.899 \pm 0.001$ & $13.304 \pm 0.002$ & $12.782 \pm 0.002$ & $12.423 \pm 0.002$ & $11.939 \pm 0.029$ & $11.649 \pm 0.029$ & $11.270 \pm \mathrm{c}$ & $10.520 \pm$ & $12.114 \pm 0.027$ & $11.594 \pm 0.024$ & $9.697 \pm 0.048$ & $845 \pm c$ \\
\hline 0538 & 44.4 & 40 & 30.5 & $14.362 \pm 0.002$ & $13.857 \pm 0.001$ & $13.318 \pm 0.002$ & $12.742 \pm 0.002$ & $12.454 \pm 0.003$ & $12.239 \pm 0.029$ & $12.220 \pm 0.029$ & $12.239 \pm 0.039$ & $12.229 \pm 0.070$ & & & & \\
\hline 0539 & $05.24-02$ & 33 & 00.6 & $14.378 \pm 0.002$ & $13.866 \pm 0.001$ & $13.334 \pm 0.002$ & $12.750 \pm 0.002$ & $12.465 \pm 0.003$ & $12.210 \pm 0.029$ & $12.250 \pm 0.029$ & $12.119 \pm 0.029$ & $12.140 \pm 0.039$ & $12.350 \pm 0.026$ & $=0.026$ & 0.264 & \\
\hline & & & & $14.433 \pm 0.003$ & $13.897 \pm 0.001$ & $13.345 \pm 0.002$ & $12.822 \pm 0.002$ & $516 \pm 0.003$ & $12.253 \pm 0.027$ & $12.293 \pm 0.033$ & $12.193 \pm 0$ & $12.124 \pm 0$ & 12 & .026 & & \\
\hline 053 & & 20 & 47.6 & $14.613 \pm 0.003$ & $13.935 \pm 0.001$ & $13.354 \pm 0.002$ & $12.826 \pm 0.002$ & $26 \pm 0.003$ & $11.970 \pm 0.029$ & $11.659 \pm 0.029$ & $11.239 \pm 0.029$ & $10.390 \pm 0.029$ & $12.197 \pm 0.027$ & $11.695 \pm 0.025$ & $9.680 \pm 0.046$ & $4 \pm$ \\
\hline 0537 & $52.11-02$ & 56 & 55.1 & $14.652 \pm 0.003$ & $13.943 \pm 0.001$ & $13.365 \pm 0.002$ & $12.868 \pm 0.002$ & $12.556 \pm 0.003$ & & & & & $12.364 \pm 0.024$ & $12.121 \pm 0.025$ & & \\
\hline 0539 & $26.47-02$ & & 15.5 & $14.277 \pm 0.002$ & $13.847 \pm 0.001$ & $13.386 \pm 0.002$ & $12.706 \pm 0.002$ & $12.472 \pm 0.003$ & $12.279 \pm 0.029$ & $12.239 \pm 0.029$ & $12.210 \pm 0.039$ & $12.159 \pm$ & 12 . & $12.179 \pm 0.026$ & $11.849 \pm 0$ & \\
\hline & & & 25.3 & $14.689 \pm 0.003$ & $14.000 \pm 0.001$ & $13.389 \pm 0.002$ & $12.901 \pm 0.002$ & $12.603 \pm 0.003$ & $12.284 \pm 0$ & $12.248 \pm$ & \pm & $3 \pm$ & $3 \pm 0$ & $12.128 \pm 0.025$ & $\begin{array}{c}1.040 \\
\ldots\end{array}$ & \\
\hline 0538 & 47. & 30 & 37.4 & $14.682 \pm 0.003$ & $14.011 \pm 0.001$ & $13.393 \pm 0.002$ & $12.906 \pm 0$ & $12.561 \pm 0.003$ & & $60 \pm$ & & $12-2 \cdot x-2$ & & 12. & & \\
\hline $05 \quad 39$ & 47.6 & & 23.0 & $14.438 \pm 0.003$ & $13.967 \pm 0.001$ & $17 \pm 0$ & $5 \pm 0$ & $7 \pm 0$ & 12 & 12 r & 12 r & 1 & 12 r & \pm 0.026 & $11.514 \pm 0.197$ & \\
\hline 0538 & 23 & & 14.1 & $14.529 \pm 0.003$ & $13.977 \pm 0.001$ & $13.427 \pm 0.002$ & $12.893 \pm 0.002$ & $12.602 \pm 0.003$ & $12.340 \pm 0.029$ & $12.279 \pm 0.029$ & \pm 0 & \pm 0 & $12.485 \pm 0.027$ & $12.257 \pm 0.027$ & & \\
\hline 0539 & 49 & 23. & $\begin{array}{l}14.1 \\
45.9\end{array}$ & $14.739 \pm 0.003$ & $14.152 \pm 0.001$ & $13.438 \pm 0.002$ & $12.930 \pm 0.002$ & $12.558 \pm 0.003$ & $12.069 \pm 0.029$ & $11.909 \pm 0.029$ & $11.609 \pm 0.029$ & $10.989 \pm 0.029$ & $11.972 \pm 0.024$ & $11.557 \pm 0.023$ & & \\
\hline 0539 & 54 & & & $14.482 \pm 0.003$ & $13.992 \pm 0.001$ & $13.451 \pm 0.002$ & $12.892 \pm 0.002$ & $12.589 \pm 0.003$ & $12.159 \pm$ & $59 \pm$ & 11 & $20 \pm$ & 12. & $8 \pm 0.024$ & \pm 0.028 & $.727 \pm 0$ \\
\hline 053 & 26.3 & & 37.7 & $14.563 \pm 0.003$ & $14.024 \pm 0.001$ & $13.462 \pm 0.0$ & $12.908 \pm 0.002$ & $12.608 \pm 0.003$ & $12.210 \pm 0.029$ & $11.960 \pm 0.029$ & $11.689 \pm 0$ & $10.850 \pm 0$ & \pm 0.028 & $11.803 \pm 0.024$ & $9.930 \pm$ & \\
\hline & 25.0 & & 50.1 & $14.621 \pm 0.003$ & $14.093 \pm 0.001$ & $13.488 \pm 0.002$ & $12.892 \pm 0.002$ & $12.450 \pm 0.002$ & & & & & $11.861 \pm 0.026$ & $11.314 \pm 0.022$ & $9.746 \pm 0.052$ & 7.993 \\
\hline 0538 & 45 & 45 & 23.1 & $14.781 \pm 0.003$ & $14.097 \pm 0.001$ & $13.502 \pm 0.002$ & $13.016 \pm 0.002$ & $12.694 \pm 0.003$ & $12.258 \pm 0.039$ & $12.332 \pm 0.034$ & 12 & & $45 \pm 0.025$ & $12.269 \pm 0.024$ & & \\
\hline 0538 & 16. & & 04.9 & $14.545 \pm 0.003$ & $14.014 \pm 0.001$ & $13.504 \pm 0.002$ & $12.915 \pm 0.002$ & $12.638 \pm 0.003$ & $12.380 \pm 0.029$ & $12.279 \pm 0.029$ & $0 \pm 0$ & $12.250 \pm \mathrm{c}$ & $12.517 \pm 0.026$ & $12.321 \pm 0.028$ & & \\
\hline 0539 & 01. & & 38.8 & $14.520 \pm 0.003$ & $14.017 \pm 0.001$ & $13.506 \pm 0.002$ & $12.926 \pm 0.002$ & $12.676 \pm 0.003$ & $12.460 \pm 0.029$ & $12.359 \pm 0$ & $12.430 \pm 0$ & $12.300 \pm 0$ & $12.540 \pm 0.028$ & $12.317 \pm 0.027$ & & \\
\hline 05 & & & & $14.654 \pm 0.003$ & $14.092 \pm 0.001$ & $13.508 \pm 0.002$ & $12.995 \pm 0.002$ & $12.707 \pm 0.003$ & & & & & $12.492 \pm 0.026$ & $12.257 \pm 0.026$ & & \\
\hline 053 & 54 & & 09.2 & $14.707 \pm 0.003$ & $14.111 \pm 0.001$ & $13.516 \pm 0.002$ & $13.015 \pm 0.002$ & $12.673 \pm 0.003$ & $12.039 \pm$ & 11.800 & 11.449 & 10.81 & $12.344 \pm$ & $11.931 \pm 0.024$ & 66 & 23 \\
\hline 0539 & 04.5 & & 49.2 & $14.604 \pm 0.003$ & $14.000 \pm 0.001$ & \pm 0.002 & \pm 0.001 & $5 \pm 0.002$ & & 10 & & & & $3 \pm 0.023$ & & $6.846 \pm$ \\
\hline 0538 & 17.1 & & & $14.878 \pm 0.003$ & $14.155 \pm 0.001$ & $13.553 \pm 0.002$ & $13.068 \pm 0.002$ & $12.767 \pm 0.003$ & $12.269 \pm 0.034$ & $12.368 \pm 0.038$ & $12.292 \pm 0.024$ & $12.145 \pm 0.046$ & $12.548 \pm 0.027$ & $12.266 \pm 0.026$ & $11.856 \pm 0.23 \mathrm{~s}$ & \\
\hline 05 & & & & $14.617 \pm 0.003$ & $14.115 \pm 0.6$ & $13.559 \pm 0.002$ & $13.003 \pm 0.002$ & $12.725 \pm 0.003$ & & & & & $50 \pm 0$ & $12.423 \pm 0.027$ & & \\
\hline 0539 & $50.56-02$ & 34 & 13.7 & $14.725 \pm 0.003$ & $14.172 \pm 0.001$ & $13.622 \pm 0.002$ & $13.076 \pm 0.002$ & $12.777 \pm 0.003$ & $0 \pm 0.029$ & $00 \pm 0.029$ & $30 \pm 0$ & $90 \pm$ & $12.648 \pm 0.028$ & $12.419 \pm 0.026$ & & \\
\hline
\end{tabular}


Table 5-Continued

\begin{tabular}{|c|c|c|c|c|c|c|c|c|c|c|c|c|c|c|c|c|c|}
\hline$\left(^{\mathrm{h}}\right.$ & $\underset{\mathrm{m}}{\alpha}$ & $\begin{array}{l}(\mathrm{J} 2000) \\
\left.{ }^{\mathrm{s}}\right) \quad\left({ }^{\circ}\right.\end{array}$ & & $\left.{ }^{\prime \prime}\right)$ & $\begin{array}{c}Z \\
(\mathrm{mag})\end{array}$ & $\begin{array}{c}Y \\
(\mathrm{mag})\end{array}$ & $\begin{array}{c}J \\
(\mathrm{mag})\end{array}$ & $\begin{array}{c}H \\
(\mathrm{mag})\end{array}$ & $\begin{array}{c}K_{s} \\
(\mathrm{mag})\end{array}$ & $\begin{array}{c}{[3.6]} \\
(\mathrm{mag})\end{array}$ & $\begin{array}{c}{[4.5]} \\
(\mathrm{mag})\end{array}$ & $\begin{array}{c}{[5.8]} \\
(\mathrm{mag})\end{array}$ & $\begin{array}{c}{[8.0]} \\
(\mathrm{mag})\end{array}$ & $\begin{array}{c}W 13.4 \mu \mathrm{m} \\
(\mathrm{mag})\end{array}$ & $\begin{array}{c}W 24.6 \mu \mathrm{m} \\
(\mathrm{mag})\end{array}$ & $\begin{array}{c}W 312.0 \mu \mathrm{m} \\
(\mathrm{mag})\end{array}$ & $\begin{array}{c}W 422.0 \mu \mathrm{m} \\
(\mathrm{mag})\end{array}$ \\
\hline & & & & .8 & 003 & 001 & 002 & \pm 0.002 & \pm 0.003 & & & & & $12.538 \pm 0.049$ & $2.282 \pm 0.043$ & & \\
\hline 05 & & $40.08-02$ & & 37.0 & $14.817 \pm 0.003$ & $14.219 \pm 0.001$ & $13.651 \pm 0.002$ & $13.126 \pm 0.002$ & $12.812 \pm 0.003$ & $12.520 \pm 0.029$ & $12.460 \pm 0.029$ & $12.430 \pm 0.039$ & $12.420 \pm 0.039$ & $12.657 \pm 0.027$ & $12.424 \pm 0.027$ & & \\
\hline 05 & & $34.39-02$ & & 09.5 & $14.935 \pm 0.003$ & $14.260 \pm 0.001$ & $13.664 \pm 0.002$ & $13.161 \pm 0.002$ & $12.821 \pm 0.003$ & & & & & $12.553 \pm 0.028$ & $12.112 \pm 0.027$ & $10.156 \pm 0.069$ & \\
\hline 05 & 38 & $23.34-02$ & 25 & 34.6 & $14.902 \pm 0.003$ & $14.440 \pm 0.001$ & $13.664 \pm 0.002$ & $13.080 \pm 0.002$ & $12.562 \pm 0.003$ & $11.899 \pm 0.029$ & $11.579 \pm 0.029$ & $11.220 \pm 0.029$ & $0.529 \pm$ & $11.761 \pm 0.024$ & $11.290 \pm 0.023$ & $9.672 \pm$ & 1 \\
\hline 05 & & $39.72-02$ & 40 & 19.7 & $14.848 \pm 0.003$ & $14.241 \pm 0.001$ & $13.688 \pm 0.002$ & $13.162 \pm 0.002$ & $12.859 \pm 0.003$ & $12.533 \pm 0.038$ & $12.702 \pm 0.045$ & $12.509 \pm 0.031$ & $12.465 \pm 0.093$ & $12.715 \pm 0.025$ & $12.468 \pm 0.026$ & & \\
\hline 05 & & $54.93-02$ & & 58.3 & $14.860 \pm 0.003$ & $14.301 \pm 0.001$ & $13.725 \pm 0.002$ & $13.190 \pm 0.002$ & $12.890 \pm 0.003$ & $12.600 \pm 0.029$ & $12.550 \pm 0.029$ & $12.470 \pm 0.039$ & $12.479 \pm 0.050$ & $12.797 \pm 0.027$ & $12.550 \pm 0.029$ & $11.959 \pm 0.259$ & \\
\hline 05 & & $23.07-02$ & 36 & 49.4 & $14.955 \pm 0.003$ & $14.353 \pm 0.001$ & $13.726 \pm 0.002$ & $13.197 \pm 0.002$ & $12.809 \pm 0.003$ & $12.220 \pm 0.029$ & $11.800 \pm 0.029$ & $11.300 \pm 0.050$ & $10.760 \pm 0.039$ & $12.290 \pm 0.025$ & $11.684 \pm 0.023$ & $9.530 \pm 0.096$ & $6.580 \pm 0.110$ \\
\hline 05 & & & & 08.1 & $14.920 \pm 0.003$ & $14.304 \pm 0.001$ & $13.736 \pm 0.002$ & $13.196 \pm 0.002$ & $12.898 \pm 0.003$ & $12.399 \pm 0.029$ & $12.079 \pm 0.029$ & $11.659 \pm 0.029$ & $10.930 \pm 0.029$ & $12.598 \pm 0.027$ & $12.076 \pm 0.025$ & $10.347 \pm 0.073$ & $8.263 \pm 0.267$ \\
\hline 05 & & $08.22-02$ & & 28.4 & $15.026 \pm 0.004$ & $14.346 \pm 0.001$ & $13.768 \pm 0.002$ & $13.264 \pm 0.002$ & $12.947 \pm 0.003$ & $12.615 \pm 0.042$ & $12.573 \pm 0.052$ & $12.617 \pm 0.037$ & $12.495 \pm 0.061$ & $12.788 \pm 0.030$ & $12.504 \pm 0.031$ & & \\
\hline 05 & & $50.60-02$ & 42 & 42.9 & $15.086 \pm 0.004$ & $14.403 \pm 0.001$ & $13.789 \pm 0.002$ & $13.301 \pm 0.002$ & $12.953 \pm 0.003$ & $12.439 \pm 0.029$ & $12.149 \pm 0.029$ & $11.710 \pm 0.029$ & $11.029 \pm 0.029$ & $12.637 \pm 0.026$ & $12.078 \pm 0.025$ & $9.858 \pm 0$ & \\
\hline 05 & 39 & & 13 & 33.4 & $14.943 \pm 0.003$ & $14.373 \pm 0.001$ & $13.831 \pm 0.002$ & $13.303 \pm 0.002$ & $12.999 \pm 0.003$ & & & & & $12.868 \pm 0.027$ & $12.648 \pm 0.028$ & & \\
\hline 05 & & 56.14 & & 26.6 & $15.020 \pm 0.004$ & $14.405 \pm 0.001$ & $13.865 \pm 0.002$ & $13.315 \pm 0.002$ & $13.043 \pm 0.004$ & $12.753 \pm 0.029$ & & $\ldots$ & . & $12.857 \pm 0.028$ & $12.610 \pm 0.029$ & . & $\ldots$ \\
\hline 05 & 38 & $35.29-02$ & 33 & 13.1 & $15.230 \pm 0.004$ & $14.527 \pm 0.001$ & $13.883 \pm 0.002$ & $13.422 \pm 0.003$ & $13.072 \pm 0.004$ & $12.802 \pm 0.037$ & $12.713 \pm 0.046$ & $12.513 \pm 0.038$ & $12.468 \pm 0.057$ & & & & \\
\hline 05 & 40 & $05.25-02$ & 30 & $\begin{array}{l}52.4 \\
52.4\end{array}$ & $15.139 \pm 0.004$ & $14.526 \pm 0.001$ & $13.908 \pm 0.002$ & $13.402 \pm 0.003$ & $13.033 \pm 0.004$ & $12.409 \pm 0.029$ & $12.130 \pm 0.029$ & $11.770 \pm 0.029$ & $11.239 \pm 0.029$ & $12.780 \pm 0.027$ & $12.329 \pm 0.026$ & $10.382 \pm 0.076$ & $8.119 \pm c$ \\
\hline 05 & & & 44 & 00.8 & $15.343 \pm 0.004$ & $14.637 \pm 0.001$ & $14.009 \pm 0.003$ & $13.523 \pm 0.003$ & $13.154 \pm 0.004$ & $12.505 \pm 0.025$ & $12.475 \pm 0.046$ & $11.932 \pm 0.028$ & $11.145 \pm 0.027$ & $12.772 \pm 0.026$ & $12.224 \pm 0.023$ & $10.464 \pm 0.074$ & \\
\hline 05 & & $26.84-02$ & 38 & 46.1 & $15.371 \pm 0.004$ & $14.638 \pm 0.001$ & $14.048 \pm 0.003$ & $13.536 \pm 0.003$ & $13.173 \pm 0.004$ & $12.569 \pm 0.029$ & $12.270 \pm 0.029$ & $11.989 \pm 0.029$ & $11.289 \pm 0.029$ & $12.901 \pm 0.026$ & $12.406 \pm 0.026$ & $10.322 \pm 0.071$ & \\
\hline 05 & 38 & $13.21-02$ & 24 & 07.6 & $15.361 \pm 0.004$ & $14.666 \pm 0.001$ & $14.058 \pm 0.003$ & $13.572 \pm 0.003$ & $13.276 \pm 0.004$ & $12.817 \pm 0.032$ & $12.910 \pm 0.040$ & $12.724 \pm 0.032$ & $12.736 \pm 0.065$ & $13.061 \pm 0.028$ & $12.760 \pm 0.029$ & & \\
\hline 05 & $40 \mathrm{C}$ & & 51 & 59.4 & $15.587 \pm 0.005$ & $14.757 \pm 0.001$ & $14.063 \pm 0.003$ & $13.638 \pm 0.003$ & $13.243 \pm 0.004$ & $12.847 \pm 0.028$ & $12.979 \pm 0.050$ & $12.710 \pm 0.030$ & $12.616 \pm 0.067$ & $13.007 \pm 0.027$ & $12.690 \pm 0.029$ & & \\
\hline 05 & & 08. & & 44.8 & $15.212 \pm 0.004$ & $14.632 \pm 0.001$ & $14.069 \pm 0.003$ & $13.544 \pm 0.003$ & $13.268 \pm 0.004$ & $12.899 \pm 0.029$ & $12.760 \pm 0.029$ & $12.590 \pm 0.039$ & $12.550 \pm 0.039$ & $13.040 \pm 0.027$ & $12.724 \pm 0.029$ & & \\
\hline 05 & 39 & $44.51-02$ & 24 & 43.2 & $15.418 \pm 0.004$ & $14.701 \pm 0.001$ & $14.104 \pm 0.003$ & $13.572 \pm 0.003$ & $13.232 \pm 0.004$ & $12.630 \pm 0.029$ & $12.350 \pm 0.029$ & $12.039 \pm 0.029$ & $11.289 \pm 0.029$ & $12.795 \pm 0.027$ & $12.284 \pm 0.027$ & $10.197 \pm$ & \\
\hline 05 & 38 & $16.99-02$ & 14 & 46.4 & $15.357 \pm 0.004$ & $14.696 \pm 0.001$ & $14.143 \pm 0.003$ & $13.613 \pm 0.003$ & $13.332 \pm 0.004$ & & & & & $13.202 \pm 0.031$ & $12.910 \pm 0.032$ & & \\
\hline & & & & 44.5 & $15.503 \pm 0.005$ & $14.777 \pm 0.001$ & $14.172 \pm 0.003$ & $13.690 \pm 0.003$ & $13.384 \pm 0.004$ & מ & 9 & 9 & 1 & & & & \\
\hline 05 & 38 & $33.88-02$ & 45 & 07.8 & $15.382 \pm 0.004$ & $14.749 \pm 0.001$ & $14.177 \pm 0.003$ & $13.673 \pm 0.003$ & $13.320 \pm 0.004$ & $12.817 \pm 0.028$ & $12.739 \pm 0.043$ & $12.378 \pm 0.024$ & $11.477 \pm 0.024$ & $12.992 \pm$ & 1 & $\ldots$ & $\ldots$ \\
\hline 05 & 39 & $44.33-02$ & 33 & 02.8 & $15.616 \pm 0.005$ & $14.875 \pm 0.002$ & $14.257 \pm 0.003$ & $13.785 \pm 0.003$ & $13.438 \pm 0.004$ & $13.239 \pm 0.043$ & $13.180 \pm 0.053$ & $12.990 \pm 0.039$ & $12.869 \pm 0.075$ & $13.257 \pm 0.029$ & $12.945 \pm 0.031$ & & \\
\hline 05 & & & 44 & 30.5 & $15.696 \pm 0.005$ & $14.916 \pm 0.002$ & $14.281 \pm 0.003$ & $13.826 \pm 0.003$ & $13.453 \pm 0.005$ & $13.029 \pm 0.037$ & $13.015 \pm 0.045$ & $12.897 \pm 0$ & $12.906 \pm 0$ & $13.265 \pm 0.033$ & $12.933 \pm 0.033$ & & \\
\hline 05 & & $10.12-02$ & 54 & 50.7 & $15.644 \pm 0.005$ & $14.929 \pm 0.002$ & $14.311 \pm 0.003$ & $13.833 \pm 0.003$ & $13.481 \pm 0.005$ & $13.069 \pm 0.029$ & $12.970 \pm 0.029$ & $12.960 \pm 0.039$ & $12.899 \pm 0.059$ & $13.219 \pm 0.028$ & $12.930 \pm 0.031$ & $\ldots$ & \\
\hline 05 & 38 & $36.24-02$ & 08 & 24.2 & $15.591 \pm 0.005$ & $14.899 \pm 0.002$ & $14.312 \pm 0.003$ & $13.806 \pm 0.003$ & $13.486 \pm 0.005$ & & & & & $13.319 \pm 0.030$ & $13.051 \pm 0.033$ & & \\
\hline 05 & & 48.1 & & 53.6 & $15.718 \pm 0.005$ & $15.008 \pm 0.002$ & $14.374 \pm 0.003$ & $13.900 \pm 0.003$ & $13.531 \pm 0.005$ & $13.000 \pm 0.029$ & $12.720 \pm 0.029$ & $12.369 \pm 0.039$ & $11.550 \pm 0.039$ & $13.215 \pm 0.027$ & $12.677 \pm 0.028$ & $10.746 \pm 0.095$ & \\
\hline & 39 & & 33 & 32.8 & $15.718 \pm 0.005$ & $15.004 \pm 0.002$ & $14.409 \pm 0.003$ & $13.917 \pm 0.004$ & $13.580 \pm 0.005$ & $13.296 \pm 0.028$ & $13.203 \pm 0.030$ & $13.095 \pm$ & $13.022 \pm 0.087$ & $9 \pm$ & $13.121 \pm$ & & \\
\hline 05 & 38 & $47.15-02$ & 57 & 55.7 & $16.064 \pm 0.006$ & $15.142 \pm 0.002$ & $14.453 \pm 0.003$ & $13.985 \pm 0.004$ & $13.594 \pm 0.005$ & & & & $11.541 \pm 0.057$ & $13.133 \pm 0.026$ & $12.554 \pm 0.029$ & $10.830 \pm$ & . \\
\hline 05 & & $49.28-02$ & 23 & 57.6 & $15.771 \pm 0.005$ & $15.167 \pm 0.002$ & $14.463 \pm 0.003$ & $13.906 \pm 0.004$ & $13.453 \pm 0.005$ & $12.789 \pm 0.029$ & $12.470 \pm 0.029$ & $12.010 \pm 0.029$ & $11.569 \pm 0.039$ & $12.799 \pm 0.027$ & $12.242 \pm 0.025$ & $10.567 \pm 0.086$ & \\
\hline & & 38.5 & 41 & 55.8 & $15.723 \pm 0.005$ & $15.093 \pm 0.002$ & $14.476 \pm 0.003$ & $13.994 \pm 0.004$ & $13.652 \pm 0.005$ & $13.345 \pm 0.032$ & $13.293 \pm 0.054$ & $13.411 \pm 0.056$ & $13.189 \pm 0.107$ & $13.502 \pm 0.029$ & $13.205 \pm 0.032$ & 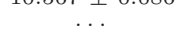 & \\
\hline 05 & 40 & 13.9 : & 31 & 27.4 & $16.275 \pm 0.007$ & $15.259 \pm 0.002$ & $14.482 \pm 0.003$ & $14.002 \pm 0.004$ & $13.585 \pm 0.005$ & & & & & $13.310 \pm 0.030$ & $12.972 \pm 0.033$ & & \\
\hline 05 & & $13.31-02$ & & 33.0 & $15.850 \pm 0.005$ & $15.123 \pm 0.002$ & $14.506 \pm 0.003$ & $14.030 \pm 0.004$ & $13.694 \pm 0.005$ & $13.310 \pm 0.029$ & $13.189 \pm 0.029$ & $13.069 \pm 0.039$ & $13.159 \pm 0.059$ & $13.535 \pm 0.030$ & $13.279 \pm 0.035$ & & \\
\hline 0 & & 25.68 & & 21.6 & $15.893+0.006$ & $15.198+0.002$ & $14.593+0.004$ & $14.092+0.004$ & $13.751+0.005$ & $13.380=0.029$ & $13.319+0.029$ & $13.310 \pm 0.050$ & $13.310+0.070$ & $13.409+0.027$ & $13.189+0.033$ & & \\
\hline 05 & 38 & 35. & 25 & 22.2 & $16.058 \pm 0.006$ & $15.269 \pm 0.002$ & $14.593 \pm 0.004$ & $14.126 \pm 0.004$ & $13.757 \pm 0.005$ & $13.340 \pm 0.029$ & $13.229 \pm 0.029$ & $13.250 \pm 0.050$ & $13.189 \pm 0.070$ & $13.493 \pm 0.028$ & $13.163 \pm$ & & \\
\hline 05 & 40 & $32.49-02$ & 40 & 59.8 & $16.089 \pm 0.006$ & $15.273 \pm 0.002$ & $14.630 \pm 0.004$ & $14.143 \pm 0.004$ & $13.763 \pm 0.005$ & & & & & $13.524 \pm 0.030$ & $13.241 \pm 0.035$ & & \\
\hline 05 & & 08.95 & & 57.9 & $16.324 \pm 0.007$ & $15.370 \pm 0.002$ & $14.661 \pm 0.004$ & $14.199 \pm 0.004$ & $312 \pm 0.006$ & $13.326 \pm 0.048$ & $13.340 \pm 0.0$ & $13.352 \pm 0.056$ & $13.131 \pm 0.101$ & $75 \pm 0.028$ & $13.143 \pm 0.033$ & & \\
\hline & & 34.3 & & 46.9 & $16.258 \pm 0.007$ & $15.345 \pm 0.002$ & $14.690 \pm 0.004$ & $14.220 \pm 0.004$ & $13.858 \pm 0.006$ & $13.390 \pm 0.029$ & $13.270 \pm 0.029$ & $13.250 \pm 0.039$ & $13.100 \pm 0.059$ & $13.639 \pm 0.028$ & $13.308 \pm 0.038$ & & \\
\hline & 39 & 54.3 & 37 & 18.9 & $16.155 \pm 0.006$ & $15.360 \pm 0.002$ & $14.693 \pm 0.004$ & $14.221 \pm 0.004$ & $13.843 \pm 0.006$ & $78 \pm 0$ & $2 \pm$ & $92 \pm$ & $13.300 \pm$ & $13.649 \pm 0$ & $13.338 \pm$ & & \\
\hline & & 44. & & & $16.323 \pm 0$ & $15.414 \pm 0$ & $9 \pm 0$ & $14.274 \pm 0$ & $9 \pm 0$ & $7 \pm 0$ & $8 \pm$ & $16 \pm 0$ & $12.998 \pm 0.164$ & & & & \\
\hline & & 04.4 & & 35.3 & $16.094 \pm 0.006$ & $15.317 \pm 0.002$ & $14.715 \pm 0.004$ & $14.210 \pm 0.004$ & $13.890 \pm 0.006$ & $13.529 \pm 0.029$ & $13.359 \pm 0.029$ & $13.300 \pm 0.050$ & $13.130 \pm 0.059$ & $55 \pm 0$ & $13.263 \pm$ & & \\
\hline & & 01.9 & & 02.9 & $15.909 \pm 0.006$ & $15.365 \pm 0.002$ & $14.736 \pm 0.004$ & $13.599 \pm 0.003$ & $12.838 \pm 0.003$ & $11.472 \pm 0.019$ & $11.097 \pm 0.026$ & $10.593 \pm 0.014$ & $9.839 \pm 0.029$ & $11.787 \pm 0.025$ & $11.003 \pm 0.022$ & $8.921 \pm$ & \\
\hline 05 & & 26.2 & 40 & & $16.180 \pm 0.006$ & $15.408 \pm 0.002$ & $14.758 \pm 0.004$ & $14.322 \pm 0.004$ & $13.951 \pm 0.006$ & $13.623 \pm 0.032$ & $13.546 \pm 0.050$ & $13.411 \pm 0.057$ & $13.423 \pm 0.112$ & $13.710 \pm 0$ & $13.365 \pm 0.033$ & & \\
\hline & 38 & $20.90-02$ & 51 & 28.0 & $16.246 \pm 0.007$ & $15.420 \pm 0.002$ & $14.767 \pm 0.004$ & $14.311 \pm 0.004$ & $13.934 \pm 0.006$ & $13.387 \pm 0.045$ & $13.425 \pm 0.040$ & $13.281 \pm 0$ & $13.349 \pm 0.110$ & $13.689 \pm 0.029$ & $13.385 \pm 0.036$ & & \\
\hline & & $28.97-02$ & & 47.2 & $16.219 \pm 0.006$ & $15.438 \pm 0.002$ & $14.778 \pm 0.004$ & $14.298 \pm 0.004$ & $13.888 \pm 0.006$ & $13.173 \pm 0.035$ & $12.958 \pm 0.035$ & $12.539 \pm 0.030$ & $11.906 \pm 0.048$ & $13.428 \pm 0.029$ & $12.874 \pm 0.031$ & & $394 \pm$ \\
\hline & & & & & 16.2 & & & $14.326 \pm$ & $55 \pm$ & & & & & & & & \\
\hline & & 17. & & 24.2 & $16.291 \pm 0.007$ & $15.473 \pm 0.002$ & $14.811 \pm 0.004$ & $14.373 \pm 0.005$ & $13.980 \pm 0.006$ & $13.619 \pm 0.052$ & $24 \pm 0.041$ & $13.527 \pm 0.061$ & $35 \pm 0$ & $13.793 \pm 0$ & $13.420 \pm 0.035$ & & \\
\hline & & 25.4 & & & $16.410 \pm 0.007$ & $15.575 \pm 0.002$ & $14.890 \pm 0.004$ & $14.286 \pm 0.004$ & $13.737 \pm 0.005$ & $12.730 \pm 0.032$ & $12.487 \pm 0.035$ & $12.005 \pm 0.017$ & $11.418 \pm 0.041$ & $13.026 \pm 0.027$ & $12.436 \pm 0.026$ & 10.503 & \\
\hline 05 & 39 & $07.61-02$ & 29 & & $16.272 \pm 0.007$ & $15.550 \pm 0.002$ & $14.902 \pm 0.004$ & $14.327 \pm 0.004$ & $13.987 \pm 0.006$ & $13.539 \pm 0.055$ & $13.596 \pm 0.047$ & $13.433 \pm 0.054$ & $13.572 \pm 0.161$ & $13.728 \pm 0.030$ & $13.511 \pm 0.038$ & & \\
\hline
\end{tabular}


Table 5-Continued

\begin{tabular}{|c|c|c|c|c|c|c|c|c|c|c|c|c|c|c|c|c|}
\hline $\begin{array}{cc} & \alpha \\
\left(\begin{array}{ll}\mathrm{h} & \mathrm{m} \\
\end{array}\right.\end{array}$ & $\begin{array}{l}(\mathrm{J} 200 \\
\mathrm{s})\end{array}$ & $\begin{array}{l}{ }^{\circ} \\
{ }^{\circ}\end{array}$ & $\begin{array}{ll}\delta & \\
& \prime \prime\end{array}$ & $\begin{array}{c}Z \\
(\mathrm{mag}) \\
\end{array}$ & $\begin{array}{c}Y \\
(\mathrm{mag})\end{array}$ & $\begin{array}{c}J \\
(\mathrm{mag})\end{array}$ & $\begin{array}{c}H \\
(\mathrm{mag}) \\
\end{array}$ & $\begin{array}{c}K_{s} \\
(\mathrm{mag})\end{array}$ & $\begin{array}{c}{[3.6]} \\
(\mathrm{mag}) \\
\end{array}$ & $\begin{array}{c}{[4.5]} \\
(\mathrm{mag}) \\
\end{array}$ & $\begin{array}{c}{[5.8]} \\
(\mathrm{mag}) \\
\end{array}$ & $\begin{array}{c}{[8.0]} \\
(\mathrm{mag}) \\
\end{array}$ & $\begin{array}{c}W 13.4 \mu \mathrm{m} \\
(\mathrm{mag})\end{array}$ & $\begin{array}{l}W 24.6 \mu \mathrm{m} \\
\quad(\mathrm{mag})\end{array}$ & $\begin{array}{c}W 312.0 \mu \mathrm{m} \\
\quad(\mathrm{mag})\end{array}$ & $\begin{array}{c}W 422.0 \mu \mathrm{m} \\
\quad(\mathrm{mag})\end{array}$ \\
\hline
\end{tabular}

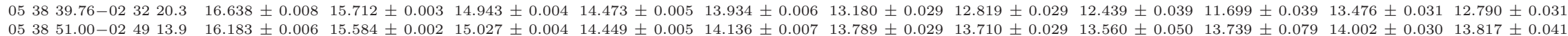

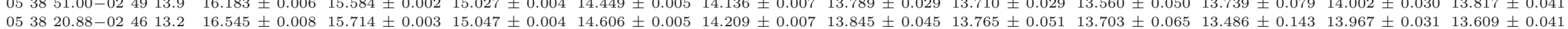

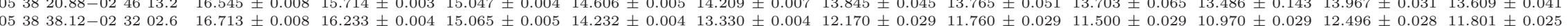

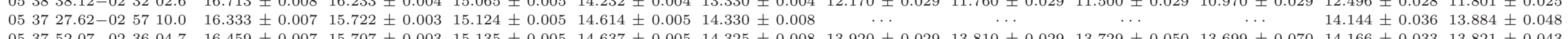

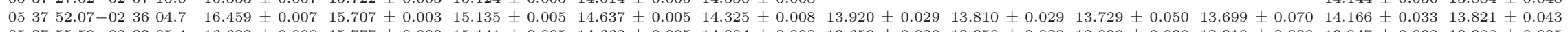

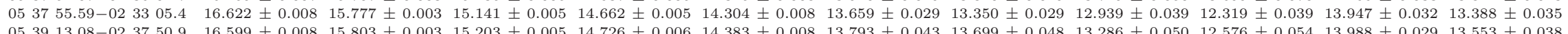

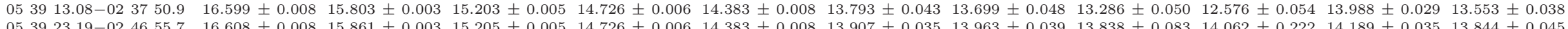

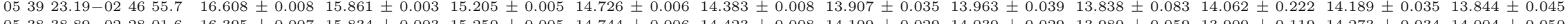

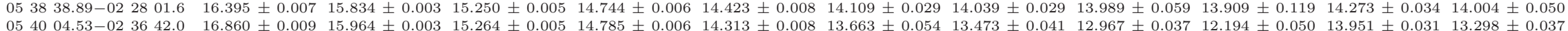

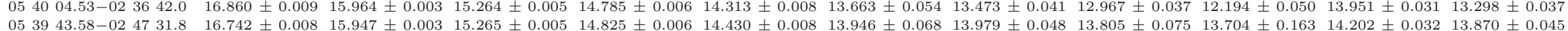

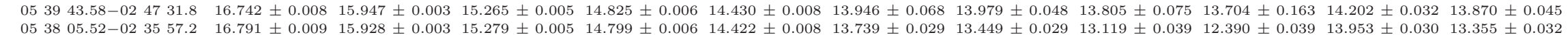

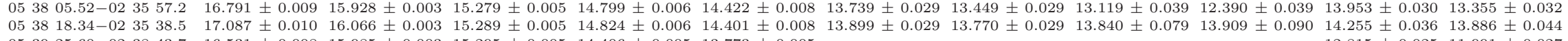

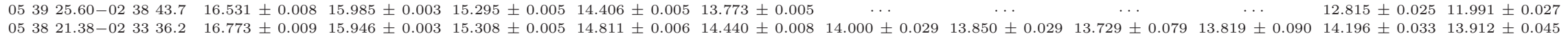

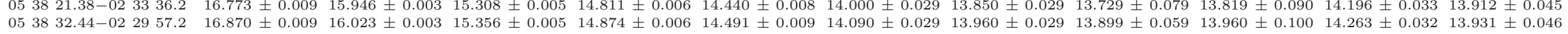

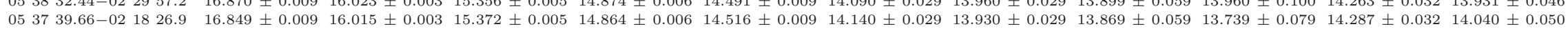

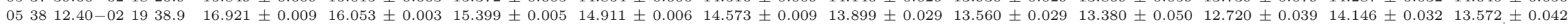

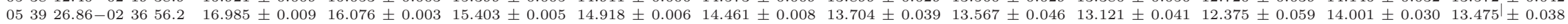

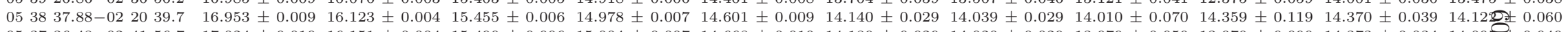

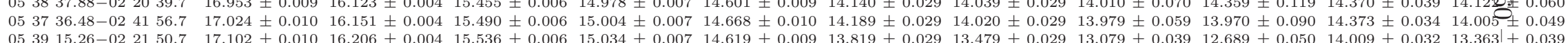
14.408 $\pm 0.03413 .722 \pm 0.042$

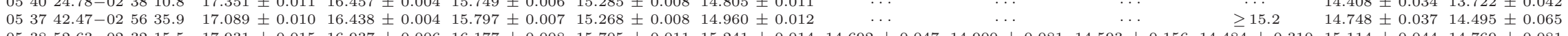

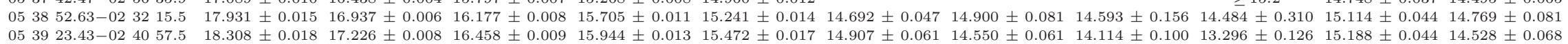

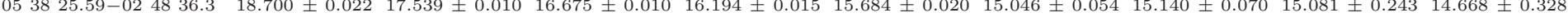

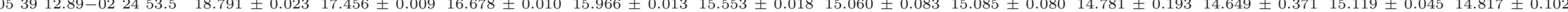

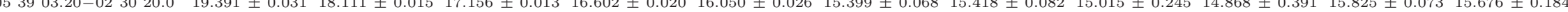

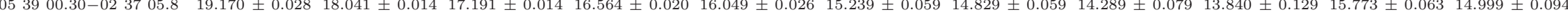

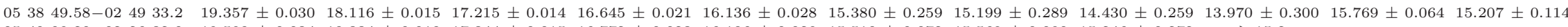
$\begin{array}{lllllllllllllllll}05 & 40 & 09.20-02 & 26 & 32.0 & 19.598 \pm 0.034 & 18.334 \pm 0.018 & 17.344 \pm 0.015 & 16.772 \pm 0.023 & 16.186 \pm 0.029 & 15.510 \pm 0.072 & 15.569 \pm 0.090 & 15.246 \pm 0.272 & \geq 15.2\end{array}$

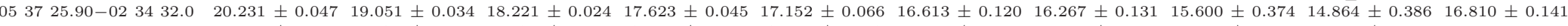

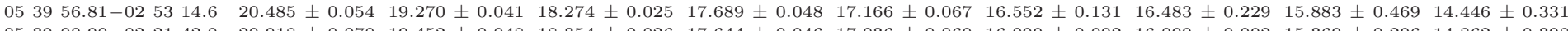

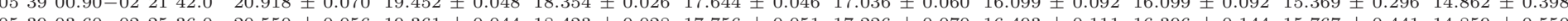

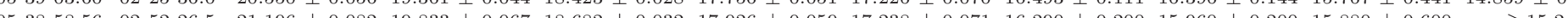

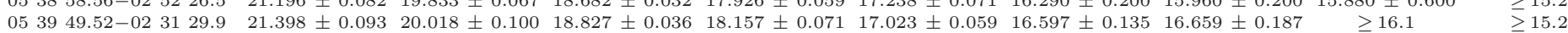

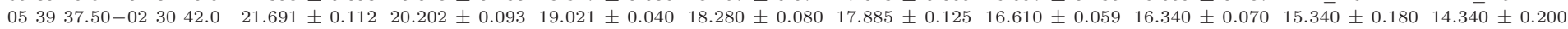
$\begin{array}{llllllllllllllllllll}0539 & 42.05-02 & 30 & 31.6 & 21.775 & \pm 0.118 & 20.340 \pm 0.106 & 19.143 \pm 0.044 & 18.556 \pm 0.101 & 17.889 & \pm 0.126 & 16.980 \pm 0.157 & 16.831 \pm 0.241 & \geq 16.1 & \geq 15.2\end{array}$

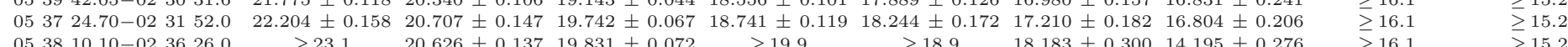

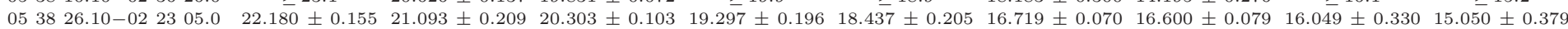

Note. - No WISE photometry implies quoted signal-to-noise ratio below 4. 
Table 6. Near- and mid-infrared photometry of known $\sigma$ Orionis photometric candidates

\begin{tabular}{|c|c|c|c|c|c|c|c|c|c|c|c|c|c|c|c|c|}
\hline $\mathrm{h}$ & & $\begin{array}{l}(\mathrm{J} 2000) \\
\mathrm{s}) \quad\left({ }^{\circ}\right.\end{array}$ & $\delta$, & $\begin{array}{c}Z \\
(\mathrm{mag})\end{array}$ & $\begin{array}{c}Y \\
(\mathrm{mag})\end{array}$ & $\begin{array}{c}J \\
(\mathrm{mag})\end{array}$ & $\begin{array}{c}H \\
(\mathrm{mag})\end{array}$ & $\begin{array}{c}K_{s} \\
(\mathrm{mag})\end{array}$ & $\begin{array}{c}{[3.6]} \\
(\mathrm{mag})\end{array}$ & $\begin{array}{l}{[4.5]} \\
(\mathrm{mag})\end{array}$ & $\begin{array}{c}{[5.8]} \\
(\mathrm{mag})\end{array}$ & $\begin{array}{c}{[8.0]} \\
(\mathrm{mag})\end{array}$ & $\begin{array}{c}W 13.4 \mu \mathrm{m} \\
(\mathrm{mag})\end{array}$ & $\begin{array}{l}W 24.6 \mu \mathrm{m} \\
\quad(\mathrm{mag})\end{array}$ & $\begin{array}{c}W 312.0 \mu \mathrm{m} \\
\quad(\mathrm{mag})\end{array}$ & $\begin{array}{c}W 422.0 \mu \mathrm{m} \\
\quad(\mathrm{mag})\end{array}$ \\
\hline & & & & & & & & & 8 & & $770 \pm$ & $702 \pm$ & $.768 \pm 0.024$ & $10.569 \pm 0.022$ & $0.338 \pm 0.073$ & \\
\hline & & $41.45-02$ & 2907. & $324 \pm 0.002$ & $607 \pm 0.001$ & $13.017 \pm 0.001$ & $12.492 \pm 0.002$ & $12.188 \pm 0.002$ & & ... & $\ldots$ & 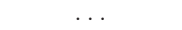 & $11.951 \pm 0.025$ & $11.686 \pm 0.023$ & $\ldots$ & \\
\hline & & $25.61-02$ & 3404. & $14.288 \pm 0.002$ & $13.664 \pm 0.001$ & $13.118 \pm 0.002$ & $12.598 \pm 0.002$ & $12.286 \pm 0.002$ & $11.890 \pm 0.031$ & $12.024 \pm 0.031$ & $816 \pm 0$ & $11.737 \pm 0.041$ & & & & \\
\hline 53 & 38 & $28.43-03$ & 0025. & $14.260 \pm 0.002$ & $3.694 \pm 0.001$ & $13.125 \pm 0.002$ & $12.612 \pm 0.002$ & $12.314 \pm 0.002$ & $11.966 \pm 0.039$ & $12.001 \pm 0.043$ & & $11.845 \pm 0.050$ & $12.056 \pm 0.025$ & $11.848 \pm 0.025$ & & \\
\hline 53 & 37 & $00.30-02$ & 2826 . & $14.280 \pm 0.002$ & $.755 \pm 0.001$ & $13.241 \pm 0.002$ & $12.653 \pm 0.002$ & $12.391 \pm 0.002$ & & & .. & 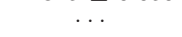 & $12.261 \pm 0.026$ & $12.001 \pm 0.024$ & $9.859 \pm 0.057$ & $.056 \pm 0$ \\
\hline & & $49.28-02$ & 4354. & $14.269 \pm 0.002$ & $13.814 \pm 0.001$ & $13.267 \pm 0.002$ & $12.677 \pm 0.002$ & $12.382 \pm 0.002$ & & & & & $12.148 \pm 0.025$ & $11.796 \pm 0.024$ & $10.175 \pm 0.056$ & \\
\hline 3 & 37 & $35.14-02$ & 2657. & $.650 \pm 0.003$ & $888 \pm 0.001$ & $13.319 \pm 0.002$ & $12.785 \pm 0.002$ & $12.479 \pm 0.003$ & $12.052 \pm 0.036$ & $12.065 \pm 0.025$ & $11.993 \pm 0.022$ & $934 \pm 0.041$ & $12.326 \pm 0.027$ & $12.015 \pm 0.025$ & & \\
\hline 53 & & 13.47 & 3739. & $482 \pm 0.003$ & $901 \pm 0.001$ & $13.358 \pm 0.002$ & $12.827 \pm 0.002$ & $12.550 \pm 0.003$ & $12.188 \pm$ & $12.174=$ & $12.036=$ & $12.080=$ & $12.343 \pm 0.024$ & $12.121 \pm 0.025$ & $11.496 \pm 0.187$ & . \\
\hline 53 & & $47.50-02$ & 3038. & $14.682 \pm 0.003$ & $14.011 \pm 0.001$ & $13.393 \pm 0.002$ & $12.906 \pm 0.002$ & $12.561 \pm 0.003$ & $12.279 \pm 0$ & $12.215 \pm 0.035$ & $12.089 \pm 0.022$ & $12.201 \pm 0.054$ & $\ldots$ & -5 & & 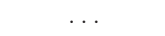 \\
\hline 553 & 38 & $52.53-02$ & 1221. & $14.410 \pm 0.003$ & $13.939 \pm 0.001$ & $13.396 \pm 0.002$ & $12.844 \pm 0.002$ & $12.565 \pm 0.003$ & & $-5+2>$ & & & 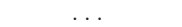 & & & 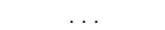 \\
\hline 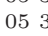 & 37 & $11.68-02$ & 3156. & $14.545 \pm 0.003$ & $13.966 \pm 0.001$ & $13.443 \pm 0.002$ & $12.875 \pm 0.002$ & $12.605 \pm 0.003$ & & 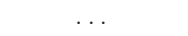 & $\ldots$ & $\ldots$ & 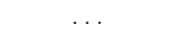 & & .. & \\
\hline 553 & & 11.8 & 2741. & $14.570 \pm 0.003$ & $14.058 \pm 0.001$ & $13.526 \pm 0.002$ & $12.961 \pm 0.002$ & $12.706 \pm 0.003$ & $12.368 \pm 0$ & $12.515 \pm 0.038$ & $12.359 \pm 0.025$ & $12.271 \pm$ & $12.598 \pm 0.029$ & $12.358 \pm 0.027$ & . & ( \\
\hline 553 & & $18.32-02$ & 5409. & $14.615 \pm 0.003$ & $14.106 \pm 0.001$ & $13.557 \pm 0.002$ & $12.979 \pm 0.002$ & $12.734 \pm 0.003$ & & $12.519 \pm 0.047$ & $12.500 \pm 0.031$ & & $12.550 \pm 0.025$ & $12.357 \pm 0.026$ & & 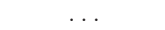 \\
\hline 53 & 39 & $20.23-02$ & 3825. & $1.796 \pm 0.003$ & $14.135 \pm 0.001$ & $13.566 \pm 0.002$ & $13.068 \pm 0.002$ & $12.781 \pm 0.003$ & $12.383 \pm 0.038$ & $12.404 \pm 0.030$ & $12.244 \pm 0.026$ & $12.179 \pm 0.061$ & $12.585 \pm 0.025$ & $12.287 \pm 0.027$ & $11.832 \pm 0.257$ & \\
\hline & & & 2736. & $.670 \pm 0.003$ & $1.109 \pm 0.001$ & $13.572 \pm 0.002$ & $13.036 \pm 0.002$ & $12.730 \pm 0.003$ & $12.390 \pm 0.033$ & $12.424 \pm$ & $12.328 \pm 0.022$ & $12.376 \pm$ & $12.588 \pm$ & $12.370 \pm 0.026$ & & \\
\hline 053 & & 32.30 & 0223. & $14.717 \pm 0.003$ & $14.230 \pm 0.001$ & $13.633 \pm 0.002$ & $13.102 \pm 0.002$ & $12.784 \pm 0.003$ & & $12.134 \pm 0.028$ & & $11.396 \pm 0$ & $12.439 \pm 0.029$ & $12.012 \pm 0.031$ & 4 & . \\
\hline 053 & 38 & 35.8 & 3313. & $14.957 \pm 0.003$ & $14.296 \pm 0.001$ & $13.694 \pm 0.002$ & $13.195 \pm 0.002$ & $12.847 \pm 0.003$ & $12.350 \pm 0.029$ & $12.119 \pm 0.039$ & $11.869 \pm 0.039$ & $11.319 \pm 0.039$ & & & & \\
\hline & & & 0220. & $2 \pm$ & $613 \pm 0$ & $54+8,8$ & $3.273 \pm 0$ & $12.756 \pm$ & & $11.585=$ & & $71-$ & r & 1 & $66=$ & \\
\hline 053 & & 11.8 & 4556. & $15.116 \pm 0.004$ & $4.478 \pm 0.001$ & $13.880 \pm 0.002$ & $13.328 \pm 0.003$ & $13.013 \pm 0.003$ & $12.699 \pm 0$ & $12.728 \pm 0$ & $12.625 \pm$ & $12.523 \pm 0$ & 027 & 12.518 & & $\cdots$ \\
\hline 553 & 38 & 06.69 & 0414. & $15.171 \pm 0.004$ & $14.481 \pm 0.001$ & $13.892 \pm 0.002$ & $13.388 \pm 0.003$ & $13.061 \pm 0.004$ & $000+$ & & & $\ldots$ & $12.907 \pm 0.026$ & $12.628 \pm 0.028$ & & \\
\hline (5) & & & 5738. & $.172 \pm 0.004$ & $372 \pm 0.001$ & $13.901 \pm 0.002$ & $13.406 \pm 0.003$ & $13.037 \pm 0.004$ & & $\ldots$ & $\ldots$ & $\ldots$ & $12.144 \pm 0.024$ & 0.023 & $10.633=$ & \\
\hline 553 & & & 2445. & $15.418 \pm 0$ & $14.701 \pm 0$ & $14.104 \pm 0$ & $13.572 \pm 0$ & $13.232 \pm 0$ & $12.661 \pm 0.044$ & $12.398 \pm$ & $12.032 \pm$ & $11.330 \pm$ & 27 & $12.284 \pm 0.027$ & 10. & \\
\hline 053 & & 53.9 & 4954. & $15.582 \pm 0.005$ & $15.089 \pm 0.002$ & $14.181 \pm 0.003$ & $12.996 \pm 0.002$ & $12.169 \pm 0.002$ & & & & & $11.190 \pm 0.025$ & $10.54 \Omega 0.022$ & $8.807 \pm 0.029$ & $81 \pm$ \\
\hline 053 & & 12.0 & 1426. & $56 \pm 0.005$ & $.961 \pm 0.002$ & $14.307 \pm 0.003$ & $13.859 \pm 0.003$ & $13.483 \pm 0.005$ & $12.929 \pm 0.048$ & & & & $12.987 \pm 0.026$ & $12.673 \pm 0.028$ & & \\
\hline 5 & & & 4047. & $0 \pm 0$ & $67 \pm 0$ & $1 \pm 0$ & 60 & $4 \pm$ & $13.223 \pm$ & $13.448 \pm 0$ & $13.174 \pm$ & $13.145=$ & & & & \\
\hline 053 & & 15. & 1403. & $15.992 \pm 0.006$ & $203 \pm 0$. & $14.568 \pm 0.003$ & $14.093 \pm 0$. & $13.717 \pm 0$ & $13.246 \pm$ & & & & & & & $\ldots$ \\
\hline 553 & & 51.1 & 2607. & $01 \pm 0.006$ & $448 \pm 0.002$ & $55 \pm 0.004$ & $14.365 \pm 0$. & $14.046 \pm 0$ & $13.643 \pm$ & $13.580 \pm 0.044$ & $13.803 \pm 0.074$ & $13.487=$ & 13 & 13. & & \\
\hline & & & 1214. & $6 \pm 0$ & $26 \pm 0$ & $9 \pm 0$ & $14.345 \pm 0$ & $43 \pm 0$ & $13.034 \pm$ & & & & $1 \pm$ & 12.70 & $10.805 \pm 0.102$ & \\
\hline & 39 & & 3826. & $8 \pm$ & 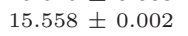 & 4 & 5 & 07 & $13.595 \pm 0.068$ & \pm & $98 \pm$ & $86=$ & & & & \\
\hline 053 & & 58.0 & 3519. & $9 \pm 0.007$ & $661 \pm 0.003$ & $33 \pm 0.004$ & $4.544 \pm 0.005$ & $14.181 \pm 0.007$ & & & & & $13.781 \pm 0.031$ & $13.271 \pm 0.040$ & $11.428 \pm 0.252$ & .. \\
\hline 053 & & 55.4 & & $4 \pm 0$ & $899 \pm 0.003$ & $93 \pm 0$ & $14.479 \pm 0.005$ & $13.890 \pm 0.006$ & $13.008 \pm 0.037$ & $12.663 \pm 0.050$ & $12.156 \pm 0.020$ & $11.286 \pm 0$ & & & & \\
\hline 3 & & & 2715 . & $17 \pm 0$ & $8 \pm 0$ & $3 \pm 0$ & $3 \pm 0$ & $44 \pm 0$ & & & & & 14 & & & \\
\hline 553 & & 53. & 4458. & $988 \pm 0$ & $1 \pm 0$ & $3 \pm 0$ & $31 \pm 0$ & $18 \pm 0$ & $14.095 \pm 0$ & $14.192 \pm$ & $13.900 \pm 0.080$ & $13.921 \pm$ & 31 & 14. & & \\
\hline & & 05. & 2109. & $7 \pm 0$ & $1 \pm 0$ & \pm 0 & $9 \pm 0$ & $23 \pm 0$ & & & & & 14 & 13. & $8 \pm$ & \\
\hline & & & 5351. & $37 \pm 0$ & $16.595 \pm 0$. & $15.551 \pm 0$ & $14.146 \pm 0.004$ & $12.707 \pm 0$ & $10.492 \pm 0.033$ & $9.853 \pm 0.031$ & $9.147 \pm 0$ & $8.442 \pm$ & $10.904 \pm 0.024$ & $9.847 \pm 0.022$ & $8.088 \pm 0.022$ & $5.808 \pm$ \\
\hline & & & 0421. & o & & & & & & & & & & & & \\
\hline 5 & & 34. & & \pm 0 & $0 \pm 0$ & $3 \pm 0$ & $9 \pm 0$ & $14.307 \pm 0$. & & $13.594 \pm$ & & 1 & 8 & 3 & $11.350 \pm 0.154$ & \\
\hline & & & 4033. & $17.518 \pm 0$ & $16.488 \pm 0$. & $15.642 \pm 0$ & $15.067 \pm 0$. & $14.546 \pm 0.009$ & $13.819 \pm$ & $13.520 \pm$ & $13.057 \pm$ & $12.579 \pm$ & 13 & 13. & 工 & \\
\hline 053 & & & 5256. & $.856 \pm 0$ & $16.817 \pm 0.006$ & $16.129 \pm 0.008$ & $15.582 \pm 0.010$ & $15.130 \pm 0.013$ & $14.468 \pm 0.043$ & $14.307 \pm 0.046$ & $14.071 \pm 0.098$ & $13.355 \pm 0$. & $14.819 \pm 0.039$ & $14.266 \pm 0.054$ & & \\
\hline 554 & & 17. & 2654. & $50 \pm$ & $3 \pm 0$ & 88 & 0 & 15 & & $\ldots$ & 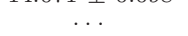 & & & & & \\
\hline & & & 2308. & $2 \pm 0$ & $3 \pm 0$ & 16 & \pm 0 & & & . & .. & . & & & $10.243=$ & \\
\hline & & 53. & 1622. & $18.621 \pm 0.021$ & $17.500 \pm 0.009$ & $16.712 \pm 0.011$ & $16.177 \pm 0.015$ & $15.713 \pm 0.020$ & $15.056 \pm$ & & & & \pm 0 & $14.547 \pm 0.068$ & & \\
\hline & & & & $32 \pm 0.022$ & $.648 \pm 0$. & $45 \pm 0$ & $7 \pm 0.017$ & $15.872 \pm 0.023$ & & & & & \pm & 15.2 & & \\
\hline & & & & \pm & $9 \pm 0$ & \pm & $2 \pm$ & 15 & $15.287 \pm 0.076$ & $15.089 \pm 0.082$ & $14.894 \pm 0.210$ & $14.153 \pm 0$ & & & & \\
\hline & & 10. & $43 \mathrm{c}$ & $5 \pm 0.026$ & $17.916 \pm 0.013$ & $17.021 \pm 0.013$ & $16.416 \pm 0.018$ & $15.921 \pm 0.024$ & & & & & 15 & $6 \pm 0.124$ & $12.023 \pm$ & \\
\hline & & & & $19.043 \pm 0.026$ & $17.972 \pm 0.014$ & $17.082 \pm 0.013$ & $16.520 \pm 0.019$ & $16.017 \pm 0.026$ & 91 & 15 & 426 & $21+2+3$ & $15.597 \pm 0.060$ & $15.606 \pm 0.160$ & & \\
\hline & & & & & & & & & & & & 1 & & & & \\
\hline & & & 37 & $3 \pm 0$ & $75 \pm 0$. & 17 & $0 \pm 0$ & 16 & 15 & 15 & $27 \pm 0$ & $\geq$ & & & & \\
\hline & & & & $19.716 \pm 0.036$ & $18.428 \pm 0.020$ & $17.489 \pm 0.016$ & $16.902 \pm 0.025$ & $16.346 \pm 0.033$ & $15.724 \pm 0$. & $15.463 \pm 0.155$ & $15.464 \pm 0$. & - & & & & \\
\hline 53 & & $25.11-02$ & & $20.114 \pm 0.045$ & $18.836 \pm 0.028$ & $17.821 \pm 0.019$ & $17.211 \pm 0.032$ & $16.675 \pm 0.044$ & $15.893 \pm 0.098$ & $16.019 \pm 0.125$ & $15.699 \pm 0.471$ & $>15.2$ & $16.331 \pm 0.094$ & & & \\
\hline
\end{tabular}


Table 6-Continued

\begin{tabular}{|c|c|c|c|c|c|c|c|c|c|c|c|c|}
\hline $\begin{array}{c}\alpha \\
\mathrm{h}\end{array}$ & $\begin{array}{l}(\mathrm{J} 2000) \\
\mathrm{s}) \quad\left({ }^{\circ}\right.\end{array}$ & $\delta$, & $\begin{array}{c}Z \\
(\mathrm{mag})\end{array}$ & $\begin{array}{c}Y \\
(\mathrm{mag})\end{array}$ & $\begin{array}{c}J \\
(\mathrm{mag})\end{array}$ & $\begin{array}{c}H \\
(\mathrm{mag})\end{array}$ & $\begin{array}{c}K_{s} \\
(\mathrm{mag})\end{array}$ & $\begin{array}{l}{[3.6]} \\
(\mathrm{mag})\end{array}$ & $\begin{array}{l}{[4.5]} \\
(\mathrm{mag})\end{array}$ & $\begin{array}{c}{[5.8]} \\
(\mathrm{mag})\end{array}$ & $\begin{array}{l}{[8.0]} \\
(\mathrm{mag})\end{array}$ & $\begin{array}{c}W 13.4 \mu \mathrm{m} \\
\quad(\mathrm{mag})\end{array}$ \\
\hline 0540 & $06.95-02$ & 23605 & $20.367 \pm 0.051$ & $18.972 \pm 0.031$ & $17.958 \pm 0.021$ & $17.261 \pm 0.034$ & $16.691 \pm 0.044$ & $16.049 \pm 0.107$ & $16.088 \pm 0.141$ & $15.869 \pm 0.515$ & $\geq 15.2$ & . \\
\hline $0538:$ & $33.30-02$ & 2100 & $20.052 \pm 0.043$ & $18.943 \pm 0.031$ & $17.991 \pm 0.021$ & $17.417 \pm 0.038$ & $16.756 \pm 0.047$ & $16.015 \pm 0.153$ & $15.965 \pm 0.164$ & $15.261 \pm 0.323$ & $14.450 \pm 0.522$ & $\cdots$ \\
\hline 0539 & $57.37-02$ & 25005 & $21.190 \pm 0.082$ & $19.869 \pm 0.069$ & $18.862 \pm 0.036$ & $18.303 \pm 0.081$ & $17.549 \pm 0.093$ & $16.752 \pm 0.130$ & $16.962 \pm 0.226$ & $\geq 16.1$ & $\geq 15.2$ & $\ldots$ \\
\hline 0540 & $07.74-02$ & 2234 & $21.745 \pm 0.116$ & $20.108 \pm 0.086$ & $19.122 \pm 0.043$ & $18.466 \pm 0.094$ & $17.776 \pm 0.114$ & $16.892 \pm 0.152$ & $16.408 \pm 0.159$ & $\sum 16.1$ & $\sum 15.2$ & $\cdots$ \\
\hline 0538 & $44.56-02$ & 25513 & $21.997 \pm 0.137$ & $20.344 \pm 0.106$ & $19.230 \pm 0.047$ & $18.477 \pm 0.094$ & $17.818 \pm 0.118$ & $17.059 \pm 0.156$ & $16.928 \pm 0.212$ & $\geq 16.1$ & $\geq 15.2$ & $\cdots$ \\
\hline 5540 & $08.47-02$ & 24550 & $21.889 \pm 0.127$ & $20.607 \pm 0.134$ & $19.346 \pm 0.051$ & $18.256 \pm 0.078$ & $17.698 \pm 0.106$ & & & & & \\
\hline $0539:$ & $32.42-02$ & 5220 & $21.993 \pm 0.137$ & $20.729 \pm 0.150$ & $19.381 \pm 0.052$ & $18.674 \pm 0.112$ & $17.708 \pm 0.107$ & $16.467 \pm 0.151$ & $16.697 \pm 0.219$ & $\geq 16.1$ & $\geq 15.2$ & $\ldots$ \\
\hline $0538:$ & $39.10-02$ & 2805 & $22.417 \pm 0.184$ & $21.294 \pm 0.100$ & $19.952 \pm 0.079$ & $19.771 \pm 0.300$ & $\geq 18.9$ & & & & & \\
\hline
\end{tabular}

$W 24.6 \mu \mathrm{m} \quad W 312.0 \mu \mathrm{m} \quad W 422.0 \mu \mathrm{m}$ (mag)

(mag)

$\cdots$

Note. - All these 58 sources comply with our $Z J$ criteria for photometric cluster membership. No WISE photometry implies signal-to-noise ratio below 4. 
Table 7. Information about known $\sigma$ Orionis photometric candidates

\begin{tabular}{|c|c|c|c|c|c|c|}
\hline$\left(\begin{array}{ll}\mathrm{h} & \mathrm{r}\end{array}\right.$ & $\begin{array}{cl}\alpha & (\mathrm{J} 2 \\
\mathrm{m} & \mathrm{s}\end{array}$ & $\begin{array}{l}\text { 2000) } \\
\left({ }^{\circ}\right.\end{array}$ & $\begin{array}{l}\delta, \quad, \\
,\end{array}$ & $\begin{array}{l}\text { Infrared excess }{ }^{\mathrm{a}} \\
\qquad(\mu \mathrm{m})\end{array}$ & Other names & References \\
\hline 0539 & 3955.91 & -025 & 5121. & $\mathrm{Y}(4.5)$ & [SWW2004]J053956.023-025123.05 & 22,36 \\
\hline 0540 & 1041.45 & -02 & 2907. & $\mathrm{OL}$ & Mayrit 1797077 & 36 \\
\hline 0539 & 3925.61 & -023 & 3404. & $\mathrm{~N}$ & [SWW2004]J053925.608-023404.04,[HHM2007]1043 & 22,31 \\
\hline 0538 & 828.43 & -03 & 0025. & $\mathrm{~N}$ & [SWW2004]J053828.433-030026.12 & 22 \\
\hline 0537 & 3700.30 & -02 & 2826. & $\mathrm{OL}, \mathrm{Y}(12.0)$ & [SWW 2004]J053700.310-022826.34 & 22 \\
\hline 0536 & 3649.28 & -02 & 4354. & $\mathrm{OL}, \mathrm{Y}(12.0)$ & [SWW 2004]J053649.294-024354.28 & 22 \\
\hline 0537 & 3735.14 & 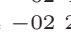 & 2657. & $\mathrm{~N}$ & [HHM2007]107 & 31 \\
\hline 0539 & 3913.47 & -023 & 3739. & $\mathrm{~N}$ & [HHM2007]940 & 31 \\
\hline 0538 & 3847.50 & -02 & 3038. & $\mathrm{~N}$ & S Ori J053847.5-023038 & 42 \\
\hline 0538 & 8852.53 & -02 & 1221. & OL & [SWW2004]J053852.511-021221.41 & 22 \\
\hline 0537 & 3711.68 & -02 & 3156. & $\mathrm{OL}$ & [SWW2004]J053711.681-023156.42 & 22 \\
\hline 0539 & 3911.83 & -02 & 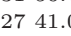 & $\mathrm{N}$ & S Ori 1,[HHM2007]931 & 5,31 \\
\hline 0537 & 3718.32 & -02 & 5409 & $\mathrm{~N}, \mathrm{OL}(3.6)$ & [SWW2004]J053718.330-025409.26 & 22 \\
\hline 0539 & 3920.23 & -02 & 3825. & $\mathrm{~N}$ & S Ori $5,[$ HHM2007]999 & 5,31 \\
\hline 0537 & 3755.12 & -02 & 2736. & $\mathrm{~N}$ & [SWW2004]J053755.113-022736.11, [HHM2007]251 & 22,31 \\
\hline 0538 & 3832.30 & $-03 c$ & 0223. & $\mathrm{Y}(4.5,12.0), \mathrm{OL}(3.6)$ & [SWW2004]J053832.291-030223.57 & 22 \\
\hline 0538 & 8835.83 & -02 & 3313. & $\mathrm{Y}(4.5,8.0)$ & [HHM2007]614 & 26,31 \\
\hline 0538 & 3838.59 & -03 & 0220. & $\mathrm{Y}(4.5,12.0), \mathrm{OL}(3.6)$ & Mayrit 1583183 & 36 \\
\hline 0538 & 8811.89 & -02 & 4556.8 & N & S Ori J053811.9-024557 & 20 \\
\hline 0538 & 3806.69 & -03 & 0414. & OL & Mayrit 1788199 & 36 \\
\hline 0540 & 1006.79 & -025 & 5738.8 & $\mathrm{OL}, \mathrm{Y}(12.0)$ & [SWW 2004]J054006.707-025738.98 & 22 \\
\hline 0539 & 3944.50 & -02 & 2445. & $\mathrm{Y}(4.5,8.0,12.0)$ & S Ori J053944.5-022445 & 42 \\
\hline 0537 & 3753.98 & -02 & 4954. & $\mathrm{OL}, \mathrm{Y}(12.0)$ & [WB2004]10 & 22,23 \\
\hline 0539 & 9912.04 & -02 & 1426. & $\mathrm{OL}$ & {$[\mathrm{SE2} 2004] 46$} & 19 \\
\hline 0539 & 3915.10 & -02 & 4047. & $\mathrm{~N}$ & S Ori 16, [HнM2007] 957 & 5,31 \\
\hline 0539 & 3915.96 & -02 & 1403. & $\mathrm{OL}$ & {$[\mathrm{LO} 2009] 37$} & 39 \\
\hline 0537 & 3751.11 & -02 & 2607. & $\mathrm{~N}$ & S Ori 23 & 5,31 \\
\hline 0539 & 3907.56 & -02 & 1214. & $\mathrm{OL}, \mathrm{Y}(12.0)$ & [SE2004]43 & 19 \\
\hline 0539 & 3915.76 & -02 & 3826. & $\mathrm{~N}$ & S Ori J053915.8-023826 & 42 \\
\hline 0536 & 3658.07 & -02 & 3519. & $\mathrm{OL}, \mathrm{Y}(12.0)$ & S Ori 33 & 5 \\
\hline 0538 & 3855.42 & -02 & 4120. & $\mathrm{Y}(4.5,8.0)$ & S Ori J053855.4-024121 & 32 \\
\hline 0537 & 3707.61 & -02 & 2715. & $\mathrm{OL}, \mathrm{Y}(12.0)$ & S Ori J053707.6-022715 & 42 \\
\hline 0538 & 8853.82 & -02 & 4458.8 & $\mathrm{Y}(12.0)$ & S Ori J053853.8-024459 & 20 \\
\hline 0537 & 3705.17 & -02 & 2109. & $\mathrm{OL}, \mathrm{Y}(12.0)$ & Mayrit 1738301 & 36 \\
\hline 0538 & 8834.46 & -025 & 5351. & $\mathrm{Y}(4.5,8.0,12.0)$ & Mayrit 1082188 & 36 \\
\hline 0538 & 3827.97 & -03 & 0421. & OL & Mayrit 1720188 & 36 \\
\hline 0537 & 3734.57 & -025 & 5558. & $\mathrm{Y}(4.5,12.0), \mathrm{OL}(3.6)$ & S Ori J053734.5-025559 & 30 \\
\hline 0538 & 3854.92 & -02 & 4033. & $\mathrm{Y}(4.5,8.0)$ & S Ori J053855.0-024034,[HнM2007]798 & 30 \\
\hline 0539 & 3918.13 & -025 & 5256. & $\mathrm{Y}(4.5,8.0)$ & S Ori J053918.1-025257 & 21,32 \\
\hline 0540 & 1017.80 & -02 & 2654. & $\mathrm{OL}$ & [LO2009]63 & 39 \\
\hline 0540 & 1018.38 & -02 & 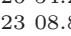 & $\mathrm{OL}, \mathrm{Y}(12.0)$ & [LO2009]64 & 39 \\
\hline 0539 & 3953.06 & -02 & 1622. & $\mathrm{OL}$ & {$[\mathrm{SE} 2004] 114$} & 19 \\
\hline 0540 & 1020.79 & -02 & 2400. & $\mathrm{OL}$ & [LO2009]65 & 39 \\
\hline 0539 & 3946.46 & -02 & 2423. & $\mathrm{Y}(4.5,8.0)$ & S Ori J053946.5-022423 & 9 \\
\hline 0537 & 3710.00 & -02 & $\begin{array}{lll}43 & 02 . \\
\end{array}$ & $\mathrm{OL}, \mathrm{Y}(12.0)$ & S Ori J053710.0-024302 & 8 \\
\hline 0539 & 3929.36 & -02 & 4637. & $\mathrm{~N}$ & S Ori J053929.4-024636 & 21 \\
\hline 0538 & 8814.64 & $=-02$ & 4015. & $\mathrm{~N}$ & S Ori 47 & $5,6,10,11,34,38$ \\
\hline 0539 & 3910.80 & -02 & 3714. & $\mathrm{~N}$ & S Ori 50 & $7,11,34,38$ \\
\hline 0539 & 3913.93 & -02 & 1621. & $\mathrm{Y}(4.5)$ & S Ori J053913.9-021621 & 30 \\
\hline & & & & & & \\
\hline
\end{tabular}




\section{Table 7 - Continued}

\begin{tabular}{|c|c|c|c|c|c|}
\hline$\left(\begin{array}{lll} & \alpha & (\mathrm{J} 2 \\
& \mathrm{m} & \mathrm{s}\end{array}\right)$ & $\begin{array}{l}\text { 1000) } \\
\left({ }^{\circ}\right.\end{array}$ & $\delta, \quad, \quad$ & $\begin{array}{c}\text { Infrared excess }{ }^{\mathrm{a}} \\
\quad(\mu \mathrm{m})\end{array}$ & Other names & References \\
\hline 054006.95 & -023 & 3605.1 & $\mathrm{~N}$ & S Ori J054007.0-023604 & 21 \\
\hline 053833.30 & -022 & 2100.0 & $\mathrm{Y}(4.5,8.0)$ & S Ori 54 & $7,11,33,34,38$ \\
\hline $05 \quad 3957.37$ & -025 & 5005.9 & $\mathrm{~N}$ & S Ori J053957.4-025006 & 30 \\
\hline $05 \quad 40 \quad 07.74$ & -022 & 2234.4 & $\mathrm{~N}$ & S Ori J054007.8-022234 & 30 \\
\hline 053844.56 & -025 & 5513.6 & $\mathrm{~N}$ & S Ori J053844.5-025512 & 30 \\
\hline $\begin{array}{lll}05 & 40 & 08.47\end{array}$ & -024 & 4550.7 & $\mathrm{OL}$ & S Ori J054008.5-024551 & 30 \\
\hline 053932.42 & -025 & 5220.0 & $\mathrm{~N}$ & S Ori J053932.4-025220 & 30 \\
\hline 053839.10 & -022 & 2805.0 & OL & S Ori 68 & $7,10,11,34,38$ \\
\hline
\end{tabular}

Note. - All these 58 sources comply with our $Z J$ criteria for photometric cluster membership.

"Infrared flux excesses ("Y") based on Spitzer (4.5 and $8.0 \mu \mathrm{m})$ and WISE $(12.0 \mu \mathrm{m})$ (hes are denoted with "N". Objects off limits of the Spitzer images are indicated with "OL"

References. - (1) Haro \& Moreno (1953); (2) Wiramihardja et al. (1991); (3) Wolk (1996); (4) Reipurth et al. (1998); (5) Béjar et al. (1999); (6) Zapatero Osorio et al. (1999); (7) Zapatero Osorio et al. (2000); (8) Béjar (2001); (9) Béjar et al. (2001); (10) Martín et al. (2001); (11) Barrado y Navascués et al. (2001); (12) Zapatero Osorio et al. (2002b); (13) Barrado y Navascués et al. (2002b); (14) Zapatero Osorio et al. (2002a); (15) Barrado y Navascués et al. (2003); (16) Jayawardhana et al. (2003); (17) McGovern et al. (2004); (18) Andrews et al. (2004); (19) Scholz \& Eislöffel (2004); (20) Béjar et al. (2004b); (21) Caballero et al. (2004); (22) Sherry et al. (2004); (23) Weaver \& Babcock (2004); (24) Kenyon et al. (2005); (25) Burningham et al. (2005); (26) Caballero (2006); (27) Franciosini et al. (2006); (28) Oliveira et al. (2006); (29) Caballero et al. (2006); (30) Gonzalez-García et al. (2006); (31) Hernandez et al. (2007); (32) Caballero et al. (2007); (33) Zapatero Osorio et al. ballero (2008c); (37) Sacco et al. (2008); (38) Luhman et al. (2008); (39) L et al. (2009); (40) Bihain et al. (2009); (41) Peña Ramírez et al. (2011); (42) Bújar

et al. $(2011)$ 
Table 8. Near and mid infrared photometry of new $\sigma$ Orionis photometric candidates

\begin{tabular}{|c|c|c|c|c|c|c|c|c|c|c|c|c|c|c|c|}
\hline (l $\mathrm{h}$ m & $\begin{array}{ll}\alpha & (\mathrm{J} 200 \\
n^{2} & \mathrm{~s}) \\
\end{array}$ & & $\delta$ & ") & $\begin{array}{c}Z \\
\text { (mag) }\end{array}$ & $\begin{array}{c}Y \\
(\mathrm{mag})\end{array}$ & $\begin{array}{c}J \\
(\mathrm{mag})\end{array}$ & $\begin{array}{c}H \\
(\mathrm{mag})\end{array}$ & $\begin{array}{c}K_{s} \\
(\mathrm{mag})\end{array}$ & $\begin{array}{c}{[3.6]} \\
(\mathrm{mag})\end{array}$ & $\begin{array}{c}{[4.5]} \\
(\mathrm{mag})\end{array}$ & $\begin{array}{c}{[5.8]} \\
(\mathrm{mag})\end{array}$ & $\begin{array}{c}{[8.0]} \\
(\mathrm{mag})\end{array}$ & Name & Infrarec \\
\hline 0538 & 26.50 & -02 & 09 & 25.7 & $20.463 \pm 0.054$ & $1 \pm 0.039$ & $18.252 \pm 0.025$ & $17.663 \pm 0.047$ & $17.062 \pm 0.061$ & $16.220 \pm 0.102$ & $\ldots$ & $>16.1$ & $\ldots$ & S Ori J053826.5-020926 & $\mathrm{OL}$ \\
\hline 0538 & 29.62 & -03 & & 38.2 & $20.585 \pm 0.058$ & $19.416 \pm 0.048$ & $18.404 \pm 0.027$ & $17.731 \pm 0.050$ & $17.049 \pm 0.060$ & $\ldots$ & & $\ldots$ & $\ldots$ & S Ori J053829.6-030438 & $\mathrm{OL}$ \\
\hline 0538 & 29.51 & -02 & 59 & $59.1^{\mathrm{b}}$ & $20.874 \pm 0.068$ & $19.513 \pm 0.050$ & $18.416 \pm 0.027$ & $17.662 \pm 0.047$ & $17.093 \pm 0.063$ & & $16.332 \pm 0.146$ & & $\geq 15.2$ & S Ori J053829.5-025959 & $\mathrm{Y}$ \\
\hline 0538 & 24.71 & -03 & 00 & 28.3 & $21.051 \pm 0.075$ & $19.728 \pm 0.061$ & $18.643 \pm 0.032$ & $18.089 \pm 0.067$ & $17.386 \pm 0.081$ & & $16.666 \pm 0.171$ & & $\geq 15.2$ & S Ori J053824.7-030028 & $\mathrm{N}$ \\
\hline 0538 & 29.52 & -02 & 29 & 37.0 & $21.330 \pm 0.089$ & $20.035 \pm 0.080$ & $18.801 \pm 0.035$ & $18.068 \pm 0.066$ & $17.461 \pm 0.086$ & $16.385 \pm 0.119$ & $16.158 \pm 0.134$ & $\geq 16.1$ & $\geq 15.2$ & S Ori J053829.5-022937 & $\mathrm{N}$ \\
\hline 0538 & 57.51 & -02 & 29 & 05.5 & $21.947 \pm 0.132$ & $20.216 \pm 0.095$ & $19.043 \pm 0.041$ & $18.403 \pm 0.089$ & $17.517 \pm 0.090$ & $16.580 \pm 0.118$ & $16.578 \pm 0.165$ & $\geq 16.1$ & $\geq 15.2$ & S Ori J053857.5-022905 & $\mathrm{N}$ \\
\hline 0537 & 05.49 & -02 & 51 & 29.0 & $21.659 \pm 0.109$ & $20.136 \pm 0.088$ & $19.108 \pm 0.043$ & $18.471 \pm 0.094$ & $17.844 \pm 0.121$ & & & & & S Ori J053705.5-025129 & $\mathrm{OL}$ \\
\hline 0538 & 03.23 & -02 & 26 & 56.8 & $21.821 \pm 0.122$ & $20.435 \pm 0.115$ & $19.259 \pm 0.048$ & $18.591 \pm 0.104$ & $17.877 \pm 0.124$ & $17.139 \pm 0.168$ & $16.908 \pm 0.214$ & $\geq 16.1$ & $\geq 15.2$ & S Ori J053803.2-022657 & \\
\hline 0540 & 00.04 & -02 & 40 & 33.1 & $21.726 \pm 0.114$ & $20.654 \pm 0.140$ & $19.307 \pm 0.049$ & $18.568 \pm 0.102$ & $17.764 \pm 0.112$ & $16.945 \pm 0.167$ & $16.654 \pm 0.171$ & $\geq 16.1$ & $\geq 15.2$ & S Ori J054000.0-024033 & $\mathrm{Y}$ \\
\hline 0538 & 04.65 & -02 & 13 & $52.5^{\mathrm{c}}$ & $23.105 \pm 0.300$ & $20.213 \pm 0.094$ & $19.332 \pm 0.050$ & $19.241 \pm 0.186$ & $\geq 18.9$ & $18.183 \pm 0.354$ & & $\geq 16.1$ & & S Ori J053804.6-021352 & $\mathrm{OL}$ \\
\hline 0538 & 00.06 & -02 & 47 & 06.6 & $22.433 \pm 0.187$ & $20.549 \pm 0.128$ & $19.447 \pm 0.054$ & $18.682 \pm 0.113$ & $17.978 \pm 0.136$ & $17.077 \pm 0.164$ & $17.239 \pm 0.298$ & $\geq 16.1$ & $\geq 15.2$ & S Ori J053800.1-024707 & $\mathrm{N}$ \\
\hline 0540 & 37.82 & -02 & 40 & 01.1 & $22.284 \pm 0.167$ & $20.571 \pm 0.130$ & $19.528 \pm 0.057$ & $18.861 \pm 0.133$ & $18.342 \pm 0.188$ & & & & & S Ori J054037.8-024001 & $\mathrm{OL}$ \\
\hline 0540 & 08.06 & -02 & 20 & 03.3 & $22.289 \pm 0.168$ & $21.102 \pm 0.211$ & $19.713 \pm 0.066$ & $18.845 \pm 0.131$ & $18.435 \pm 0.205$ & $17.212 \pm 0.220$ & $17.458 \pm 0.352$ & $\geq 16.1$ & $\geq 15.2$ & S Ori J054008.1-022003 & $\mathrm{N}$ \\
\hline 0540 & 04.48 & -02 & 53 & 31.9 & $22.197 \pm 0.157$ & $20.816 \pm 0.163$ & $19.782 \pm 0.069$ & $18.920 \pm 0.140$ & $17.973 \pm 0.135$ & & $19.400+0.0$ & 210.1 & & S Ori J054004.5-025332 & OI \\
\hline 0540 & 17.34 & -02 & 36 & $22.6^{\mathrm{d}}$ & $22.918 \pm 0.498$ & $21.755 \pm 0.383$ & $19.876 \pm 0.074$ & $19.071 \pm 0.160$ & $18.405 \pm 0.199$ & & & & & S Ori J054017.3-023623 & OL \\
\hline 0538 & 25.29 & -02 & 42 & 25.3 & $22.442 \pm 0.187$ & $\geq 21.6$ & $19.959 \pm 0.079$ & $18.982 \pm 0.148$ & $18.049 \pm 0.145$ & $16.186 \pm 0.120$ & $15.848 \pm 0.123$ & $\geq 16.1$ & $\geq 15.2$ & S Ori J053825.3-024225 & \\
\hline 0538 & 17.20 & -02 & 27 & 22.5 & $22.705 \pm 0.228$ & $21.238 \pm 0.239$ & $19.962 \pm 0.079$ & $18.961 \pm 0.145$ & $18.181 \pm 0.163$ & $17.086 \pm 0.148$ & $16.836 \pm 0.201$ & 16.1 & $\geq 15.2$ & S Ori J053817.2-022722 & $\mathrm{N}$ \\
\hline 0539 & 23.28 & -02 & 12 & $35.0^{\mathrm{d}}$ & $23.010 \pm 0.190$ & $21.111 \pm 0.213$ & $20.009 \pm 0.082$ & $19.474 \pm 0.230$ & $18.226 \pm 0.170$ & $16.561 \pm 0.227$ & $\ldots$ & $\geq 16.1$ & & S Ori J05392 & $\mathrm{OL}$ \\
\hline 0537 & 05.61 & -02 & 25 & 08.0 & $22.955 \pm 0.276$ & $21.318 \pm 0.200$ & $20.107 \pm 0.089$ & $19.205 \pm 0.180$ & $18.274 \pm 0.177$ & & & & & S Ori J053705.6-022508 & $\mathrm{OL}$ \\
\hline 0538 & 48.88 & -02 & 33 & 28.9 & $22.862 \pm 0.257$ & $21.286 \pm 0.250$ & $20.271 \pm 0.101$ & $19.455 \pm 0.226$ & $18.360 \pm 0.191$ & $16.908 \pm 0.237$ & $16.430 \pm 0.223$ & $\geq 16.1$ & $\geq 15.2$ & S Ori J053848.9-023329 & $\mathrm{Y}$ \\
\hline 0537 & 16.83 & -02 & 43 & $07.7^{\mathrm{d}}$ & $23.010 \pm 0.456$ & $21.708 \pm 0.140$ & $20.289 \pm 0.102$ & $19.476 \pm 0.230$ & $18.458 \pm 0.209$ & $16.860 \pm 0.175$ & $4 \pm 0$ & & & $\mathrm{sc}$ & $\mathrm{Y}$ \\
\hline 0537 & 47.27 & -02 & & 15.5 & $22.911 \pm 0.267$ & $\geq 21.6$ & $20.350 \pm 0.107$ & $19.578 \pm 0.252$ & $18.533 \pm 0.224$ & $17.069 \pm 0.182$ & $17.014 \pm 0.246$ & $\geq 16.1$ & $\geq 15.2$ & S Ori J053747.3-022715 & $\mathrm{N}$ \\
\hline 0537 & 37.58 & -02 & 41 & $14.6^{\mathrm{e}}$ & $23.049 \pm 0.297$ & $\geq 21.6$ & $20.418 \pm 0.113$ & $19.250 \pm 0.188$ & $18.641 \pm 0.246$ & $16.632 \pm 0.119$ & $16.403 \pm 0.176$ & $\geq 16.1$ & $\geq 15.2$ & S Ori J053737.6-024115 & $\mathrm{Y}$ \\
\hline
\end{tabular}

Note. - No WISE photometry with few exceptions.

${ }^{a}$ Infrared flux excesses ("Y") based on Spitzer $(4.5 \mu \mathrm{m})$ data. No flux excesses are denoted with "N". Objects off limits of the Spitzer image are indicated with "OL".

${ }^{\mathrm{b}} W 1(3.4 \mu \mathrm{m})=16.800 \pm 0.138 \mathrm{mag}$

${ }^{\mathrm{c}}$ T-type candidate.

${ }^{\mathrm{d}}$ Found in the extended $Z J$ search.

${ }^{\mathrm{e}} W 1(3.4 \mu \mathrm{m})=16.904 \pm 0.149 \mathrm{mag} ; W 2(4.6 \mu \mathrm{m})=16.008 \pm 0.222 \mathrm{mag}$ 
Table 9. Near- and mid-infrared photometry of sources found in the search down to $J$-band completeness

\begin{tabular}{|c|c|c|c|c|c|c|c|c|c|c|c|c|}
\hline$(\mathrm{h}$ & $\begin{array}{c}\alpha \\
\mathrm{m}\end{array}$ & $\begin{array}{ll}\alpha & (\mathrm{J} 20 \\
& \mathrm{s})\end{array}$ & $\begin{array}{r}000) \\
\left(^{\circ}\right.\end{array}$ & $\delta$, & $\left.{ }^{\prime \prime}\right)$ & $\begin{array}{c}Z \\
\text { (mag) }\end{array}$ & $\begin{array}{c}Y \\
(\mathrm{mag})\end{array}$ & $\begin{array}{c}J \\
(\mathrm{mag})\end{array}$ & $\begin{array}{c}H \\
(\mathrm{mag})\end{array}$ & $\begin{array}{c}K_{s} \\
(\mathrm{mag})\end{array}$ & $\begin{array}{c}{[3.6]} \\
(\mathrm{mag})\end{array}$ & $\begin{array}{c}{[4.5]} \\
(\mathrm{mag})\end{array}$ \\
\hline 05 & 39 & 04.51 & -03 & 02 & 13.5 & $\geq 23.1$ & $\geq 21.6$ & $20.320 \pm 0.105$ & $19.542 \pm 0.244$ & $18.642 \pm 0.246$ & . & $17.484 \pm 0.359$ \\
\hline 05 & 37 & 42.60 & -02 & 58 & 40.3 & $\geq 23.1$ & $\geq 21.6$ & $.501 \pm 0.121$ & $9.685 \pm 0$ & $19.132 \pm$ & . & $.725=$ \\
\hline 05 & 39 & 43.51 & -03 & 01 & 56.2 & $\geq 23.1$ & $\geq 21.6$ & $20.502 \pm 0.121$ & $19.333 \pm 0.202$ & $18.846 \pm 0.297$ & $\cdots$ & $16.410 \pm 0.154$ \\
\hline 05 & 38 & 18.20 & -02 & 46 & $36.2^{\mathrm{a}}$ & $\geq 23.1$ & $\geq 21.6$ & $20.559 \pm 0.127$ & $\geq 19.9$ & $18.669 \pm 0.253$ & $17.178 \pm 0.178$ & $16.738 \pm 0.195$ \\
\hline 05 & 40 & 38.42 & -02 & 28 & 06.6 & $\geq 23.1$ & $\geq 21.6$ & $20.599 \pm 0.131$ & $19.249 \pm 0$. & $18.105 \pm 0.152$ & $\cdots$ & $\cdots$ \\
\hline 05 & 38 & 27.60 & -02 & 09 & 09.0 & $\geq 23.1$ & $\geq 21.6$ & $20.635 \pm 0.135$ & $\sim 19.9$ & $\geq 18.9$ & $18.14 \pm 0$ & $\cdots$ \\
\hline 05 & 40 & 24.32 & -02 & 44 & 44.3 & $\geq 23.1$ & $\geq 21.6$ & $20.683 \pm 0.140$ & $\sim 19.9$ & $\geq 18.9$ & & $\cdots$ \\
\hline 05 & 40 & 40.73 & -02 & 38 & 10.8 & $\geq 23.1$ & $\geq 21.6$ & $20.691 \pm 0.141$ & $19.434 \pm 0$ & & $\cdots$ & $\cdots$ \\
\hline 05 & 37 & 55.81 & -02 & 41 & 51.3 & $\geq 23.1$ & $\geq 21.6$ & $20.728 \pm 0.146$ & $\geq 19.9$ & $\geq 18.9$ & $17.246 \pm 0.178$ & $16.698 \pm 0.178$ \\
\hline 05 & 40 & 25.39 & -02 & 42 & 59.7 & $\geq 23.1$ & $\geq 21.6$ & $20.730 \pm 0.146$ & $\geq 1$ & $18.527 \pm 0.222$ & $\cdots$ & $\cdots$ \\
\hline 05 & 37 & 05.51 & -02 & 33 & 58.0 & $\geq 23.1$ & $\geq 21.6$ & $20.740 \pm 0.147$ & $19.773 \pm 0.301$ & $18.766 \pm 0.276$ & $\cdots$ & $\cdots$ \\
\hline 05 & 39 & 43.18 & -02 & 48 & 59.5 & $\geq 23.1$ & $\geq 21.6$ & $20.759 \pm 0.150$ & $19.607 \pm 0$ & $19.255 \pm 0.300$ & $17.263 \pm$ & $56 \pm$ \\
\hline 05 & 38 & 31.67 & -03 & 00 & 36.3 & $\geq 23.1$ & $\geq 21.6$ & $20.761 \pm 0.1$ & $\geq 19.9$ & $\geq 18.9$ & $\cdots$ & $16.966 \pm$ \\
\hline 05 & 40 & 26.44 & -02 & 31 & 00.8 & $\geq 23.1$ & $\geq 21.6$ & $20.772 \pm 0.151$ & $19.875 \pm 0$ & $19.090 \pm 0$. & $\cdots$ & $\cdots$ \\
\hline 05 & 40 & 17.66 & -02 & 27 & 47.7 & $\geq 23.1$ & $\geq 21.6$ & $20.813 \pm 0.156$ & $\geq 19.9$ & $18.874 \pm 0$ & $\cdots$ & $\cdots$ \\
\hline 05 & 39 & 16.38 & -03 & 01 & 11.1 & $\geq 23.1$ & $\geq 21.6$ & $20.839 \pm 0.160$ & $\geq 1$ & & $\cdots$ & $16.693 \pm$ \\
\hline 05 & 40 & 11.62 & -02 & 56 & 39.4 & $\overline{\geq} 23.1$ & $\overline{\geq} 21.6$ & $20.840 \pm 0.160$ & $19.5 \overline{39} \pm 0.200$ & & $\cdots$ & $\cdots$ \\
\hline 05 & 37 & 00.41 & -02 & 42 & 51.1 & $\geq 23.1$ & $\geq 21.6$ & $0.854 \pm 0.162$ & $\geq 19.9$ & & $\cdots$ & $\cdots$ \\
\hline 05 & 38 & 38.78 & -02 & 54 & $21.1^{\mathrm{a}}$ & $\geq 23.1$ & $\geq 21.6$ & $20.856 \pm 0.162$ & $\geq 1$ & & $18.505 \pm 0.459$ & $17.573 \pm$ \\
\hline 05 & 37 & 22.45 & -02 & 24 & 30.2 & $\geq 23.1$ & $\geq 21.6$ & $20.862 \pm 0.1$ & $\geq 1$ & & $\cdots$ & $\ldots$ \\
\hline 05 & 39 & 49.04 & -02 & 21 & 47.6 & $\geq 23.1$ & $\geq 21.6$ & $20.879 \pm 0.165$ & $19.561 \pm 0.248$ & & $17.398 \pm 0$ & $16.714 \pm$ \\
\hline 05 & 39 & 51.32 & -02 & 18 & 53.6 & $\geq 23.1$ & $\geq 21.6$ & $20.890 \pm 0.167$ & $\geq 19.9$ & $\geq 18.9$ & $17.606 \pm 0.238$ & $17.179 \pm 0.251$ \\
\hline 05 & 40 & 36.29 & -02 & 44 & $55.9^{\mathrm{b}}$ & $\geq 23.1$ & $\geq 21.6$ & $20.909 \pm 0.169$ & $\geq 19.9$ & $\geq 18.9$ & $\ldots$ & $\cdots$ \\
\hline 05 & 36 & 58. & -02 & 36 & 48 & & & $7=$ & & 19.469 & . & . \\
\hline 05 & 40 & 15.61 & -02 & 26 & 30.4 & $\geq 23.1$ & $\geq 21.6$ & $20.934 \pm 0$ & $\sim 19.9$ & $\geq 1$ & $\cdots$ & $\cdots$ \\
\hline 05 & 40 & 27.99 & -02 & 51 & 16.7 & $\geq 23.1$ & $\geq 21.6$ & $20.943 \pm 0.174$ & $19.474 \pm 0$ & & $\cdots$ & $\cdots$ \\
\hline 05 & 38 & 57.41 & -02 & 08 & 48.9 & $\geq 23.1$ & $\geq 21.6$ & $20.943 \pm 0.174$ & $19.349 \pm 0$ & & $\cdots$ & $\cdots$ \\
\hline 05 & 37 & 11.77 & -02 & 35 & 45.2 & $\geq 23.1$ & $\geq 21.6$ & $20.944 \pm 0.174$ & $19.780 \pm 0.200$ & & $\cdots$ & $\cdots$ \\
\hline 05 & 39 & 12.64 & -02 & 47 & 44.8 & $\geq 23.1$ & $\geq 21.6$ & $20.976 \pm 0.179$ & $\geq 19.9$ & & $17.629 \pm$ & $17.133 \pm$ \\
\hline 05 & 37 & 58.95 & -02 & 22 & 50.8 & $\geq 23.1$ & $\geq 21.6$ & $20.996 \pm 0.182$ & $\geq 19.9$ & $\geq 18.9$ & $17.712 \pm 0.266$ & $17.361 \pm 0.311$ \\
\hline
\end{tabular}

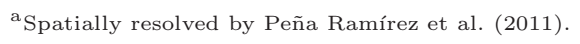

${ }^{\mathrm{b}}$ There is only a $J$-band detection. 
10. Near- and mid-infrared photometry of known $\sigma$ Orionis photometric candidates $(J \geq 16 \mathrm{mag})$ out of the $Z J$ selection criteria

\begin{tabular}{|c|c|c|c|c|c|c|c|c|c|c|c|c|c|c|}
\hline $\begin{array}{c}\alpha \\
\mathrm{m}\end{array}$ & $\begin{array}{l}(\mathrm{J} 2000) \\
\left.{ }^{\mathrm{s}}\right) \quad\left({ }^{\circ}\right.\end{array}$ & & ") & $\begin{array}{c}Z \\
(\mathrm{mag})\end{array}$ & $\begin{array}{c}Y \\
(\mathrm{mag})\end{array}$ & $\begin{array}{c}J \\
(\mathrm{mag})\end{array}$ & $\begin{array}{c}H \\
(\mathrm{mag})\end{array}$ & $\begin{array}{c}K_{s} \\
(\mathrm{mag})\end{array}$ & $\begin{array}{c}{[3.6]} \\
(\mathrm{mag})\end{array}$ & $\begin{array}{c}{[4.5]} \\
(\mathrm{mag})\end{array}$ & $\begin{array}{c}{[5.8]} \\
(\mathrm{mag})\end{array}$ & $\begin{array}{c}{[8.0]} \\
(\mathrm{mag}) \\
\end{array}$ & Other names & References \\
\hline 39 & $47.05-02$ & 25 & 24.5 & $20.298 \pm 0.049$ & $19.679 \pm 0.058$ & $18.937 \pm 0.038$ & $18.363 \pm 0.086$ & $17.988 \pm 0.137$ & & $17.280 \pm 0.380$ & $\geq 16.1$ & $\geq 15.2$ & S Ori 57 & 1,2 \\
\hline 36 & $53.30-02$ & & 12.9 & $21.963 \pm 0.134$ & $20.974 \pm 0.188$ & $19.721 \pm 0.066$ & $19.101 \pm 0.164$ & $18.758 \pm 0.274$ & $\ldots$ & & & & S Ori 64 & 1 \\
\hline 38 & $12.61-02$ & 21 & 37.5 & $22.130 \pm 0.150$ & $20.908 \pm 0.177$ & $19.904 \pm 0.076$ & $19.505 \pm 0.236$ & $19.049 \pm 0.357$ & $18.214 \pm 0.389$ & $18.677 \pm 0.456$ & $\geq 16.1$ & $\geq 15.2$ & S Ori 67 & $1,3,4,5$ \\
\hline 38 & $59.16-02$ & 35 & 26.1 & $\geq 23.1$ & $\geq 21.6$ & $20.329 \pm 0.105$ & $19.731 \pm 0.290$ & $18.971 \pm 0.332$ & $17.650 \pm 0.110$ & $17.510 \pm 0.290$ & $\geq 16.1$ & $\geq 15.2$ & S Ori 72 & 6 \\
\hline 38 & $14.49-02$ & 45 & 11.8 & $\geq 23.1$ & $\geq 21.6$ & $20.626 \pm 0.134$ & $\geq 19.9$ & $\geq 18.9$ & $17.540 \pm 0.226$ & $17.680 \pm 0.424$ & $\geq 16.1$ & $\geq 15.2$ & S Ori 73 & 6,7 \\
\hline 38 & $44.28-02$ & 40 & 07.8 & $21.7 \overline{75} \pm 0.118$ & $\sum 21.6$ & $20.749 \pm 0.148$ & $\sum 19.9$ & $\geq 18.9$ & $\ldots$ & $\ldots$ & $\sum 16.1$ & $\sum 15.2$ & S Ori 74 & 6 \\
\hline 38 & $52.51-02$ & 28 & 45.3 & $23.149 \pm 0.321$ & $\geq 21.6$ & $\geq 21.6$ & $\geq 19.9$ & $\geq 18.9$ & $\geq 18.8$ & $\geq 18.0$ & $\geq 16.1$ & $\geq 15.2$ & S Ori 61 & 1,3 \\
\hline 39 & $18.05-02$ & 28 & 54.1 & $\geq 23.1$ & $\geq 21.6$ & $\geq 21.6$ & $\geq 19.9$ & $\geq 18.9$ & $18 . \overline{21} 5 \pm 0.401$ & $17.8 \overline{1} 2 \pm 0.485$ & $\geq 16.1$ & $\geq 15.2$ & S Ori 69 & $1,2,4,5$ \\
\hline
\end{tabular}

ote. - None shows mid-infrared flux excesses at $8 \mu \mathrm{m}$ nor was detected by WISE.

eferences. - (1) Zapatero Osorio et al. 2000; (2) Martín et al. 2001; (3) Barrado y Navascués et al. 2001; (4) Scholz \& Jayawardhana 2008; (5) Luhman et al. 2008; (6) Bihain 1. 2009; (7) Peña Ramírez et al. 2011. 
Near- and mid-infrared photometry of sources selected in the Western $30^{\prime}-50^{\prime}$ rectangular region

\begin{tabular}{|c|c|c|c|c|c|c|c|c|c|}
\hline $\begin{array}{ll}000) & \delta \\
\left({ }^{\circ}\right. & \\
\end{array}$ & $\stackrel{\delta}{\prime}, \quad$, & $\begin{array}{c}Z \\
(\mathrm{mag}) \\
\end{array}$ & $\begin{array}{c}Y \\
(\mathrm{mag})\end{array}$ & $\begin{array}{c}J \\
(\mathrm{mag})\end{array}$ & $\begin{array}{c}H \\
(\mathrm{mag})\end{array}$ & $\begin{array}{c}K_{s} \\
(\mathrm{mag})\end{array}$ & $\begin{array}{c}W 13.4 \mu \mathrm{m} \\
(\mathrm{mag})\end{array}$ & $\begin{array}{c}W 24.6 \mu \mathrm{m} \\
(\mathrm{mag})\end{array}$ & $\begin{array}{c}W 312.0 \mu \mathrm{m} \\
\quad(\mathrm{mag})\end{array}$ \\
\hline & 2544.4 & $14.059 \pm 0.002$ & $13.710 \pm 0.001$ & $13.085 \pm 0.002$ & $12.514 \pm 0.002$ & $12.192 \pm 0.002$ & $11.774 \pm 0.025$ & $11.359 \pm$ & $9.867 \pm$ \\
\hline & $28 \quad 16.4$ & $14.218 \pm 0.002$ & $13.808 \pm 0.001$ & $13.227 \pm 0.002$ & $12.666 \pm$ & $12.418 \pm$ & $12.202 \pm 0.025$ & $12.012 \pm 0.025$ & \\
\hline & 1756.0 & $14.408 \pm 0.003$ & $13.852 \pm 0.001$ & $13.301 \pm 0.002$ & $12.767 \pm 0.002$ & $12.524 \pm 0.003$ & $11.918 \pm 0.023$ & $11.716 \pm 0.023$ & \\
\hline & 5802.6 & $14.451 \pm 0.003$ & $13.908 \pm 0.001$ & $13.342 \pm 0.002$ & $12.738 \pm 0.002$ & $12.326 \pm 0.002$ & $11.780 \pm 0.024$ & $11.361 \pm 0.022$ & $10.221 \pm 0.063$ \\
\hline & 5053.9 & $14.584 \pm 0.003$ & $14.048 \pm 0.001$ & $13.492 \pm$ & $12.956 \pm$ & $12.707 \pm$ & $12.410 \pm$ & $12.167 \pm$ & \\
\hline & 2526.1 & $15.022 \pm 0.004$ & $14.270 \pm 0.001$ & $13.719 \pm 0.002$ & $13.049 \pm 0.002$ & $12.708 \pm 0.003$ & $12.428 \pm 0.027$ & $12.008 \pm 0.024$ & $10.466 \pm$ \\
\hline & 5048.0 & $15.845 \pm 0.005$ & $15.009 \pm 0.002$ & $14.303 \pm 0.003$ & $13.827 \pm 0.003$ & $13.483 \pm 0.005$ & $13.225 \pm 0.029$ & $12.925 \pm 0.032$ & \\
\hline & 1702.7 & $16.294 \pm 0.007$ & $15.581 \pm 0.002$ & $14.937 \pm 0.004$ & $14.476 \pm 0.005$ & $14.133 \pm 0.007$ & $13.897 \pm 0.032$ & $13.622 \pm 0.040$ & $\ldots$ \\
\hline & 1148.3 & $17.765 \pm 0.014$ & $16.812 \pm 0.006$ & $16.087 \pm 0.008$ & $15.558 \pm 0.010$ & $15.197 \pm 0.014$ & $15.007 \pm$ & $14.650=$ & $\ldots$ \\
\hline & 4240.7 & $17.710 \pm 0.013$ & $16.823 \pm 0.006$ & $16.106 \pm 0.008$ & $15.575 \pm 0.010$ & $15.203 \pm 0.014$ & $14.870 \pm 0.040$ & $14.551 \pm 0.069$ & \\
\hline & $4625.3^{\mathrm{a}}$ & $19.864 \pm 0.039$ & $18.520 \pm 0.021$ & $17.534 \pm 0.016$ & $16.908 \pm 0.025$ & $16.311 \pm 0.033$ & & & .. \\
\hline & 2824.1 & $20.309 \pm 0.049$ & $19.174 \pm 0.038$ & $18.190 \pm 0.024$ & $17.635 \pm 0.046$ & $17.138 \pm 0.065$ & & & .. \\
\hline 14 & $1441.2^{\mathrm{a}}$ & $20.731 \pm 0.062$ & $19.465 \pm 0.048$ & $18.464 \pm 0.028$ & $17.842 \pm 0.055$ & $17.247 \pm 0.071$ & & & \\
\hline & 2207.2 & $21.060 \pm 0.076$ & $19.675 \pm 0.058$ & $18.730 \pm 0.033$ & $18.069 \pm 0.066$ & $17.387 \pm 0.081$ & & & \\
\hline
\end{tabular}

ric candidates already identified by Béjar et al. (2011)

Tone has WISE $22.0 \mu \mathrm{m}$ detections. 
Table 12. Parametrization of the $\sigma$ Orionis mass spectrum and mass function

\begin{tabular}{|c|c|c|c|}
\hline \multirow[b]{2}{*}{ Mass range } & \multicolumn{2}{|c|}{ Case } & \multirow[b]{2}{*}{ Functional form } \\
\hline & $\mathrm{I}$ & II & \\
\hline $19-0.35 M_{\odot}$ & $\alpha=1.73 \pm 0.16$ & $\alpha=1.69 \pm 0.18$ & Power law \\
\hline $0.35-0.006 M_{\odot}$ & $\alpha=0.55 \pm 0.20$ & $\alpha=0.68 \pm 0.23$ & Power law \\
\hline $19-0.006 M_{\odot}$ & $\begin{array}{l}M_{c}=0.27 \pm 0.07 \\
\sigma=0.57 \pm 0.11\end{array}$ & $\begin{array}{l}M_{c}=0.27 \pm 0.11 \\
\sigma=0.68 \pm 0.19\end{array}$ & Lognormal \\
\hline $1+0.006 M_{\odot}$ & $\begin{array}{l}M_{c}=0.25 \pm 0.07 \\
\sigma=0.47 \pm 0.13\end{array}$ & $\begin{array}{l}M_{c}=0.23 \pm 0.11 \\
\sigma=0.58 \pm 0.24\end{array}$ & "Lognormal + Power law \\
\hline $19-1 M_{\odot}$ & $\alpha=2.20 \pm 0.19$ & $\alpha=2.20 \pm 0.19$ & \\
\hline$\overline{1-0.012 M_{\odot}}$ & $\begin{array}{l}M_{c}=0.25 \pm 0.07 \\
\sigma=0.46 \pm 0.13\end{array}$ & $\begin{array}{l}M_{c}=0.24 \pm 0.11 \\
\sigma=0.54 \pm 0.23\end{array}$ & Lognormal + Power law \\
\hline $19-1 M_{\odot}$ & $\alpha=2.20 \pm 0.19$ & $\alpha=2.20 \pm 0.19$ & \\
\hline
\end{tabular}

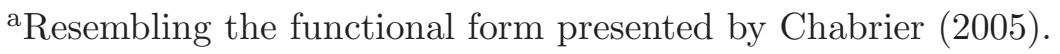

${ }^{\mathrm{b}}$ Resembling the functional form and mass interval used in Chabrier (2005).

Note. - Case I uses masses derived from Siess et al. (2000) for the mass interval 19-1 $M_{\odot}$, and from the Lyon models for the mass interval 1-0.006 $M_{\odot}$. Case II uses Siess et al. (2000) for the interval 19-0.3 $M_{\odot}$, and Lyon models for lower masses. 